\title{
Evaluating the Utility of Permethylated Polysaccharide Solution NMR Data for Characterization of Insoluble Plant Cell Wall Polysaccharides
}

\author{
Ikenna E. Ndukwe, Ian Black, Christian Heiss and Parastoo Azadi* \\ Complex Carbohydrate Research Center, University of Georgia, 315 Riverbend Road, Athens, GA \\ 30602, USA. \\ Corresponding author email: azadi@ccrc.uga.edu
}

\section{Supplemental information}

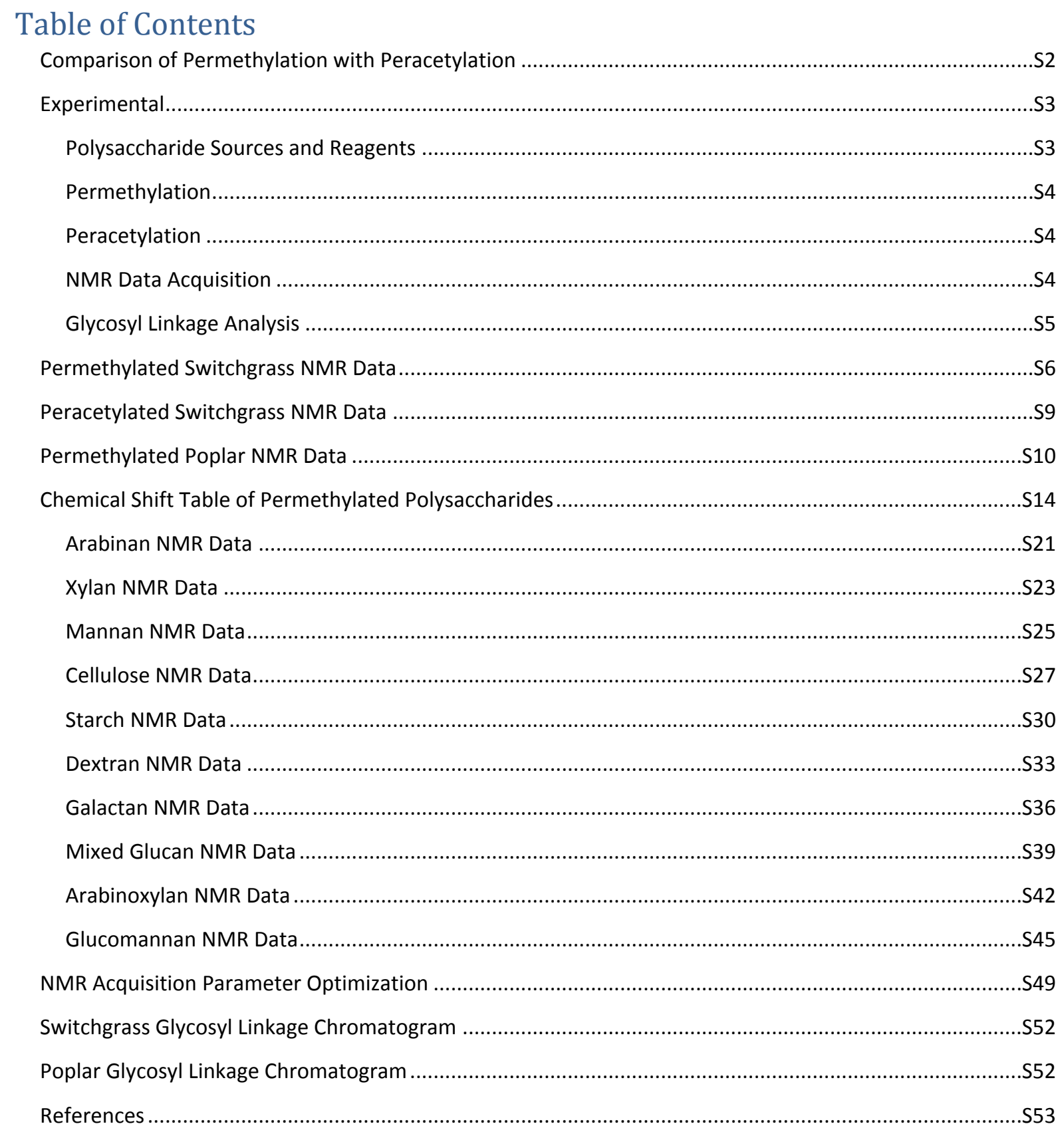




\section{Comparison of Permethylation with Peracetylation}

Two chemical derivatization methods are available to solubilize insoluble polysaccharides, peracetylation and permethylation. We first assessed and compared both of these to determine the most viable approach for polysaccharide structure analysis of plant cell walls. We acquired ${ }^{1} \mathrm{H}$ NMR spectra of derivatized switchgrass samples containing equivalent amounts of material. One of these was peracetylated ${ }^{1}$ and the other permethylated ${ }^{2,3}$ (as discussed here). The results highlight the disparity in sensitivity between both approaches - at least in our hands (Fig. S1). The peracetylated sample required 160 scans to generate a ${ }^{1} \mathrm{H}$ NMR spectrum that has $\sim 50-60 \%$ signal intensity of the ${ }^{1} \mathrm{H}$ NMR spectrum acquired of the permethylated sample, which required only 2 scans. This sensitivity disparity is significant when considering structure analysis of minor plant cell wall components. For instance, the permethylated sample allowed identification of the arabinose residue signal that is $<5 \%$ abundance (from the arabinoxylan present in the sample), whereas this signal was absent or could not be resolved in the peracetylated sample (see sections, 'permethylated switchgrass NMR data' and peracetylated switchgrass NMR data' below). In addition, although a noticeable chemical shift dispersion is observed in the ${ }^{1} \mathrm{H}$ NMR spectrum of the peracetylated sample, this dispersion does not necessarily equate to the desired resolution due to possible coincidental peak overlap of ring protons with the anomeric protons (Fig. S1). Although 2D HSQC should, in principle, provide the necessary resolution to distinguish these peaks, the lack of sensitivity observed in the peracetylated samples limits this approach to the most abundant cell wall components (Fig. S10). For the permethylated switch grass sample, on the other hand, the 2D HSQC spectrum (Fig. S4) provided additional peak resolution of ring residue protons with superior sensitivity that would be necessary to resolve various cell wall polysaccharide components. Due to the unpredictable overlap of acetylated ring protons and anomeric protons, the distinct chemical shift of the anomeric proton in each monomer unit, a unique feature of carbohydrate NMR, helpful for interpretation of underivatized polysaccharide spectra, is lost by acetylation. This is not the case with permethylation, which therefore provides better resolution to tackle the challenging structure analysis of both soluble and insoluble polysaccharide components of complex plant cell wall matrices. 


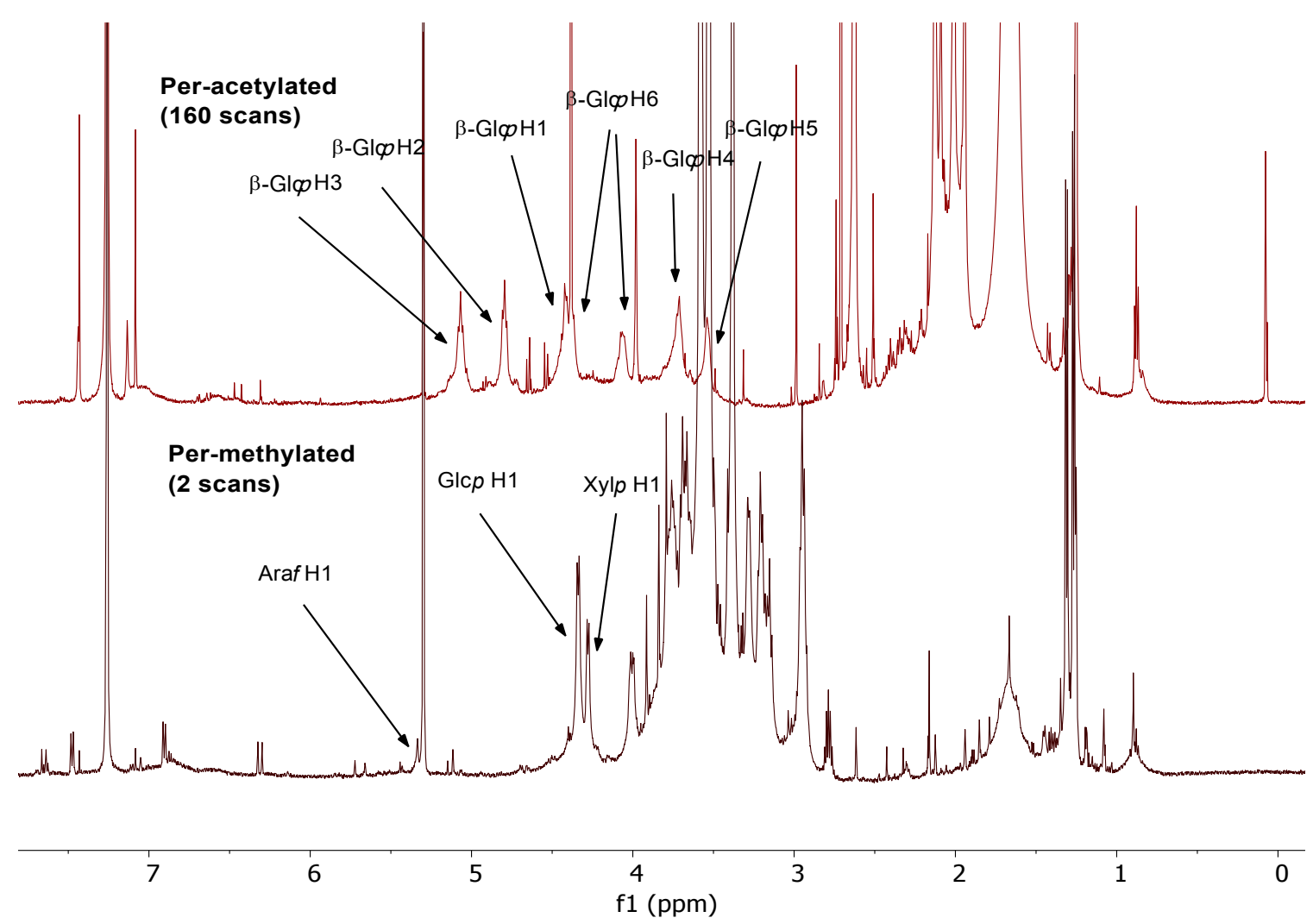

Figure S1: ${ }^{1} \mathrm{H}$ NMR spectra of peracetylated (top) and permethylated (bottom) switchgrass sample. The peracetylated switchgrass ${ }^{1} \mathrm{H}$ NMR spectrum was acquired with 160 scans compared to just 2 scans for the permethylated switchgrass spectrum. The higher sensitivity afforded by permethylation enabled identification of low abundance arabinose residue (from arabinoxylan in the sample) on $\sim 5.8 \mathrm{mg}$ sample (prior to permethylation or peracetylation) on a $600 \mathrm{MHz}$ spectrometer equipped with $5 \mathrm{~mm}$ TXO cryoprobe in a $600 \mu \mathrm{L} \mathrm{CDCl}_{3}$ solution.

\section{Experimental}

\section{Polysaccharide Sources and Reagents}

Ten standard polysaccharides and two plant cell wall materials; namely switchgrass and poplar, were utilized in this study. The polysaccharide standards used were beta glucan (mixed-link glucan, barley), glucomannan (konjac), galactan (potato), linear arabinan (sugar beet), xylan (birchwood), arabinoxylan (wheat), mannan (Ivory nut) - all from Megazyme - and dextran (511 KDa), starch (18727) and cellulose (Avicel PH-101, 11365) - from Sigma-Aldrich. Switchgrass and poplar samples were locally grown and processed. Methyl iodide, dimethyl sulfoxide (DMSO) were also sourced from SigmaAldrich. Molecular sieves were added to the DMSO and left for $24-48 \mathrm{hrs}$ before use to remove associated water molecules that potentially leads to under-methylation. 


\section{Permethylation}

Permethylation of the sample was performed as described by Ciucanu and Kerek ${ }^{3}$ with modifications as introduced by Anumula, K.R., Taylor. ${ }^{2}$ For the analysis, between 5 and $10 \mathrm{mg}$ of sample was used. $500 \mu 1$ of dimethylsulfoxide was added to the standards and ball milled switchgrass and poplar samples. The samples were heated for 5 minutes at $100{ }^{\circ} \mathrm{C}$ in order to help solubilize the samples. To the cooled samples, $500 \mu \mathrm{l}$ sodium hydroxide base ${ }^{2}$ was added and the samples were sonicated for 15 minutes. 150 $\mu l$ iodomethane was then added to the samples and a stir bar was used to mix the samples for 40 minutes. The reaction sequence of base and iodomethane addition was repeated once. After the second round of addition, $2 \mathrm{ml}$ water was added to the samples followed by $2 \mathrm{ml}$ dichloromethane. The samples were then vortexed for 10 seconds to mix and centrifuged to separate the organic/aqueous layers. The top aqueous layer was decanted and $2 \mathrm{ml}$ of fresh water was added to the sample. A total of 5 washes of the sample were performed. After the last wash step, the bottom organic layer was retrieved and placed into a new tube. The dichloromethane was evaporated under nitrogen and the samples were lyophilized to ensure complete removal of water. The dried samples were used for NMR analysis.

\section{Peracetylation}

Acetylation of the ball milled switchgrass sample was performed as described by Pettolino et al. ${ }^{4}$ For comparison, an equivalent amount of sample was used as above for the switchgrass sample. The dried, ball milled sample was dissolved in $100 \mu \mathrm{l}$ dimethylsulfoxide and $50 \mu \mathrm{L}$ 1-methyl imidizole was added. The sample was stirred for 3 hours at room temperature. $25 \mu \mathrm{L}$ acetic anhydride was then added to the sample and it was stirred for 1.5 hours. The solution was then transferred to $30 \mathrm{ml}$ deionized (DI) water and allowed to sit overnight. The sample was then centrifuged at $3000 \mathrm{G}$ for 10 minutes to pellet the sample material. The water was decanted and fresh water was used to wash the sample. After removal of the water from the wash step, the sample was lyophilized. The dried sample was used for NMR analysis.

\section{NMR Data Acquisition}

NMR data utilized in this report were all acquired either a Bruker $600 \mathrm{MHz}$ spectrometer equipped with a $5 \mathrm{~mm}$ TCI cryoprobe or a Varian VNMRS $600 \mathrm{MHz}$ spectrometer equipped with $5 \mathrm{~mm} \mathrm{HNC}$ cryoprobe except otherwise indicated. The permethylated polysaccharide standards were dissolved in $600 \mu \mathrm{L} \mathrm{CDCl}_{3}$ prior to transferring into $5 \mathrm{~mm}$ NMR tubes. NMR data acquired included ${ }^{1} \mathrm{H}$ NMR, COSY or DQFCOSY, HSQC (145 Hz ${ }^{1} J_{\mathrm{CH}}$ delay), HSQC-TOCSY (145 Hz ${ }^{1} J_{\mathrm{CH}}$ delay, $18 \mathrm{~ms}$ mixing time), HMBC ( $8 \mathrm{~Hz}^{\mathrm{n}} J_{\mathrm{CH}}$ delay), TOCSY (80 ms spin-lock time) and NOESY (80 ms mixing time) except otherwise indicated. A recycle delay of $1 \mathrm{~s}$ and $1.5 \mathrm{~s}$ were used, respectively, for 1D and 2D experiments. All datasets where processed of Mestrenova software (version 14.0.1-23559). 


\section{Glycosyl Linkage Analysis}

Glycosyl linkage analysis was performed by combined gas chromatography/mass spectrometry (GC/MS) of the partially methylated alditol acetates (PMAAs) derivatives produced form the samples. The procedure is a slight modification of the one described by Heiss et al. ${ }^{5}$

Briefly, the permethyted samples used for NMR analysis were dried under nitrogen. The samples were then hydrolyzed using $2 \mathrm{M}$ TFA ( $2 \mathrm{~h}$ in sealed tube at $120^{\circ} \mathrm{C}$ ), reduced with $\mathrm{NaBD}$, and acetylated using acetic anhydride/trifloroacetic acid. The resulting PMAAs were analyzed on an Agilent 7890A GC interfaced to a 5975C MSD (mass selective detector, electron impact ionization mode); separation was performed on a $30 \mathrm{~m}$ Supelco SP-2331 bonded phase fused silica capillary column. 


\section{Permethylated Switchgrass NMR Data}

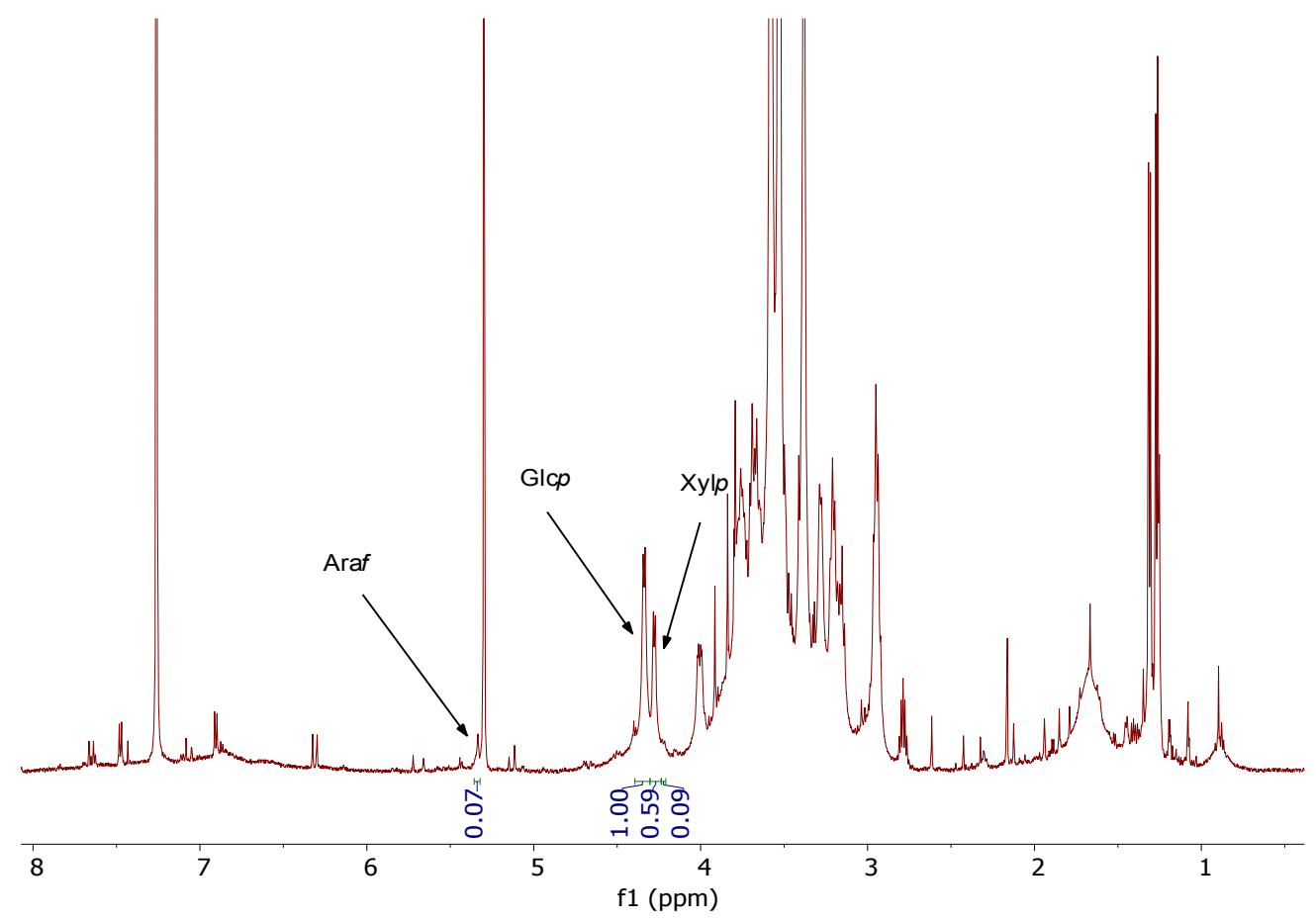

Figure S2: ${ }^{1} \mathrm{H}$ NMR spectrum of permethylated switchgrass sample $(\sim 5.8 \mathrm{mg})$ acquired in $600 \mu \mathrm{L}$ $\mathrm{CDCl}_{3}$ on a Bruker $600 \mathrm{MHz}$ spectrometer quipped with a $5 \mathrm{~mm}$ TXO cryoprobe. Recycle delay $30 \mathrm{~ms}$. Spectrum acquired with 2 scans.

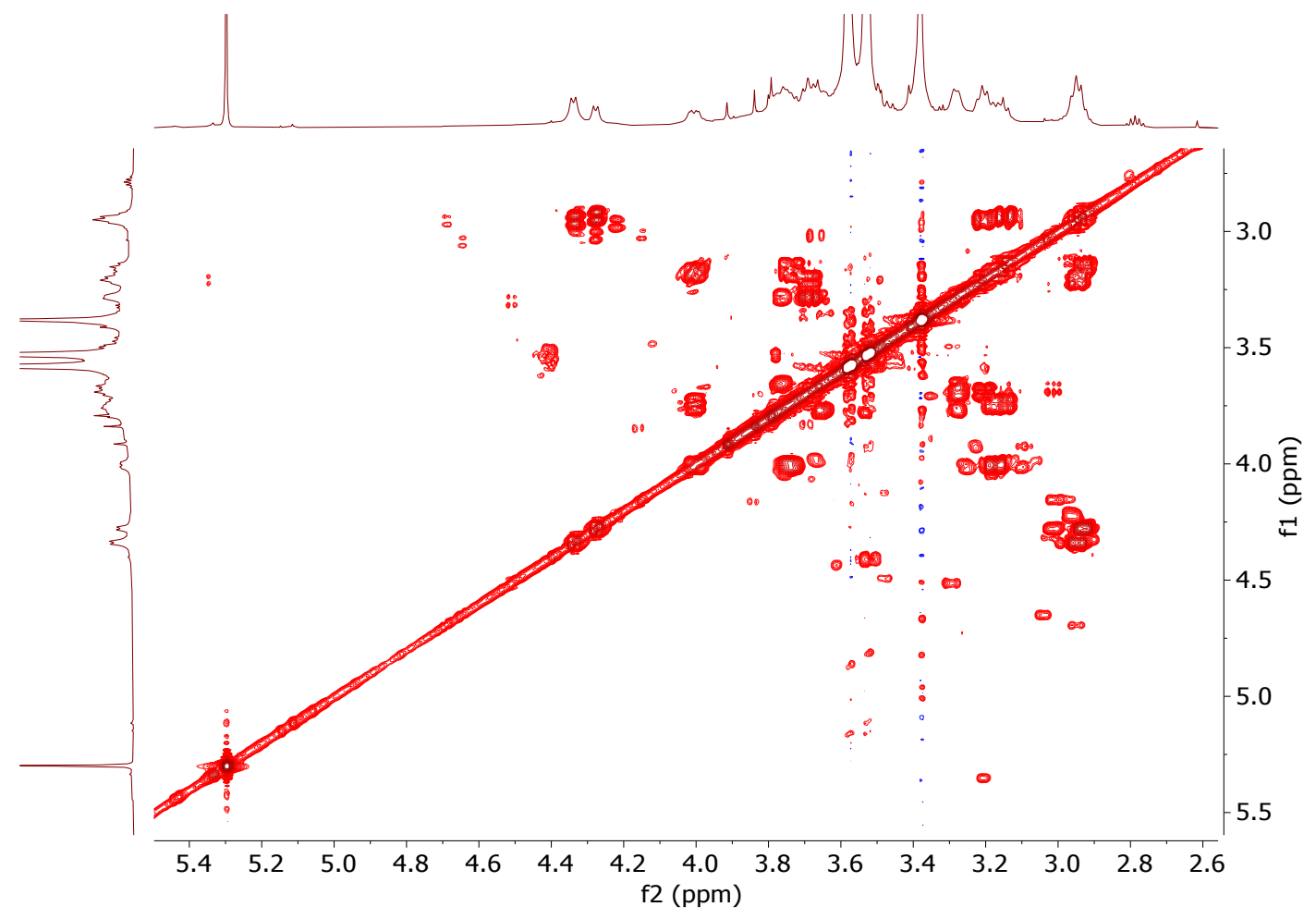

Figure S3: COSY NMR spectrum of permethylated switchgrass sample $(\sim 5.8 \mathrm{mg})$ acquired in $600 \mu \mathrm{L}$ $\mathrm{CDCl}_{3}$ with $1024 * 512(\mathrm{~F} 2 * \mathrm{~F} 1)$ data points on a Bruker $600 \mathrm{MHz}$ spectrometer quipped with a $5 \mathrm{~mm}$ TXO cryoprobe. Spectrum acquired with 4 scans per increment. 


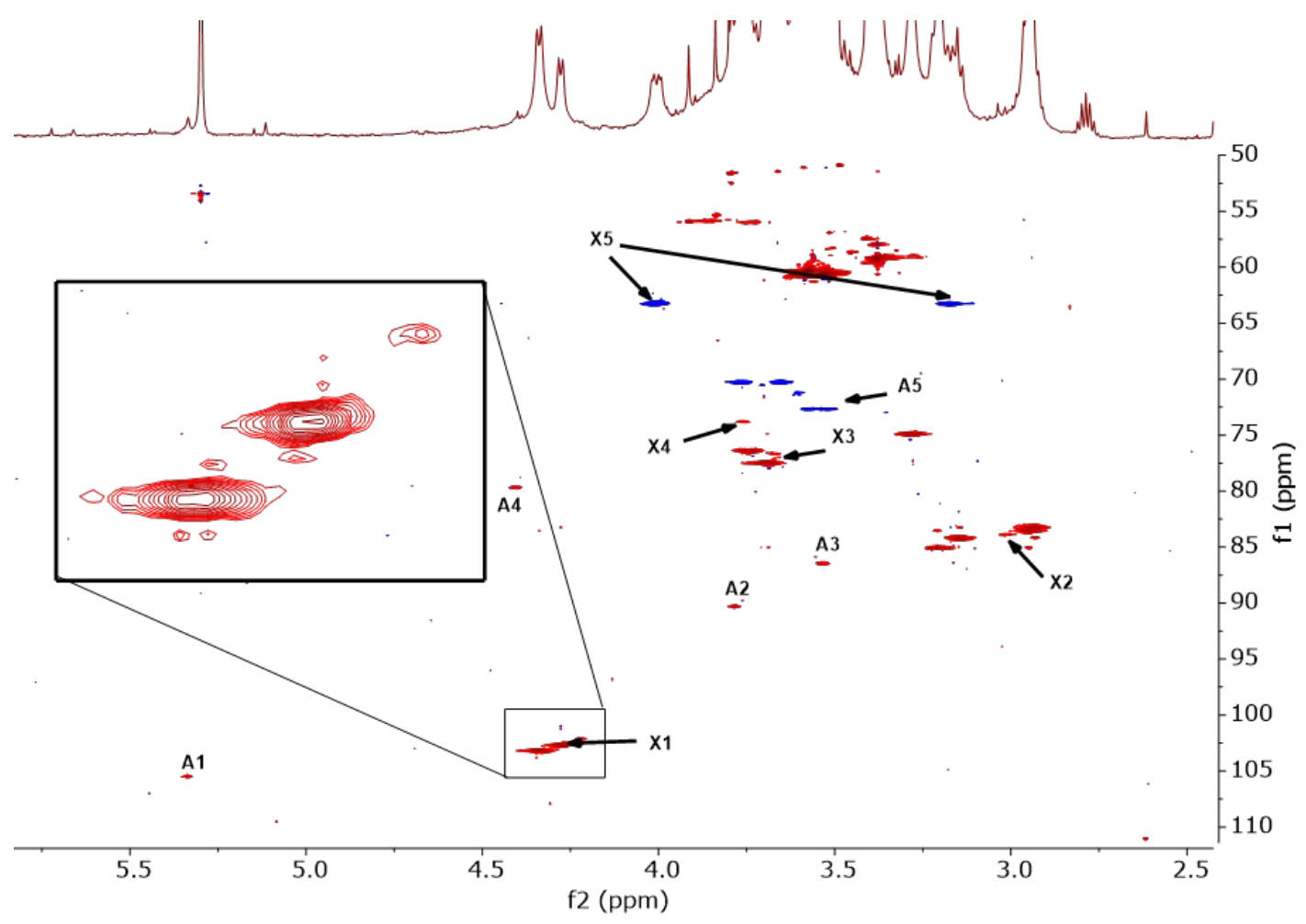

Figure S4: HSQC NMR spectrum of permethylated switchgrass sample $(\sim 5.8 \mathrm{mg})$ acquired in $600 \mu \mathrm{L}$ $\mathrm{CDCl}_{3}$ with $922 * 768(\mathrm{~F} 2 * \mathrm{~F} 1)$ data points on a Bruker $600 \mathrm{MHz}$ spectrometer quipped with a $5 \mathrm{~mm}$ TXO cryoprobe. The residue peak dispersion and sensitivity were sufficient to identity the 3,4-linked xylan residue (annotated as X) and the attached terminal arabinose residue (annotated as A), which is < $5 \%$ signal intensity. Spectrum acquired with 8 scans per increment.

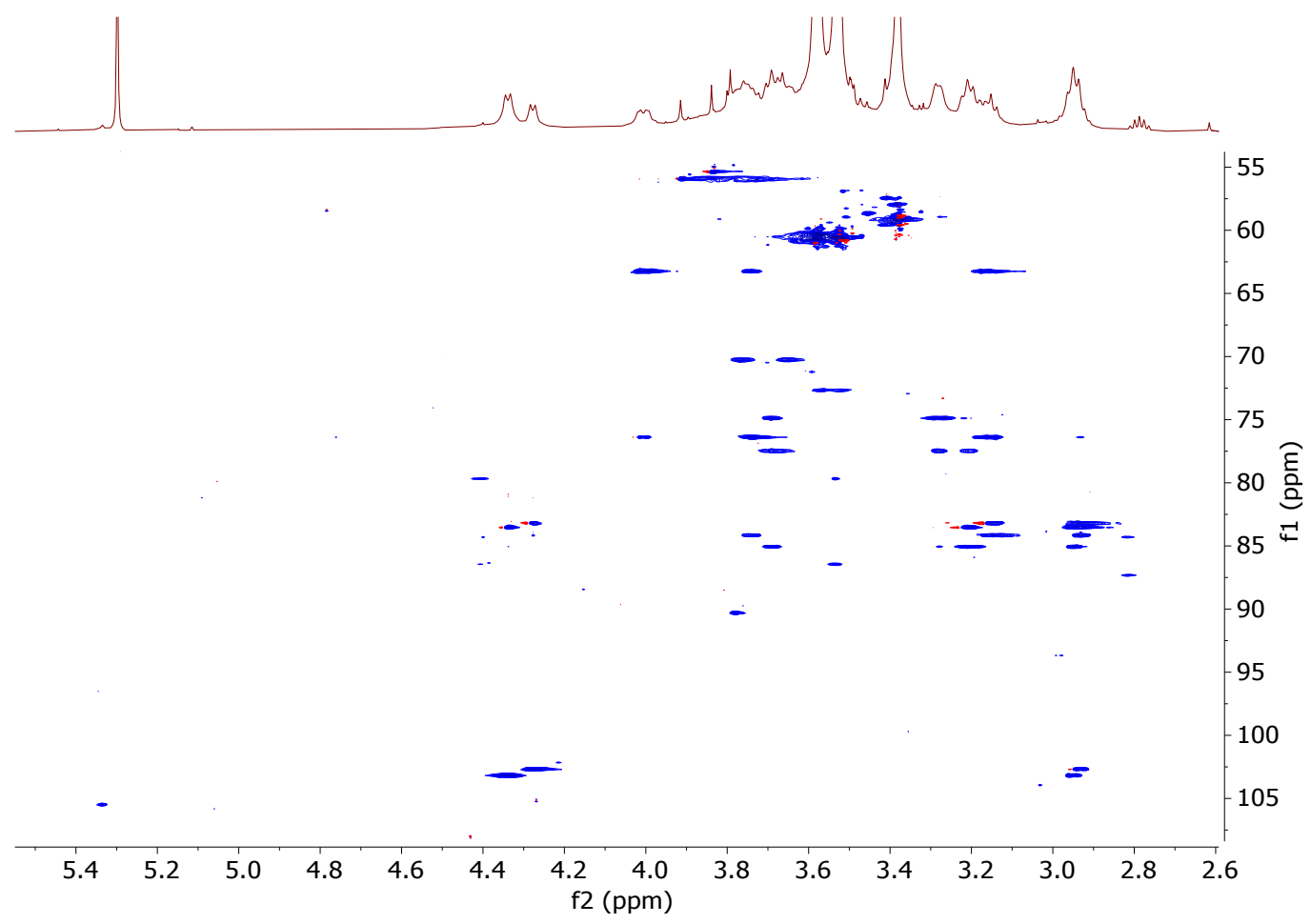

Figure S5: HSQC NMR spectrum of permethylated switchgrass sample $(\sim 5.8 \mathrm{mg})$ acquired in $600 \mu \mathrm{L}$ $\mathrm{CDCl}_{3}$ with $922 * 768(\mathrm{~F} 2 * \mathrm{~F} 1)$ data points on a Bruker $600 \mathrm{MHz}$ spectrometer quipped with a $5 \mathrm{~mm}$ TXO cryoprobe. Spectrum acquired with 16 scans per increment. 


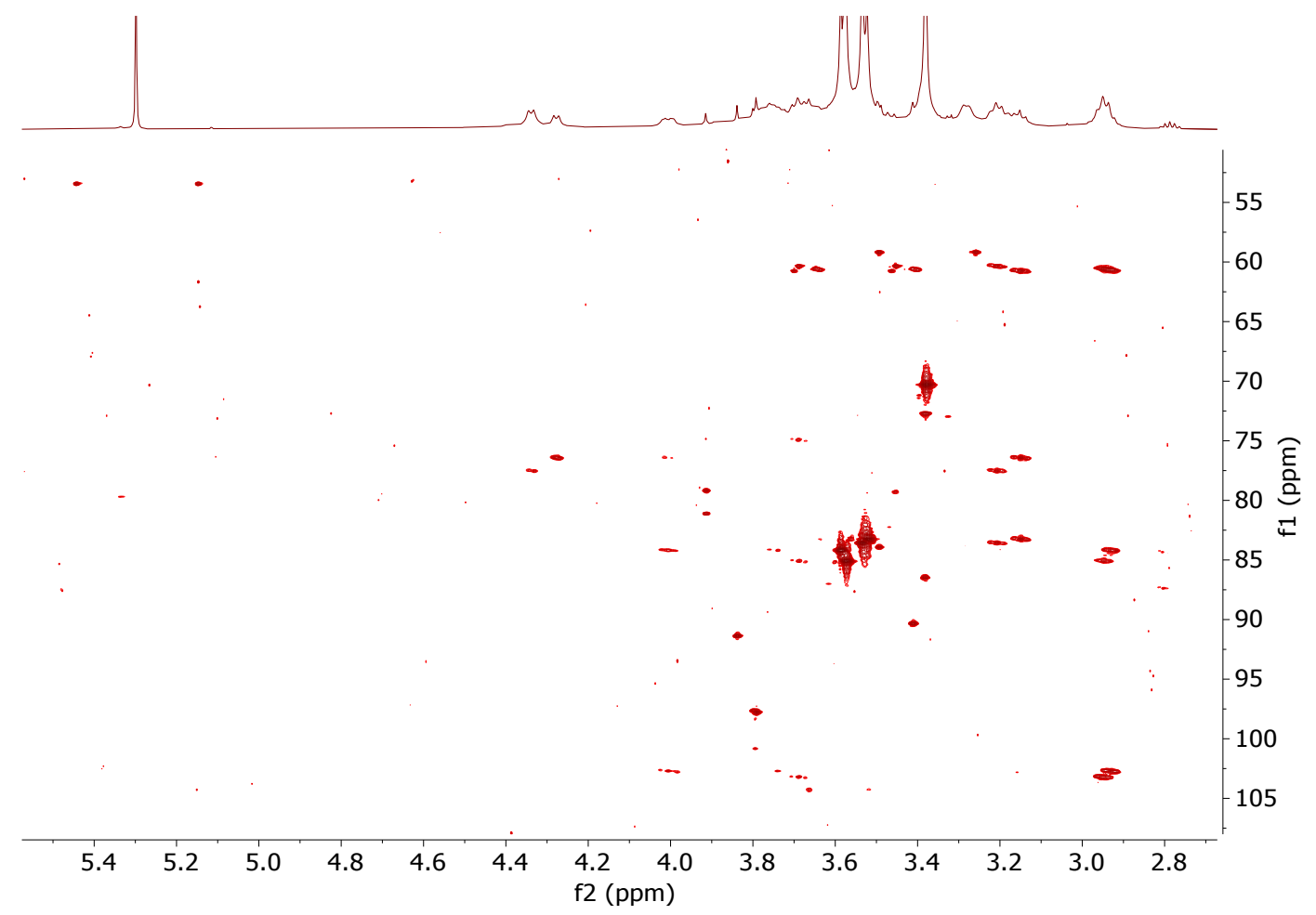

Figure S6: HMBC NMR spectrum of permethylated switchgrass sample $(\sim 5.8 \mathrm{mg})$ acquired in $600 \mu \mathrm{L}$ $\mathrm{CDCl}_{3}$ with $1536 * 512(\mathrm{~F} 2 * \mathrm{~F} 1)$ data points on a Bruker $600 \mathrm{MHz}$ spectrometer quipped with a $5 \mathrm{~mm}$ TXO cryoprobe. Spectrum acquired with 20 scans per increment.

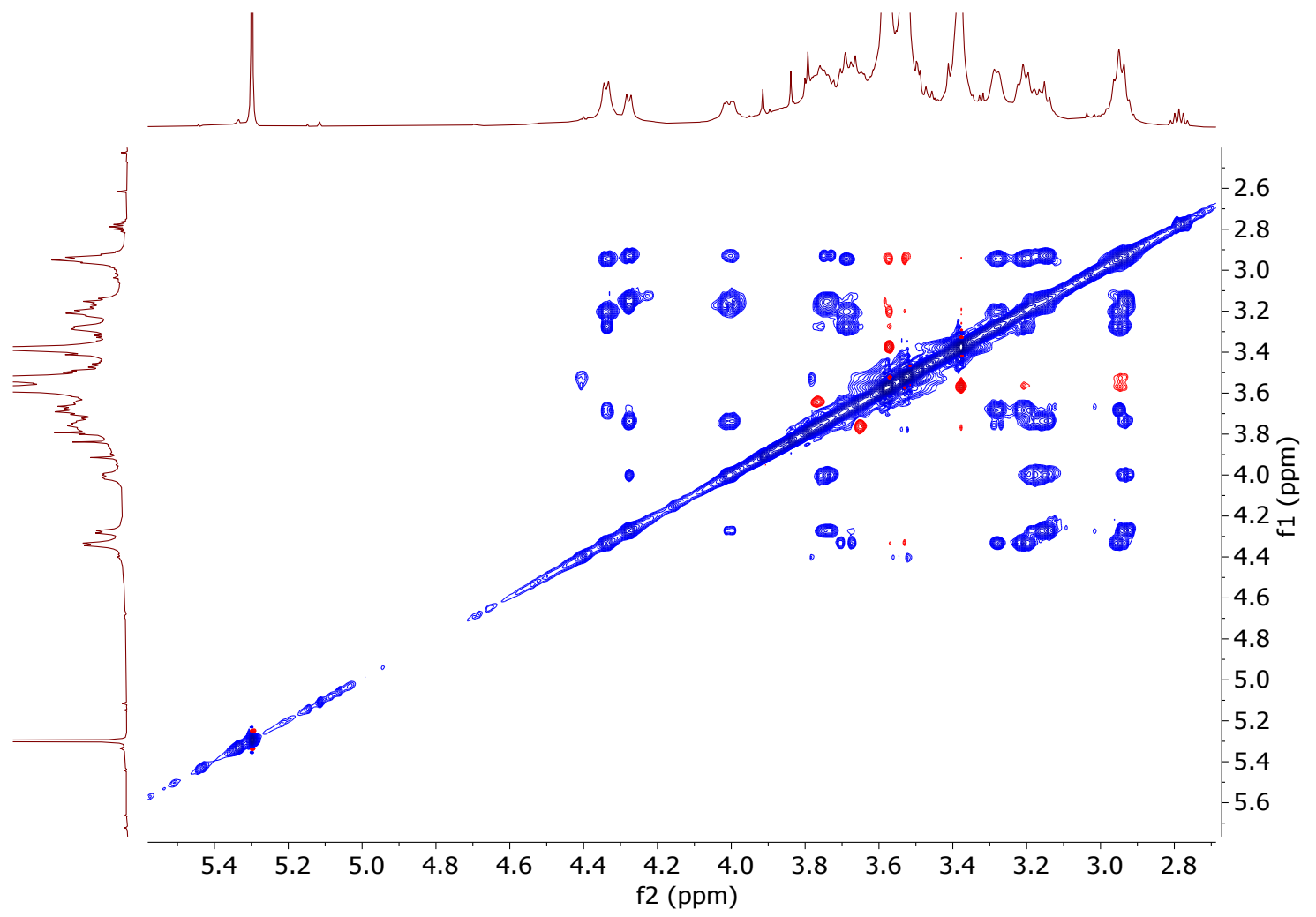

Figure S7: TOCSY NMR spectrum of permethylated switchgrass sample $(\sim 5.8 \mathrm{mg})$ acquired in 600 $\mu \mathrm{L} \mathrm{CDCl}{ }_{3}$ with $1024 * 420(\mathrm{~F} 2 * \mathrm{~F} 1)$ data points on a Bruker $600 \mathrm{MHz}$ spectrometer quipped with a 5 $\mathrm{mm}$ TXO cryoprobe. $80 \mathrm{~ms}$ spin-lock time. Spectrum acquired with 8 scans per increment. 


\section{Peracetylated Switchgrass NMR Data}

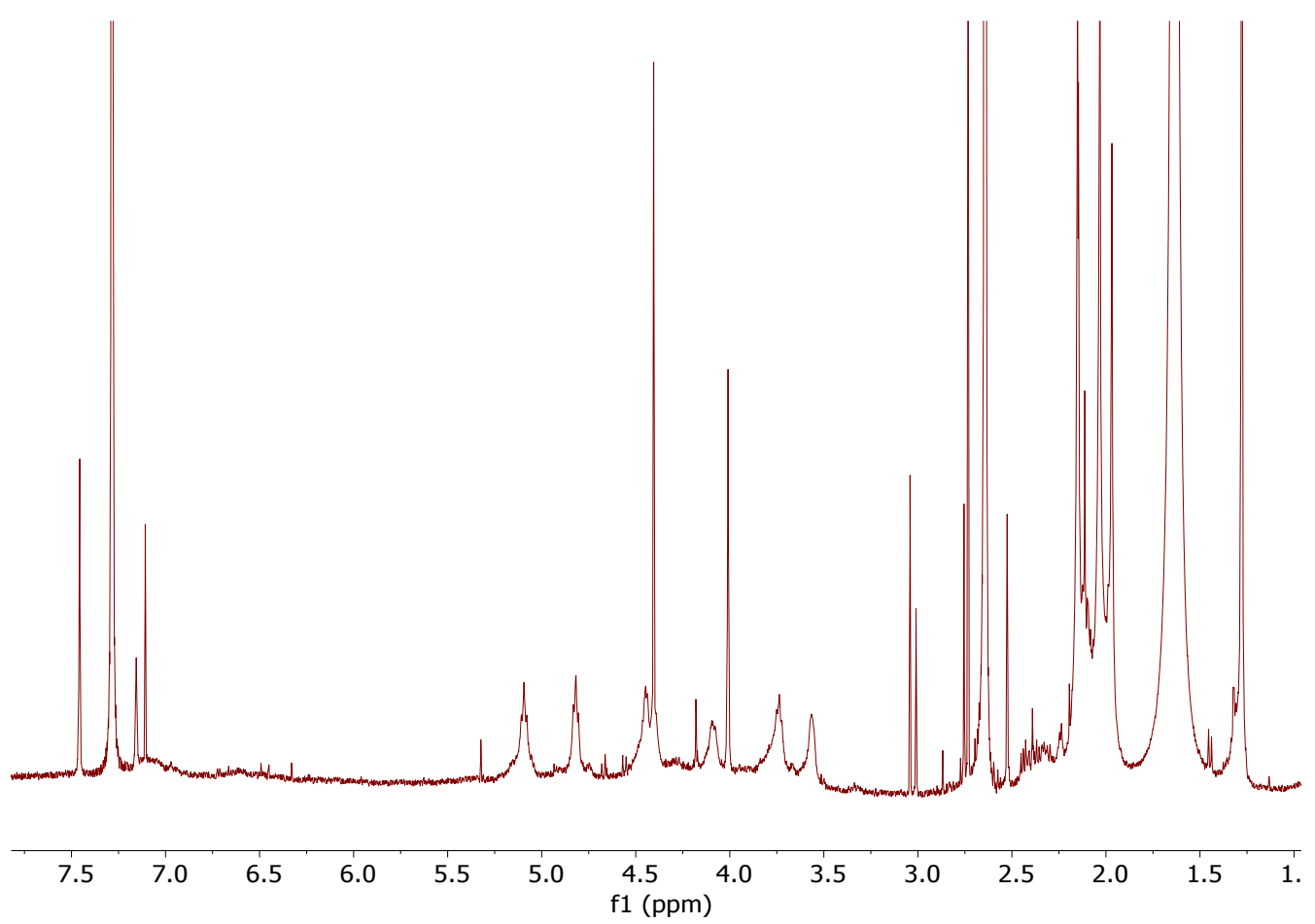

Figure S8: ${ }^{1} \mathrm{H}$ NMR spectrum of peracetylated switchgrass sample $(\sim 5.8 \mathrm{mg})$ acquired in $600 \mu \mathrm{L}$ $\mathrm{CDCl}_{3}$ on a Bruker $600 \mathrm{MHz}$ spectrometer quipped with a $5 \mathrm{~mm}$ TCI cryoprobe. Spectrum acquired with 120 scans.

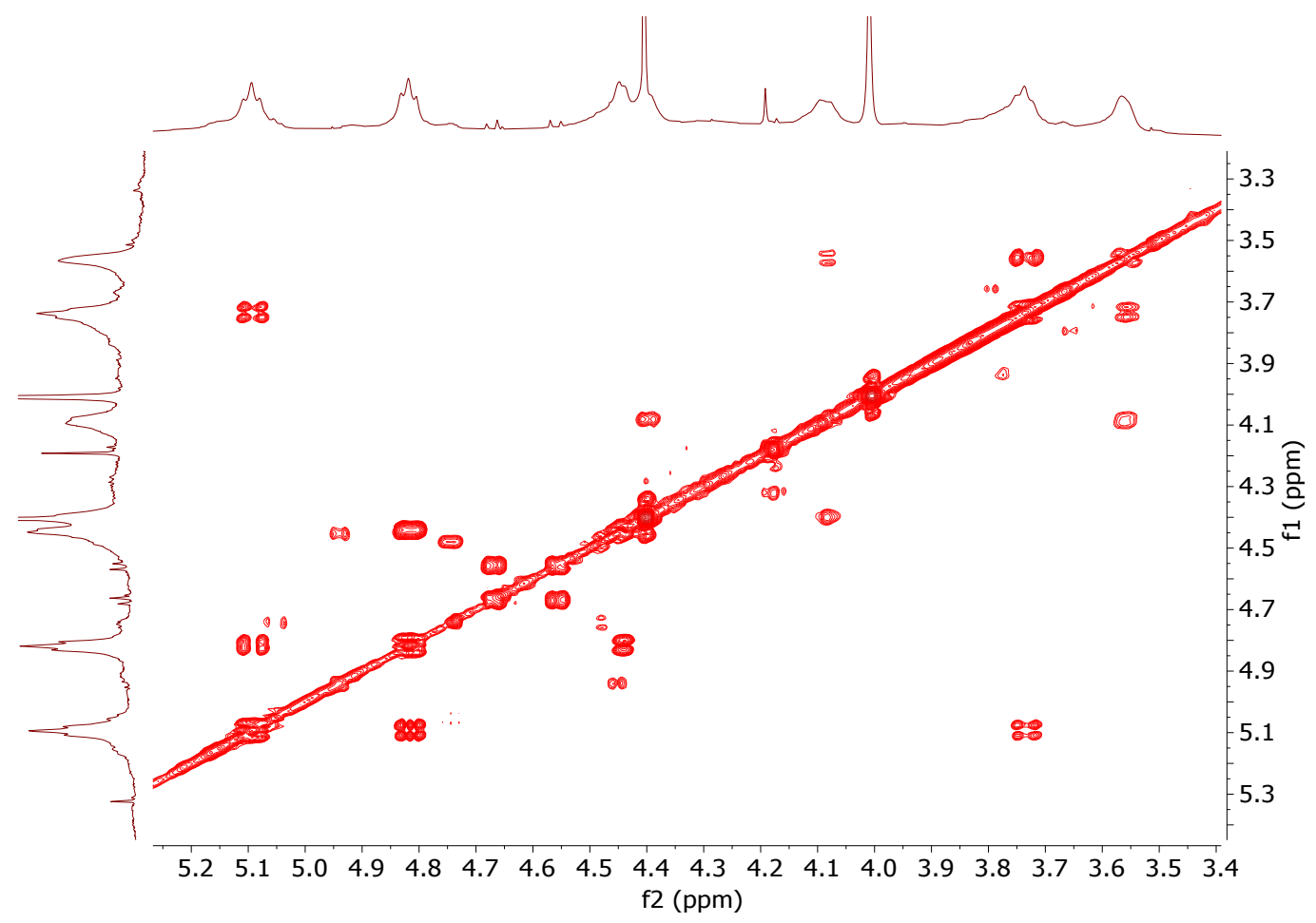

Figure S9: COSY NMR spectrum of peracetylated switchgrass sample $(\sim 5.8 \mathrm{mg})$ acquired in $600 \mu \mathrm{L}$ $\mathrm{CDCl}_{3}$ with $1024 * 512(\mathrm{~F} 2 * \mathrm{~F} 1)$ data points on a Bruker $600 \mathrm{MHz}$ spectrometer quipped with a $5 \mathrm{~mm}$ TCI cryoprobe. Spectrum acquired with 12 scans per increment. 


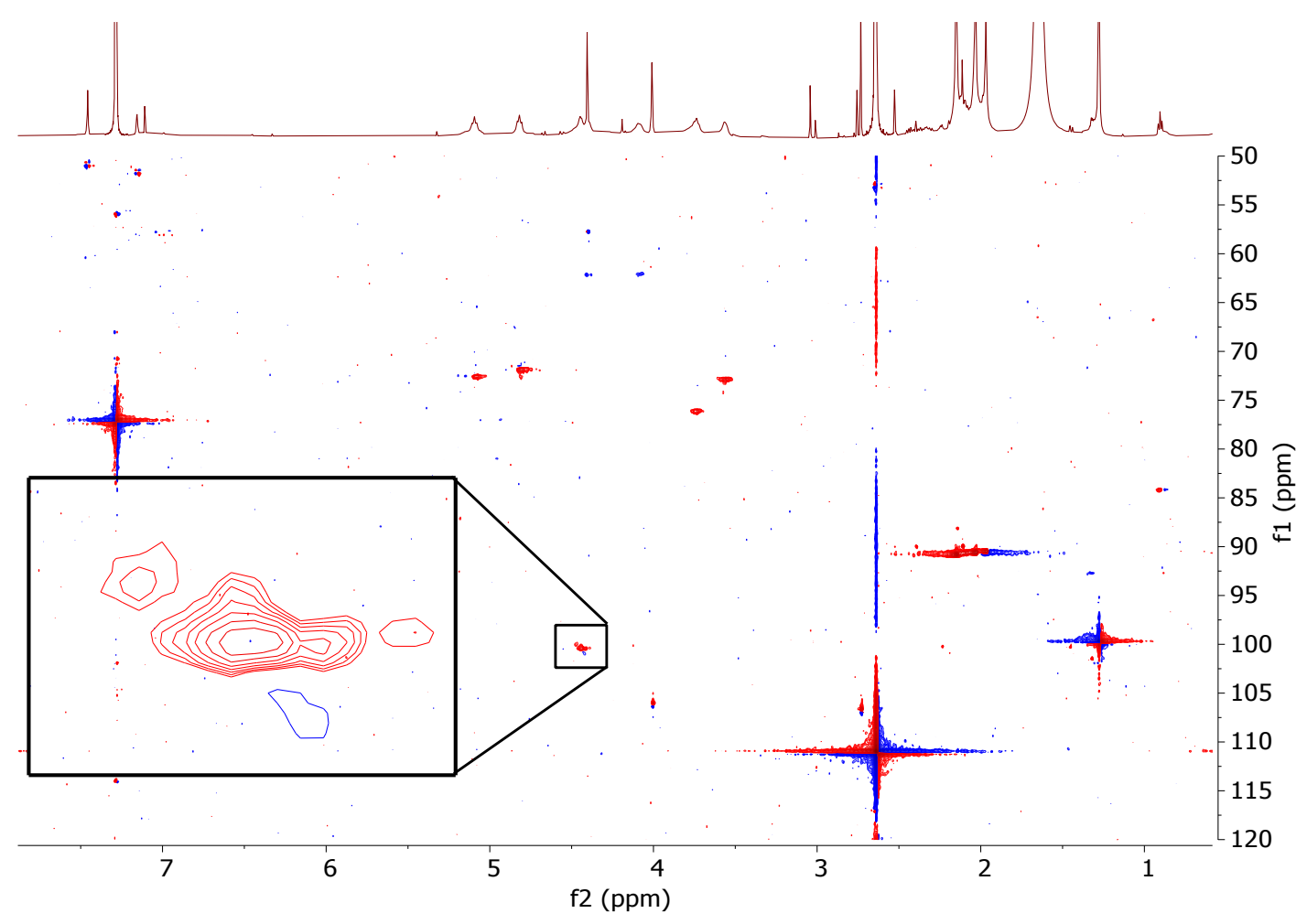

Figure S10: HSQC NMR spectrum of peracetylated switchgrass sample $(\sim 5.8 \mathrm{mg})$ acquired in $600 \mu \mathrm{L}$ $\mathrm{CDCl}_{3}$ with $768 * 512(\mathrm{~F} 2 * \mathrm{~F} 1)$ data points on a Bruker $600 \mathrm{MHz}$ spectrometer quipped with a $5 \mathrm{~mm}$ TCI cryoprobe. Spectrum acquired with 24 scans per increment.

\section{Permethylated Poplar NMR Data}

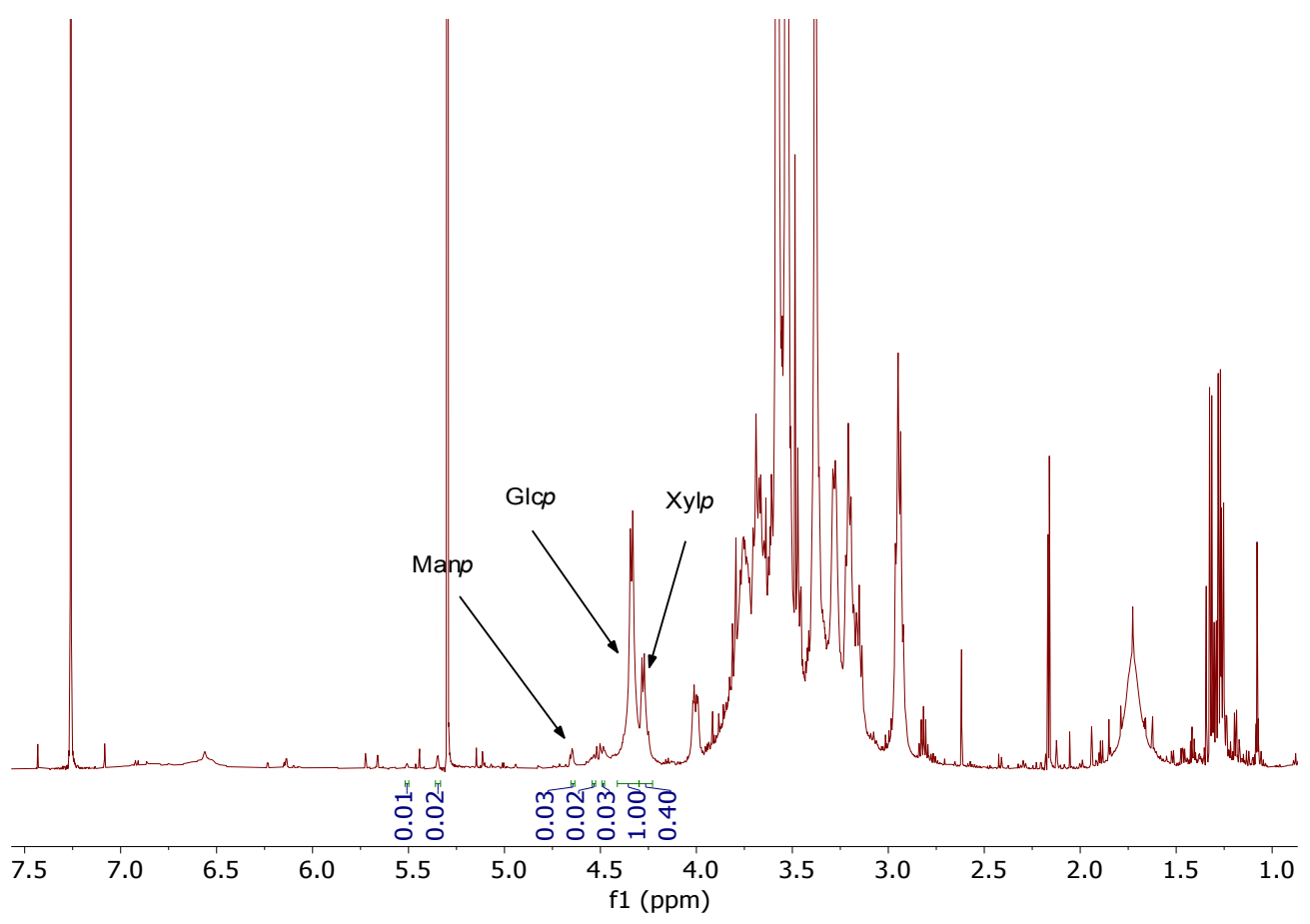

Figure S11: ${ }^{1} \mathrm{H}$ NMR spectrum of permethylated poplar sample $(\sim 5.8 \mathrm{mg})$ acquired in $600 \mu \mathrm{L} \mathrm{CDCl}{ }_{3}$ on a Bruker $600 \mathrm{MHz}$ spectrometer quipped with a $5 \mathrm{~mm}$ TCI cryoprobe. Recycle delay $30 \mathrm{~ms}$. Spectrum acquired with 2 scans. 


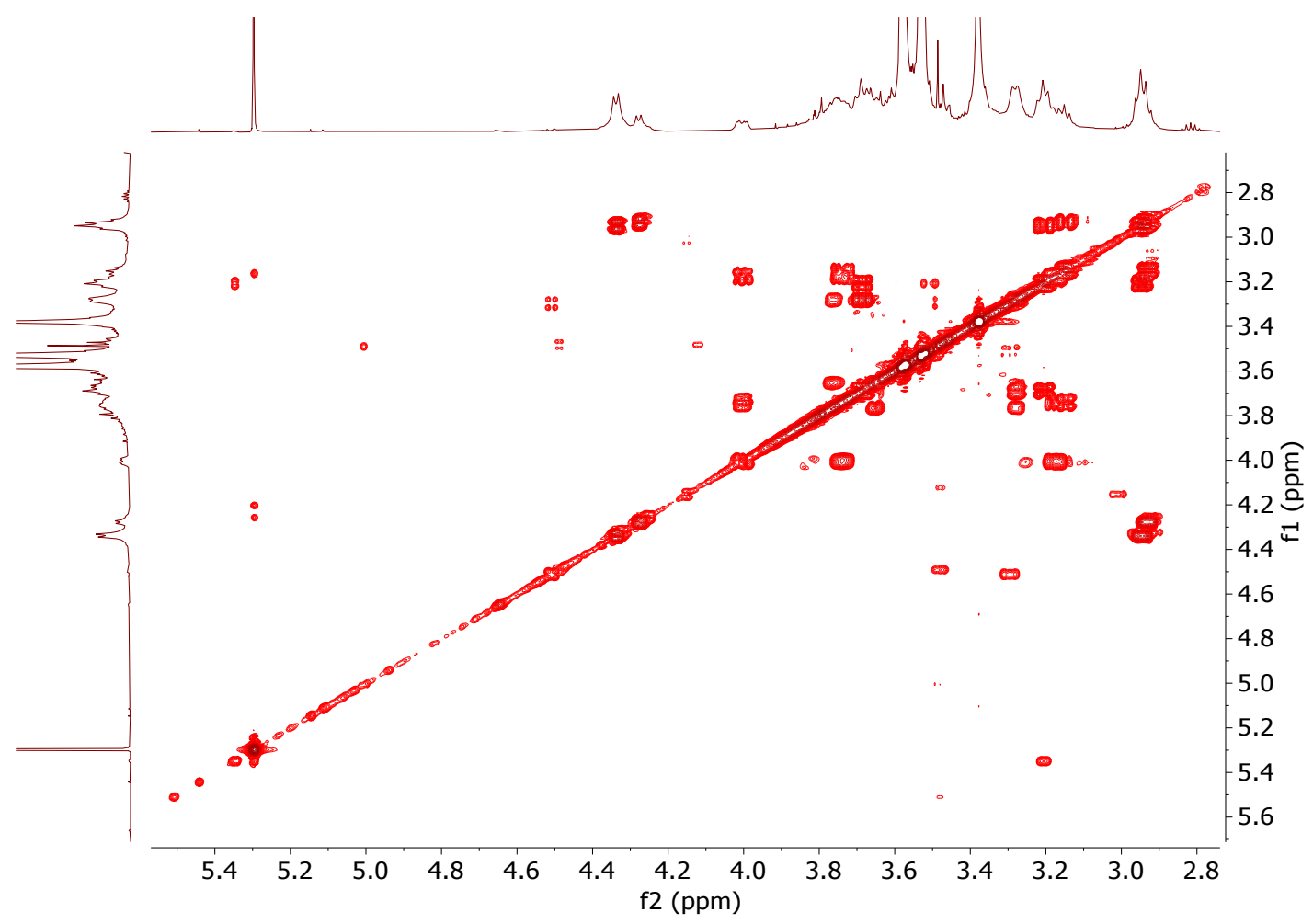

Figure S12: COSY NMR spectrum of permethylated poplar sample $(\sim 5.8 \mathrm{mg})$ acquired in $600 \mu \mathrm{L}$ $\mathrm{CDCl}_{3}$ with $1024 * 512(\mathrm{~F} 2 * \mathrm{~F} 1)$ data points on a Bruker $600 \mathrm{MHz}$ spectrometer quipped with a $5 \mathrm{~mm}$ TCI cryoprobe. Spectrum was acquired with 6 scans per increment.

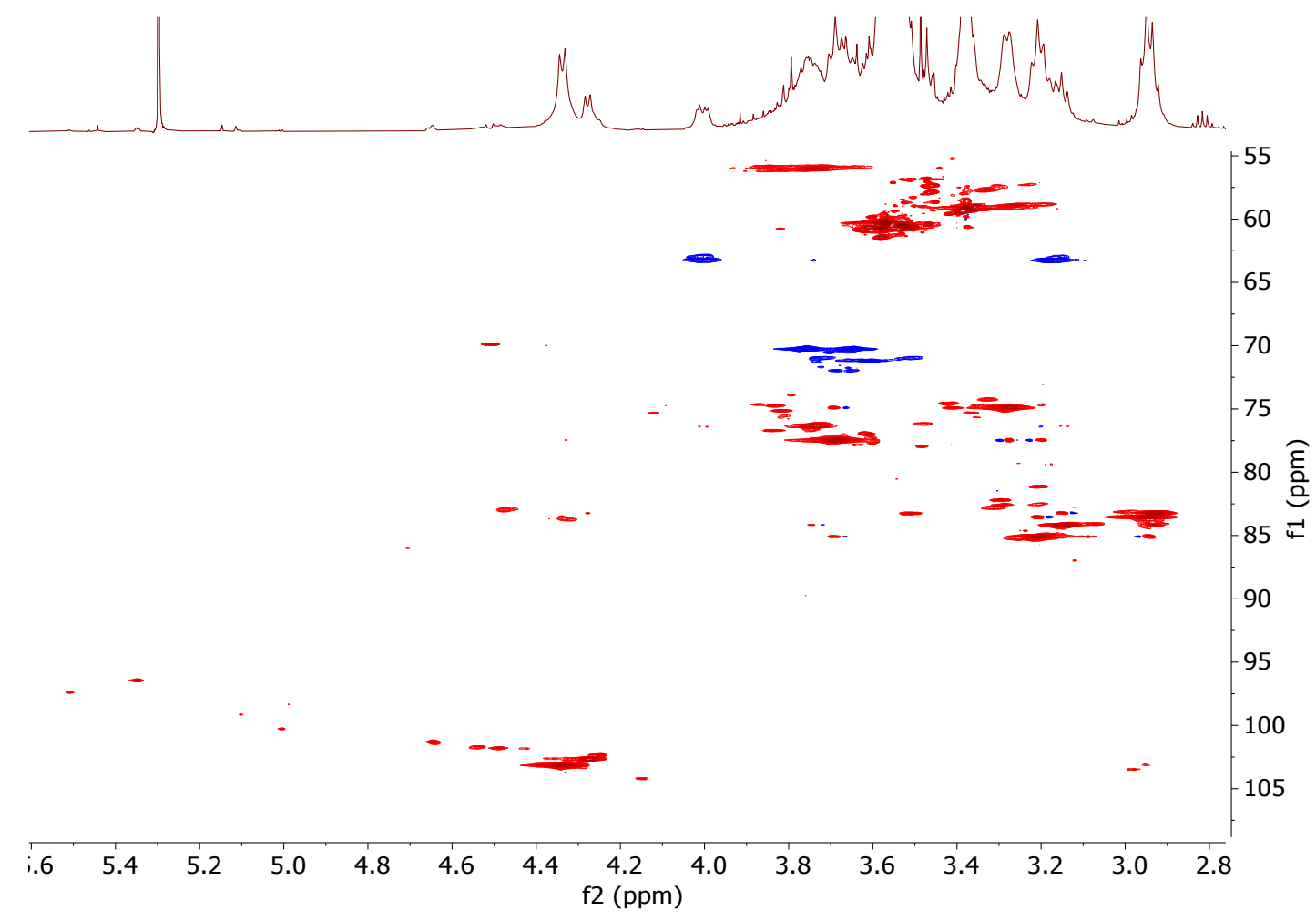

Figure S13: HSQC NMR spectrum of permethylated poplar sample $(\sim 5.8 \mathrm{mg})$ acquired in $600 \mu \mathrm{L}$ $\mathrm{CDCl}_{3}$ with $768 * 768(\mathrm{~F} 2 * \mathrm{~F} 1)$ data points on a Bruker $600 \mathrm{MHz}$ spectrometer quipped with a $5 \mathrm{~mm}$ TCI cryoprobe. Spectrum acquired with 8 scans per increment. 


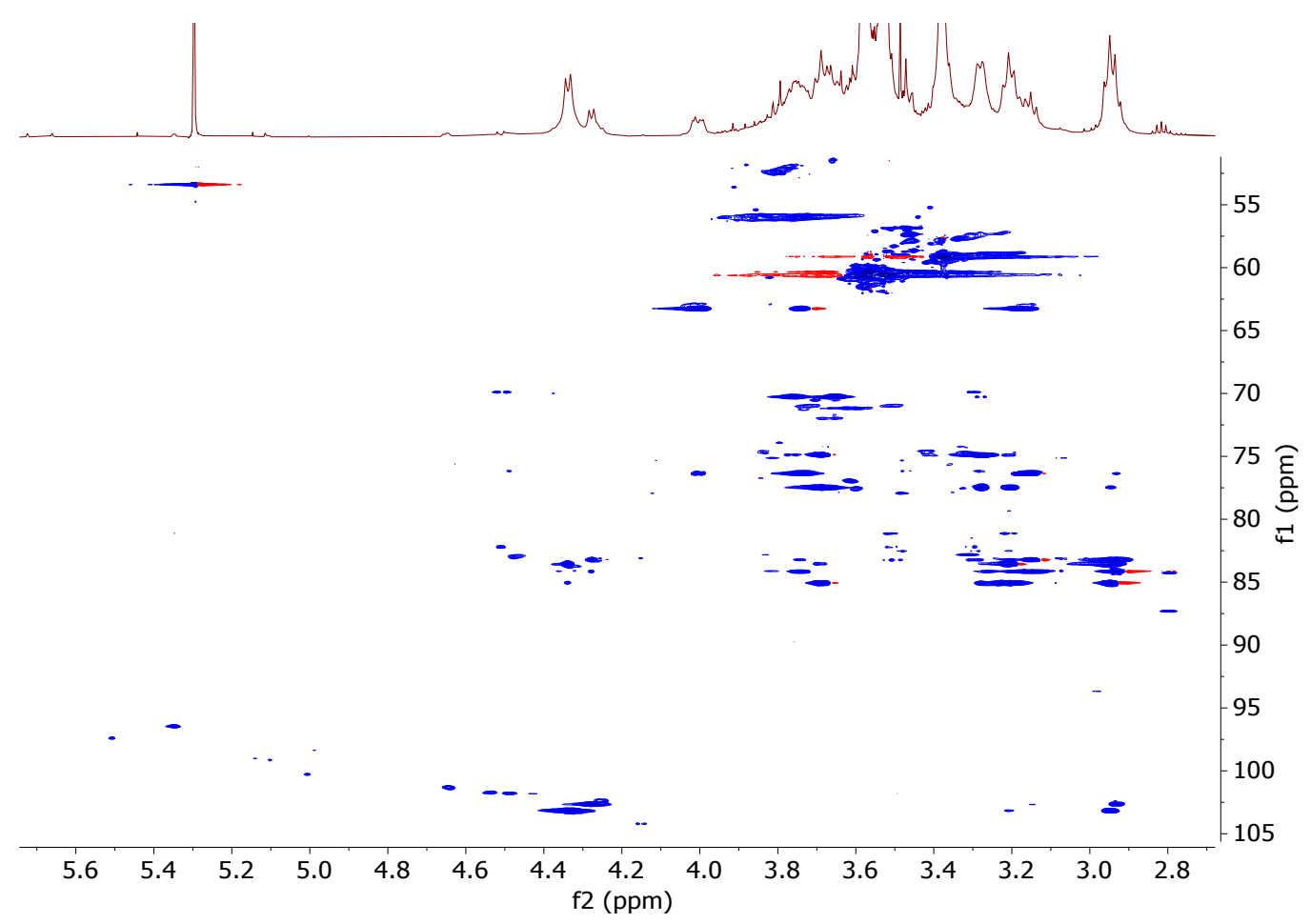

Figure S14: HSQC-TOCSY NMR spectrum of permethylated poplar sample ( $\sim 5.8 \mathrm{mg})$ acquired in $600 \mu \mathrm{L} \mathrm{CDCl}_{3}$ with $768 * 768(\mathrm{~F} 2 * \mathrm{~F} 1)$ data points on a Bruker $600 \mathrm{MHz}$ spectrometer quipped with a $5 \mathrm{~mm}$ TCI cryoprobe. Spin-lock time of $18 \mathrm{~ms}$. Spectrum acquired with 16 scans per increment.

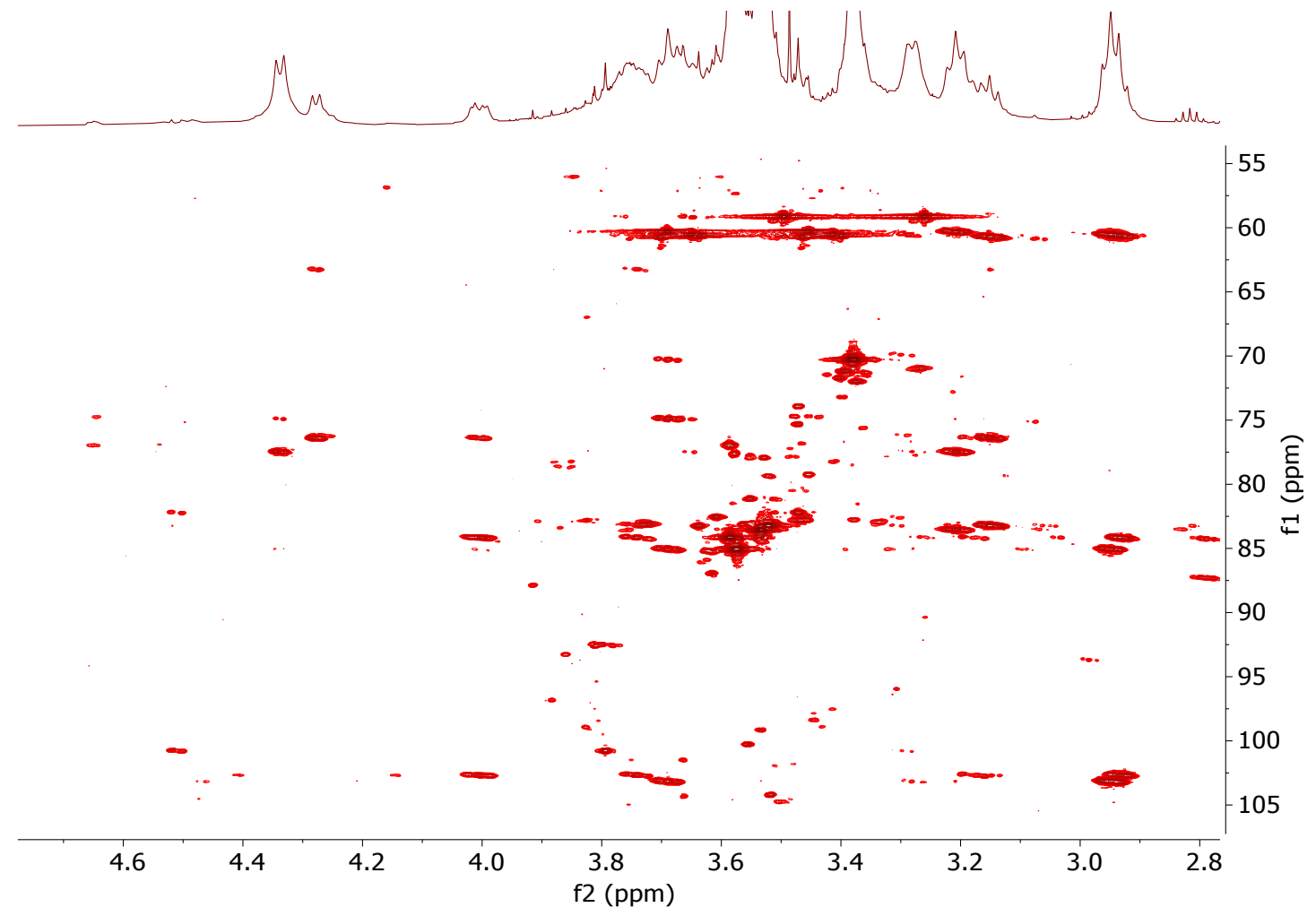

Figure S15: HMBC NMR spectrum of permethylated poplar sample $(\sim 5.8 \mathrm{mg})$ acquired in $600 \mu \mathrm{L}$ $\mathrm{CDCl}_{3}$ with $1536 * 256(\mathrm{~F} 2 * \mathrm{~F} 1)$ data points on a Bruker $600 \mathrm{MHz}$ spectrometer quipped with a $5 \mathrm{~mm}$ TCI cryoprobe. Spectrum acquired with 32 scans per increment. 


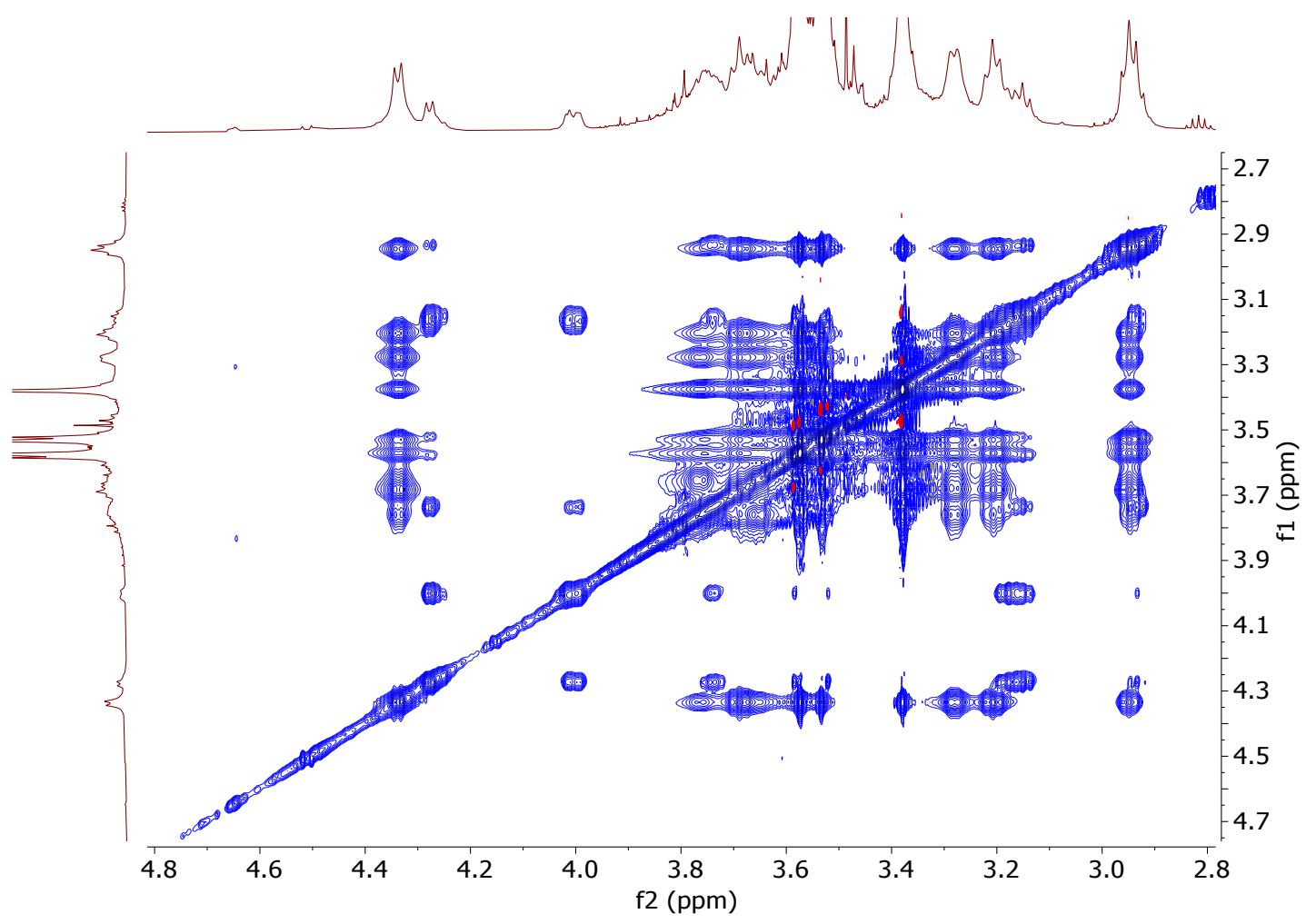

Figure S16: NOESY NMR full spectrum of permethylated poplar sample $(\sim 5.8 \mathrm{mg})$ acquired in 600 $\mu \mathrm{L} \mathrm{CDCl}{ }_{3}$ with $1024 * 512(\mathrm{~F} 2 * \mathrm{~F} 1)$ data points on a Bruker $600 \mathrm{MHz}$ spectrometer quipped with a 5 $\mathrm{mm}$ TCI cryoprobe. Spectrum acquired with 8 scans per increment.

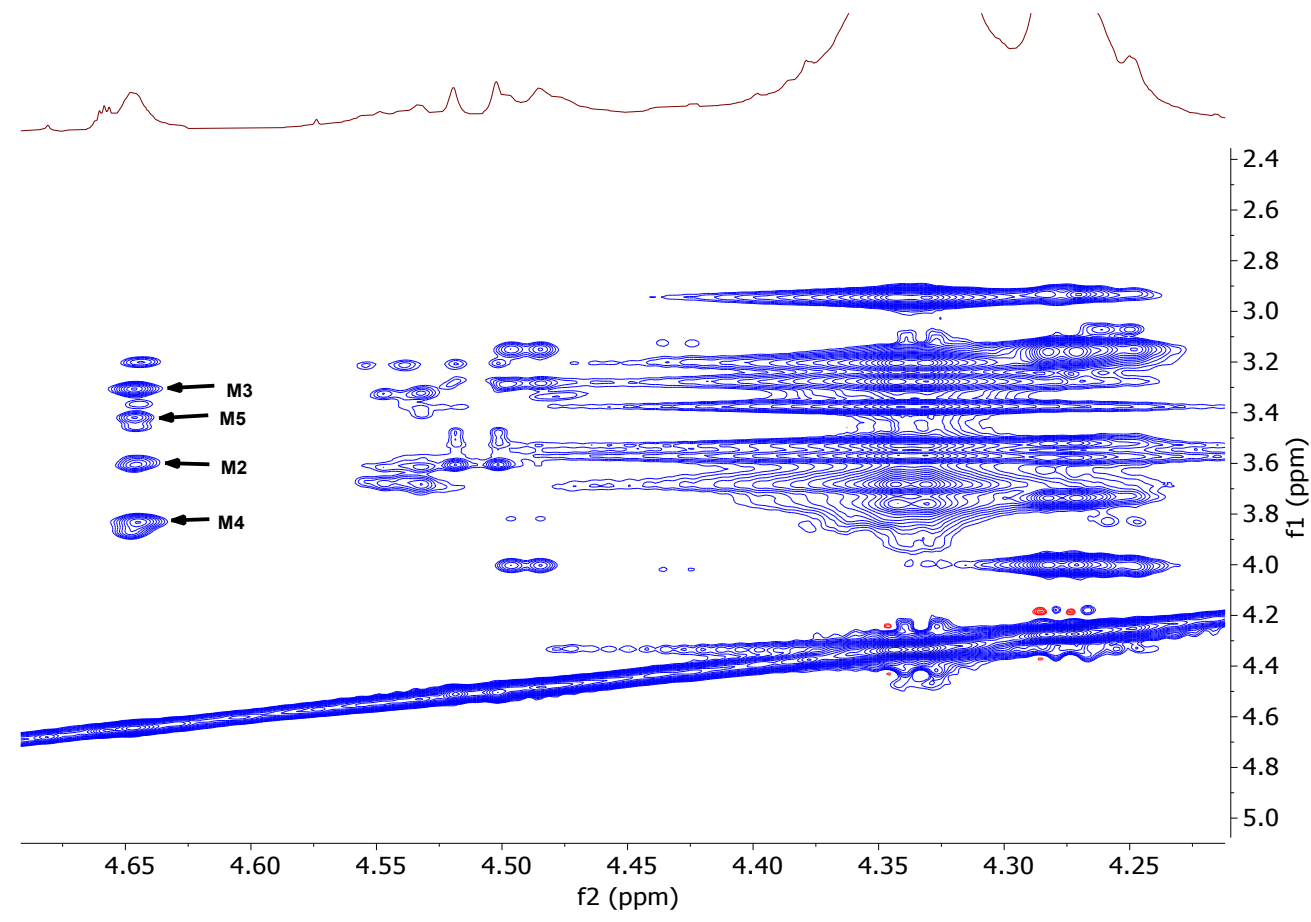

Figure S17: NOESY NMR expanded/zoomed-in section of permethylated poplar sample $(\sim 5.8 \mathrm{mg})$ acquired in $600 \mu \mathrm{L} \mathrm{CDCl}_{3}$ with $1024 * 512(\mathrm{~F} 2 * \mathrm{~F} 1)$ data points on a Bruker $600 \mathrm{MHz}$ spectrometer quipped with a $5 \mathrm{~mm}$ TCI cryoprobe. The weak mannose anomeric proton NOE correlation is shown (marked with 'M'). Spectrum acquired with 8 scans per increment. 


\section{Chemical Shift Table of Permethylated Polysaccharides}

Table S 1: ${ }^{1} \mathrm{H}$ and ${ }^{13} \mathrm{C}$ chemical shifts of permethylated homo-polysaccharides. Chemical shifts are referenced to residual chloroform- $d$ solvent peaks at 7.26 ppm $\left({ }^{1} \mathrm{H}\right)$ and $77.36 \mathrm{ppm}\left({ }^{13} \mathrm{C}\right)$.

\begin{tabular}{|c|c|c|c|c|c|c|c|c|c|}
\hline & 1 & 2 & 3 & 4 & 5 & 6 & $\mathrm{OMe}\left({ }^{*}\right)$ & $\mathrm{OMe}\left({ }^{*}\right)$ & OMe $(*)$ \\
\hline \multicolumn{10}{|c|}{ 1,5-L-Arabinan } \\
\hline \multicolumn{10}{|l|}{$\rightarrow 5)-\alpha$-L-Araf $(1 \rightarrow 5$} \\
\hline${ }^{1} \mathbf{H}$ & 5.06 & 3.76 & 3.68 & 4.06 & $3.69 / 3.82$ & & $3.38(2)$ & $3.39(3)$ & \\
\hline$\Delta \delta_{\mathrm{H}}{ }^{* *}$ & -0.04 & -0.39 & -0.37 & -0.16 & $-0.13 /-0.08$ & & & & \\
\hline${ }^{13} \mathrm{C}$ & 106.0 & 89.9 & 85.2 & 80.9 & 66.1 & & 57.6 & 58.2 & \\
\hline$\Delta \delta_{c}^{* *}$ & -1.8 & 8.4 & 8.4 & -1.6 & -0.7 & & & & \\
\hline & & & & & & & & & \\
\hline \multicolumn{10}{|c|}{ 1,4-D-Xylan } \\
\hline \multicolumn{10}{|l|}{$\rightarrow 4)-\beta$-D-Xylp $(1 \rightarrow 4$} \\
\hline${ }^{1} \mathbf{H}$ & 4.28 & 2.94 & 3.15 & 3.74 & $3.18 / 4.01$ & & $3.52(2)$ & 3.59 (3) & \\
\hline$\Delta \delta_{H}^{* * *}$ & -0.26 & -0.43 & -0.51 & -0.16 & $-0.28 /-0.13$ & & & & \\
\hline${ }^{13} \mathrm{C}$ & 102.8 & 83.3 & 84.3 & 76.5 & 63.3 & & 60.7 & 60.8 & \\
\hline$\Delta \delta_{C}^{* * *}$ & 0.2 & 9.9 & 9.5 & -0.9 & -0.8 & & & & \\
\hline \multicolumn{10}{|c|}{ 1,4-D-Mannan } \\
\hline \multicolumn{10}{|l|}{$\rightarrow 4)-\beta$-D-Man $p(1 \rightarrow 4$} \\
\hline${ }^{1} \mathbf{H}$ & 4.65 & 3.62 & 3.31 & 3.84 & 3.43 & $3.64 / 3.68$ & $3.59(2)$ & $3.46(3)$ & $3.37(6)$ \\
\hline$\Delta \delta_{H}^{* * *}$ & -0.08 & -0.45 & -0.49 & 0.01 & -0.07 & $-0.08 /-0.20$ & & & \\
\hline${ }^{13} \mathrm{C}$ & 101.3 & 77.0 & 82.8 & 74.7 & 74.6 & 72.0 & 61.5 & 57.3 & 59.3 \\
\hline$\Delta \delta_{C}^{* * *}$ & 0.5 & 5.8 & 10.6 & -1.9 & -1.4 & 10.0 & & & \\
\hline & & & & & & & & & \\
\hline \multicolumn{10}{|c|}{ Cellulose } \\
\hline$\rightarrow 4)-\beta$-D-Glc $p(1 \rightarrow 4$ & & & & & & & & & \\
\hline
\end{tabular}




\begin{tabular}{|c|c|c|c|c|c|c|c|c|c|}
\hline${ }^{1} \mathbf{H}$ & 4.34 & 2.95 & 3.21 & 3.69 & 3.28 & $3.65 / 3.76$ & $3.53(2)$ & $3.57(3)$ & $3.38(6)$ \\
\hline$\Delta \delta_{\mathrm{H}} * * *$ & -0.2 & -0.44 & -0.45 & 0.05 & -0.37 & $-0.17 /-0.23$ & & & \\
\hline${ }^{13} \mathrm{C}$ & 103.2 & 83.5 & 85.0 & 77.4 & 74.8 & 70.2 & 60.5 & 60.3 & 59.1 \\
\hline$\Delta \delta_{C}^{* * *}$ & 0.0 & 9.6 & 9.9 & -2.3 & -0.9 & 9.1 & & & \\
\hline \multicolumn{10}{|c|}{ Starch } \\
\hline \multicolumn{10}{|l|}{$\rightarrow 4)-\alpha-\mathrm{D}-\mathrm{Glc} p(1 \rightarrow 4$} \\
\hline${ }^{1} \mathbf{H}$ & 5.57 & 3.24 & 3.58 & 3.84 & 3.67 & $3.49 / 3.77$ & $3.51(2)$ & $3.56(3)$ & $3.38(6)$ \\
\hline$\Delta \delta_{H} * * *$ & 0.21 & -0.4 & -0.37 & 0.2 & -0.17 & $-0.32 /-0.12$ & & & \\
\hline${ }^{13} \mathrm{C}$ & 97.1 & 82.4 & 83.2 & 73.7 & 70.5 & 71.0 & 59.2 & 60.4 & 59.5 \\
\hline$\Delta \delta_{\mathrm{C}}^{* * *}$ & -3.3 & 9.9 & 9.1 & -4.5 & -1.7 & 9.5 & & & \\
\hline \multicolumn{10}{|c|}{ Dextran } \\
\hline \multicolumn{10}{|l|}{$\rightarrow 6)-\alpha-\mathrm{D}-\mathrm{Glc} p(1 \rightarrow 6$} \\
\hline $\begin{array}{ll} & \\
\end{array}$ & 4.96 & 3.14 & 3.49 & 3.23 & 3.68 & $3.66 / 3.81$ & $3.45(2)$ & $3.6(3)$ & $3.53(4)$ \\
\hline$\Delta \delta_{H} * * *$ & -0.01 & -0.45 & -0.25 & -0.31 & -0.22 & $-0.11 /-0.17$ & & & \\
\hline${ }^{13} \mathrm{C}$ & 96.6 & 82.0 & 83.5 & 79.8 & 70.4 & 66.2 & 58.4 & 60.8 & 60.6 \\
\hline$\Delta \delta_{\mathrm{C}}^{* * *}$ & -2.3 & 9.6 & 9.2 & 9.1 & -0.9 & -0.7 & & & \\
\hline \multicolumn{10}{|c|}{ 1,4-D-Galactan } \\
\hline \multicolumn{10}{|l|}{$\rightarrow 4)-\beta-\mathrm{D}-\mathrm{Gal} p(1 \rightarrow 4$} \\
\hline${ }^{1} \mathbf{H}$ & 4.70 & 3.25 & 3.14 & 4.25 & 3.43 & $3.44 / 3.54$ & $3.61(2)$ & $3.46(3)$ & $3.28(6)$ \\
\hline$\Delta \delta_{H}^{* * *}$ & -0.04 & -0.84 & -0.62 & 0.45 & -0.11 & $-0.38 /-0.45$ & & & \\
\hline${ }^{13} \mathrm{C}$ & 102.3 & 81.9 & 83.6 & 69.0 & 72.2 & 72.3 & 60.6 & 58.8 & 58.1 \\
\hline$\Delta \delta_{\mathrm{C}}^{* * *}$ & 1.6 & 10.7 & 11.3 & -7.8 & -3.9 & 11.0 & & & \\
\hline $\begin{array}{l}* \text { Methoxy position } \\
* * \text { Compared to literature } \\
* * * \text { Compared to CASPER }\end{array}$ & $\begin{array}{l}\mathrm{f} \text { the no } \\
\text { redictec }\end{array}$ & methy & d equ & lents 6 & 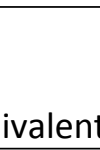 & & & & \\
\hline
\end{tabular}


Table S 2: ${ }^{1} \mathrm{H}$ and ${ }^{13} \mathrm{C}$ chemical shifts of permethylated polysaccharides containing multiple different of ring residues. Chemical shifts are referenced to residual chloroform- $d$ solvent peaks at $7.26 \mathrm{ppm}\left({ }^{1} \mathrm{H}\right)$ and $77.36 \mathrm{ppm}\left({ }^{13} \mathrm{C}\right)$.

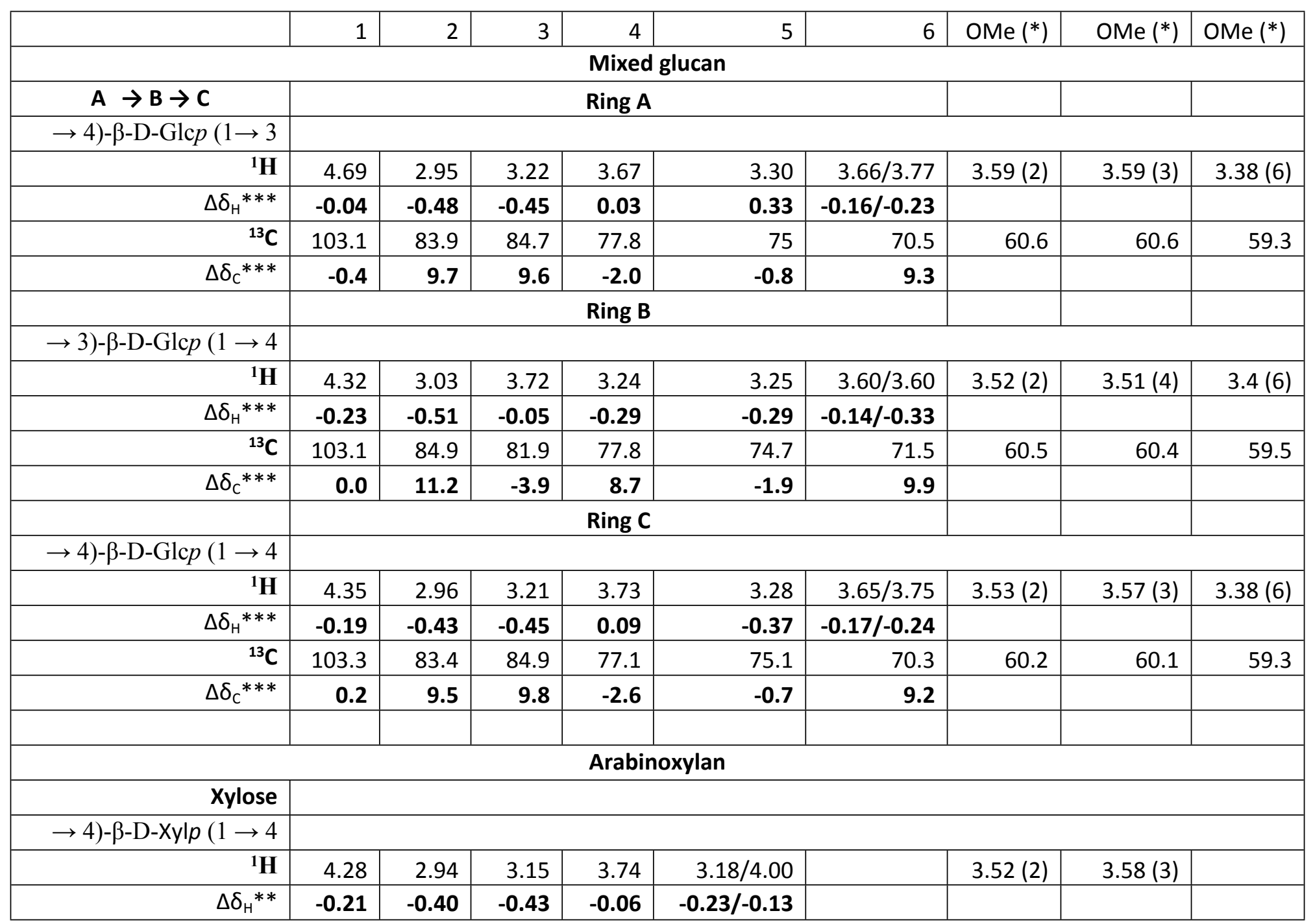




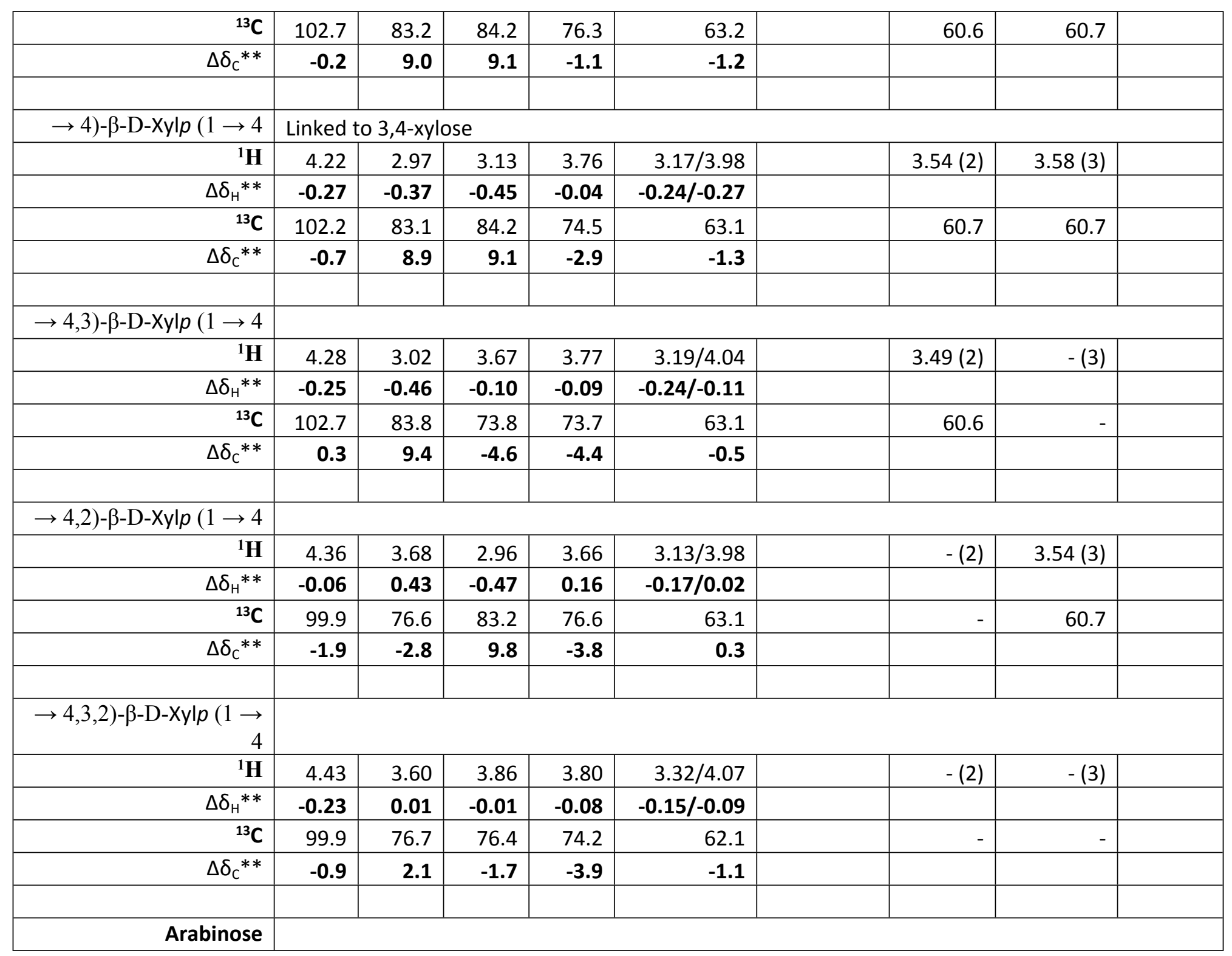




\begin{tabular}{|c|c|c|c|c|c|c|c|c|c|}
\hline \multicolumn{10}{|l|}{$\alpha$-L-Araf $\rightarrow(3-\beta-X y l p$} \\
\hline${ }^{1} \mathrm{H}$ & 5.33 & 3.78 & 3.53 & 4.41 & $3.51 / 3.55$ & & $3.41(2)$ & $3.38(3)$ & $3.38(5)$ \\
\hline$\Delta \delta_{H}^{* *}$ & -0.08 & -0.39 & -0.40 & 0.23 & $-0.22 /-0.25$ & & & & \\
\hline${ }^{13} \mathrm{C}$ & 105.5 & 90.3 & 86.4 & 79.7 & 72.7 & & 57.4 & 59.3 & 59.3 \\
\hline$\Delta \delta_{\mathrm{C}}^{* *}$ & -3.3 & 8.0 & 7.8 & -6.1 & 10.1 & & & & \\
\hline$\alpha$-L-Araf $\rightarrow(3-\beta-X y l p$ & \multicolumn{9}{|c|}{ As part of 2,3,4-xylose } \\
\hline${ }^{1} \mathbf{H}$ & 5.25 & 3.74 & 3.56 & 4.42 & $3.56 / 3.56$ & & $3.38(2)$ & $3.36(3)$ & $3.38(5)$ \\
\hline$\Delta \delta_{H}{ }^{* *}$ & -0.04 & -0.43 & -0.39 & 0.16 & $-0.17 /-0.23$ & & & & \\
\hline${ }^{13} \mathrm{C}$ & 105.9 & 89.9 & 85.8 & 80.5 & 72.7 & & 57.9 & 57.8 & 59.3 \\
\hline$\Delta \delta_{\mathrm{C}}^{* *}$ & -2.9 & 7.9 & 7.7 & -4.8 & 10.5 & & & & \\
\hline$\alpha$-L-Araf $\rightarrow(2-\beta-X y \mid p$ & \multicolumn{9}{|c|}{ As part of 2,4- or 2,3,4-xylose } \\
\hline${ }^{1} \mathrm{H}$ & 5.22 & 3.67 & 3.51 & 4.16 & $3.51 / 3.55$ & & $3.37(2)$ & $3.37(3)$ & $3.38(5)$ \\
\hline$\Delta \delta_{H} * *$ & -0.07 & -0.50 & -0.44 & -0.10 & $-0.22 /-0.24$ & & & & \\
\hline${ }^{13} \mathrm{C}$ & 106.2 & 89.5 & 85.9 & 81.3 & 72.7 & & 57.8 & $57.1 / 59.3$ & 59.3 \\
\hline$\Delta \delta_{C}^{* *}$ & -3.3 & 7.2 & 8.2 & -3.8 & 10.5 & & & & \\
\hline & & & & & & & & & \\
\hline \multicolumn{10}{|c|}{ Glucomannan } \\
\hline \multicolumn{10}{|c|}{$G_{A} \rightarrow M_{A} \rightarrow M_{C} \rightarrow G_{B} \rightarrow G_{C} \rightarrow M_{A} \rightarrow M_{B} \rightarrow M_{B} \rightarrow M_{C}$} \\
\hline Mannose & \multicolumn{6}{|c|}{ Ring A } & & & \\
\hline \multicolumn{10}{|l|}{$\rightarrow 4)-\beta-\mathrm{D}-\mathrm{Man} p(1 \rightarrow 4$} \\
\hline${ }^{1} \mathbf{H}$ & 4.65 & 3.61 & 3.2 & 3.82 & 3.36 & $3.7 / 3.73$ & $3.58(2)$ & $3.46(3)$ & $3.36(6)$ \\
\hline$\Delta \delta_{\mathrm{H}} * * *$ & -0.09 & -0.48 & -0.56 & 0.02 & -0.18 & $-0.12 /-0.26$ & & & \\
\hline${ }^{13} \mathrm{C}$ & 101.3 & 77.5 & 82.4 & 75.7 & 75.3 & 71.3 & 61.3 & 57.8 & 59.0 \\
\hline \multirow[t]{2}{*}{$\Delta \delta_{C}^{* * *}$} & 0.6 & 6.3 & 10.1 & -1.1 & -0.8 & 10.0 & & & \\
\hline & \multicolumn{6}{|c|}{ Ring B } & & & \\
\hline$\rightarrow 4)-\beta-\mathrm{D}-\mathrm{Man} p(1 \rightarrow 4$ & & & & & & & & & \\
\hline
\end{tabular}




\begin{tabular}{|c|c|c|c|c|c|c|c|c|c|}
\hline${ }^{1} \mathbf{H}$ & 4.65 & 3.61 & 3.3 & 3.83 & 3.42 & $3.66 / 3.69$ & $3.59(2)$ & $3.46(3)$ & $3.37(6)$ \\
\hline$\Delta \delta_{\mathrm{H}} * * *$ & -0.08 & -0.46 & -0.50 & 0.00 & -0.08 & $-0.06 /-0.19$ & & & \\
\hline${ }^{13} \mathrm{C}$ & 101.4 & 77.0 & 82.7 & 74.7 & 74.5 & 71.9 & 61.5 & 57.3 & 59.3 \\
\hline & \multicolumn{6}{|c|}{ Ring C } & & & \\
\hline$\Delta \delta_{\mathrm{H}} * * *$ & -0.19 & -0.45 & -0.48 & 0.04 & -0.09 & $-0.06 /-0.15$ & & & \\
\hline${ }^{13} \mathrm{C}$ & 101.7 & 76.9 & 82.8 & 74.6 & 74.9 & 71.7 & 61.4 & 57.4 & 59.4 \\
\hline$\Delta \delta_{C} * * *$ & 0.9 & 5.8 & 10.6 & -2.1 & -1.1 & 10.4 & & & \\
\hline$\Delta \delta_{H}^{* * *}$ & -0.17 & -0.42 & -0.43 & 0.02 & -0.28 & $-0.08 /-0.25$ & & & \\
\hline${ }^{13} \mathrm{C}$ & 103.1 & 84.1 & 85.2 & 77.4 & 74.2 & 71.2 & 60.4 & 60.3 & 59.4 \\
\hline$\Delta \delta_{C} * * *$ & -0.1 & 10.3 & 10.2 & -2.2 & -1.4 & 10.1 & & & \\
\hline & \multicolumn{6}{|c|}{ Ring B } & & & \\
\hline \multicolumn{10}{|l|}{$\rightarrow 4)-\beta-\mathrm{D}-\mathrm{Glc} p(1 \rightarrow 4$} \\
\hline${ }^{1} \mathbf{H}$ & 4.32 & 2.94 & 3.26 & 3.67 & 3.33 & $3.63 / 3.64$ & $3.53(2)$ & $3.62(3)$ & $3.39(6)$ \\
\hline$\Delta \delta_{\mathrm{H}} * * *$ & -0.21 & -0.43 & -0.43 & 0.00 & -0.28 & $-0.09 /-0.25$ & & & \\
\hline${ }^{13} \mathrm{C}$ & 103.1 & 84.1 & 85.2 & 77.6 & 74.3 & 71.2 & 60.4 & 60.6 & 59.4 \\
\hline $\begin{array}{ll}1 & \\
\end{array}$ & 4.35 & 2.95 & 3.2 & 3.71 & 3.27 & $3.66 / 3.74$ & $3.53(2)$ & $3.62(3)$ & $3.37(6)$ \\
\hline
\end{tabular}




\begin{tabular}{|l|r|r|r|r|r|r|r|r|r|}
\hline$\Delta \delta_{\mathrm{H}}{ }^{* * *}$ & $-\mathbf{0 . 1 9}$ & $-\mathbf{0 . 4 4}$ & $-\mathbf{0 . 4 6}$ & $\mathbf{0 . 0 7}$ & $\mathbf{- 0 . 3 8}$ & $\mathbf{- 0 . 1 6 / - 0 . 2 5}$ & & & \\
\hline${ }^{13} \mathrm{C}$ & 103.1 & 83.5 & 85.2 & 77.4 & 74.8 & 70.1 & 60.4 & 60.6 & 59.1 \\
\hline$\Delta \delta_{\mathrm{C}}{ }^{* * *}$ & $-\mathbf{0 . 1}$ & $\mathbf{9 . 6}$ & $\mathbf{1 0 . 1}$ & $-\mathbf{- 2 . 3}$ & $-\mathbf{- 1 . 0}$ & $\mathbf{9 . 0}$ & & & \\
\hline * Methoxy position \\
** Compared to literature of the non-methylated equivalents ${ }^{8-10}$ \\
$* * *$ Compared to CASPER ${ }^{7}$ predicted values of the non-methylated equivalents \\
\hline
\end{tabular}




\section{Arabinan NMR Data}
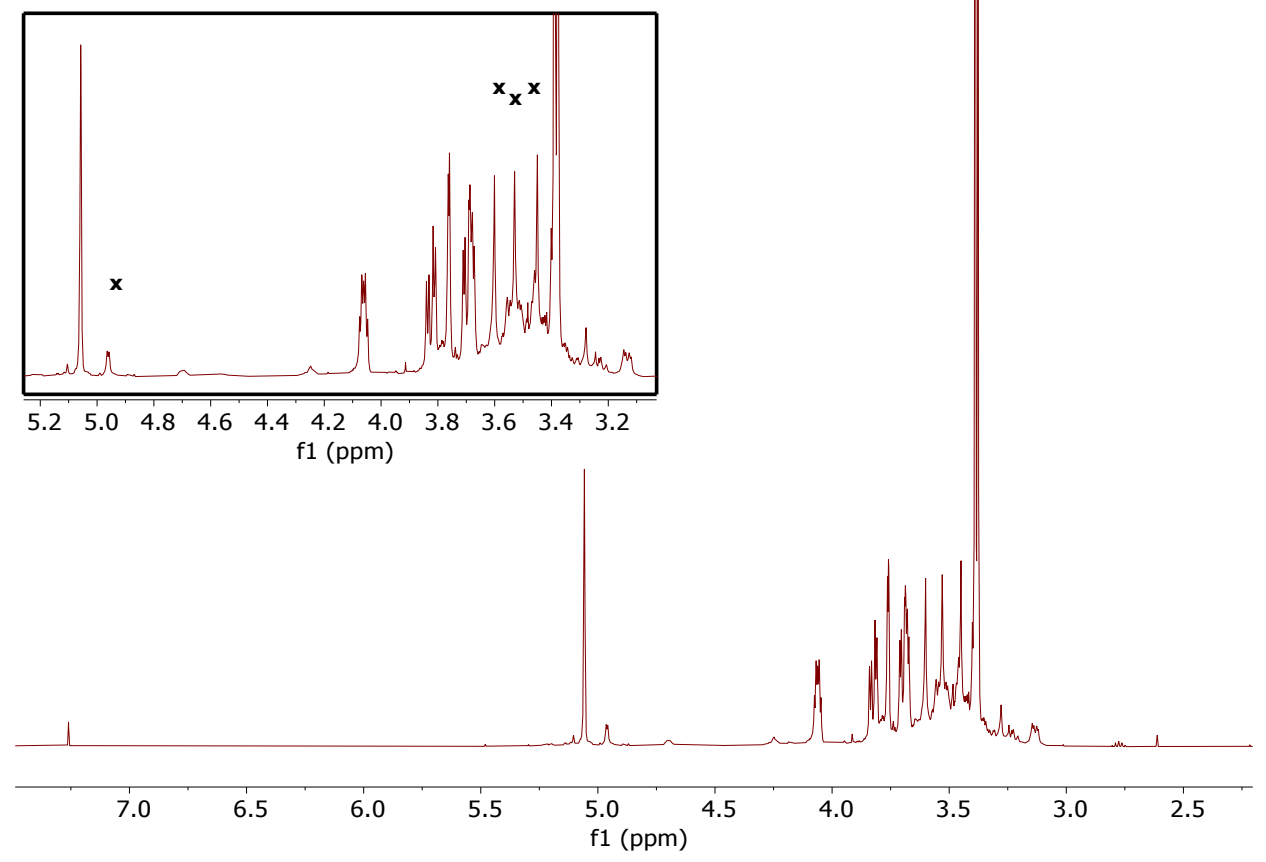

Figure S18: ${ }^{1} \mathrm{H}$ NMR spectrum of permethylated 1,5- $\alpha$-L-arabinan polysaccharide standard $(9.6 \mathrm{mg})$, showing an expanded region of $2.9-5.25 \mathrm{ppm}$, acquired in $600 \mu \mathrm{L} \mathrm{CDCl}_{3}$ with 16 scans on a Varian Inova $5 \mathrm{~mm}$ room temperature probe. There are impurity signals of 1,6- $\alpha$-glucan indicated with ' $\mathrm{x}$ ', which were assigned by chemical shift profiling with permethylated dextran standard.

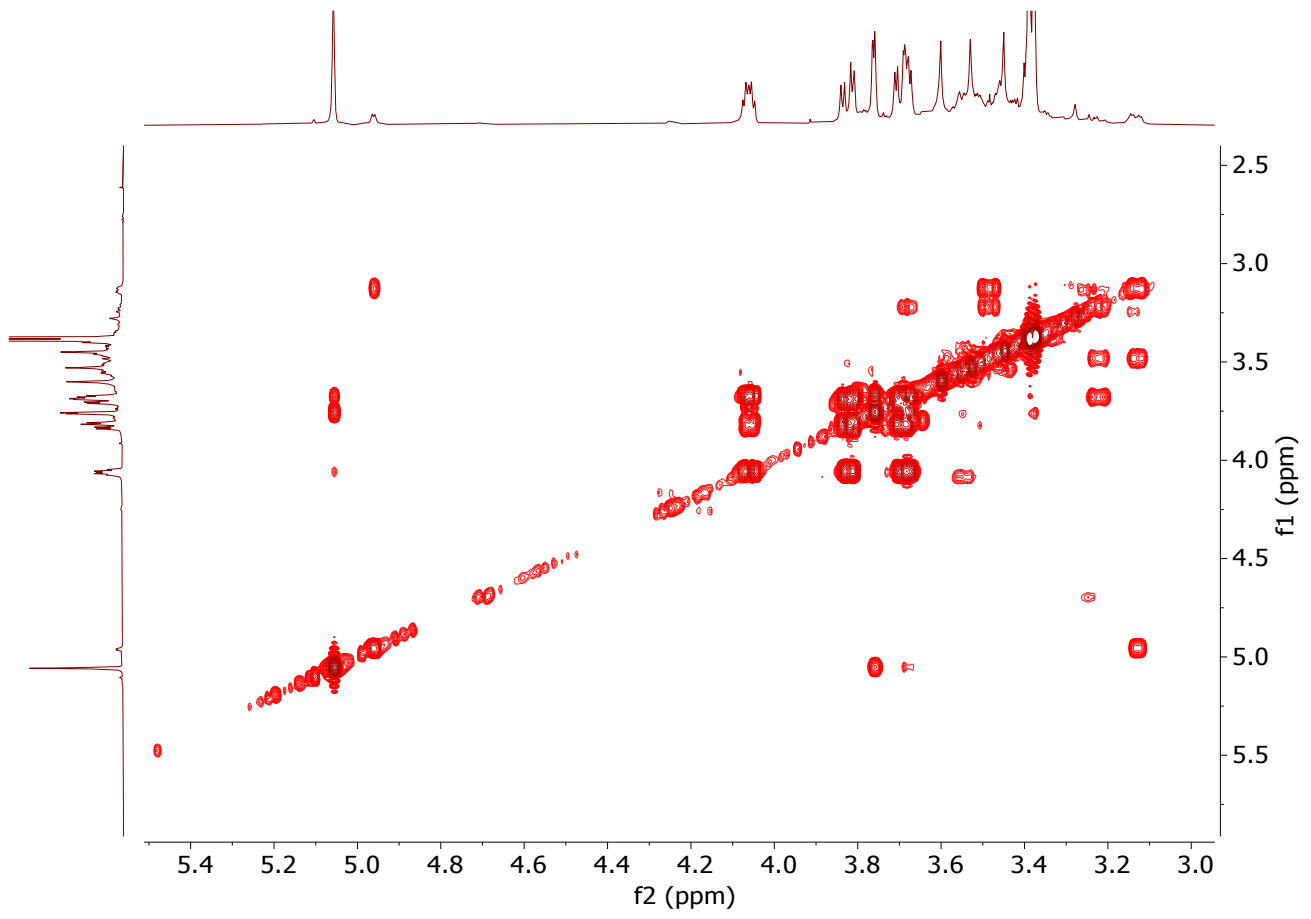

Figure S19: COSY NMR spectrum of permethylated 1,5- $\alpha$-L-arabinan polysaccharide standard (9.6 $\mathrm{mg})$ acquired in $(600 \mu \mathrm{L}) \mathrm{CDCl}_{3}$ with $1024 * 256(\mathrm{~F} 2 * \mathrm{~F} 1)$ data points and 10 scans per increment on a $5 \mathrm{~mm}$ Bruker TCI cryoprobe. 


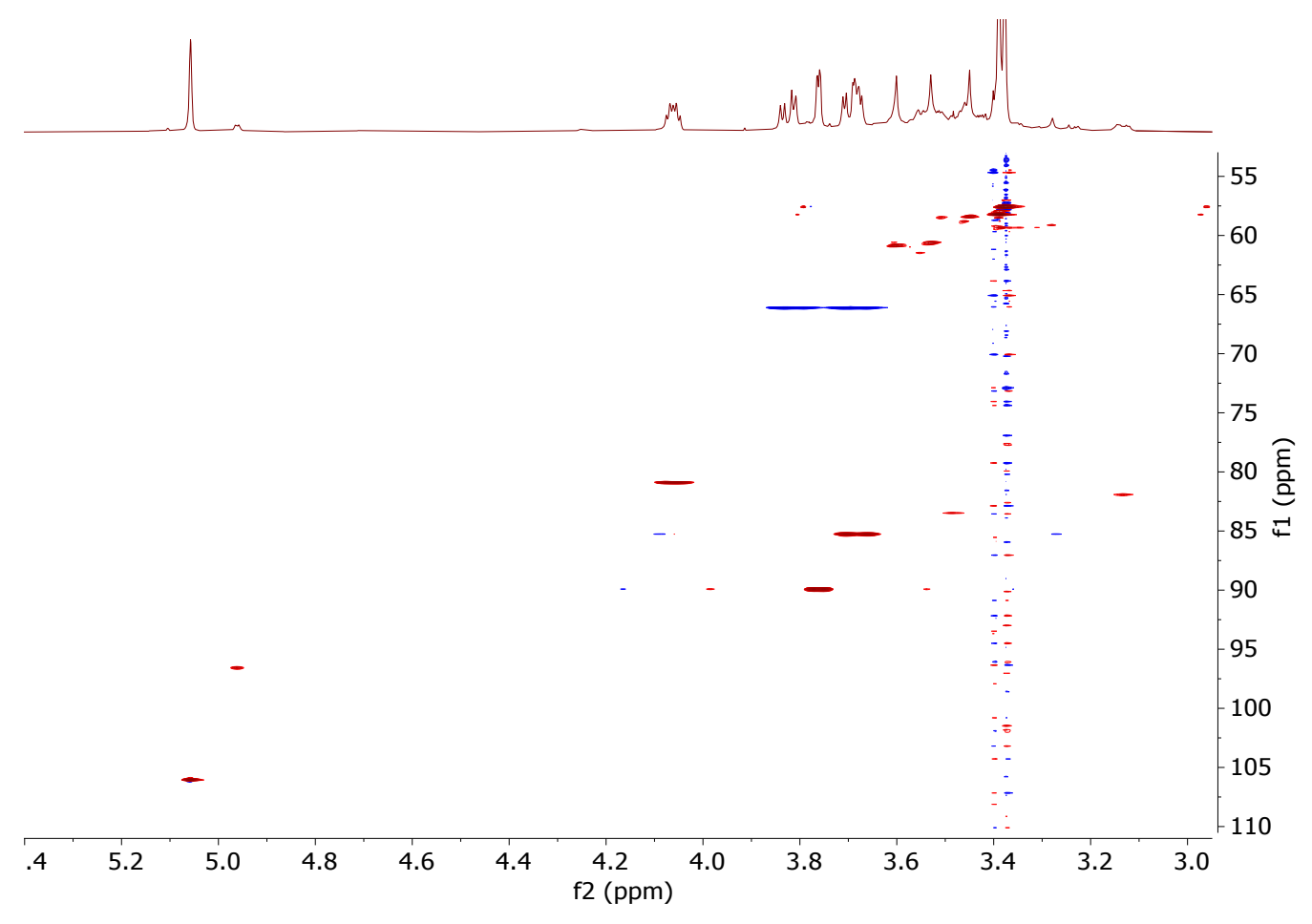

Figure S20: HSQC NMR spectrum of permethylated 1,5- $\alpha$-L-arabinan polysaccharide standard (9.6 $\mathrm{mg})$ acquired in $600 \mu \mathrm{L} \mathrm{CDCl}_{3}$ with $630 * 512(\mathrm{~F} 2 * \mathrm{~F} 1)$ data points and 4 scans per increment on a Varian Inova $5 \mathrm{~mm}$ room temperature probe.

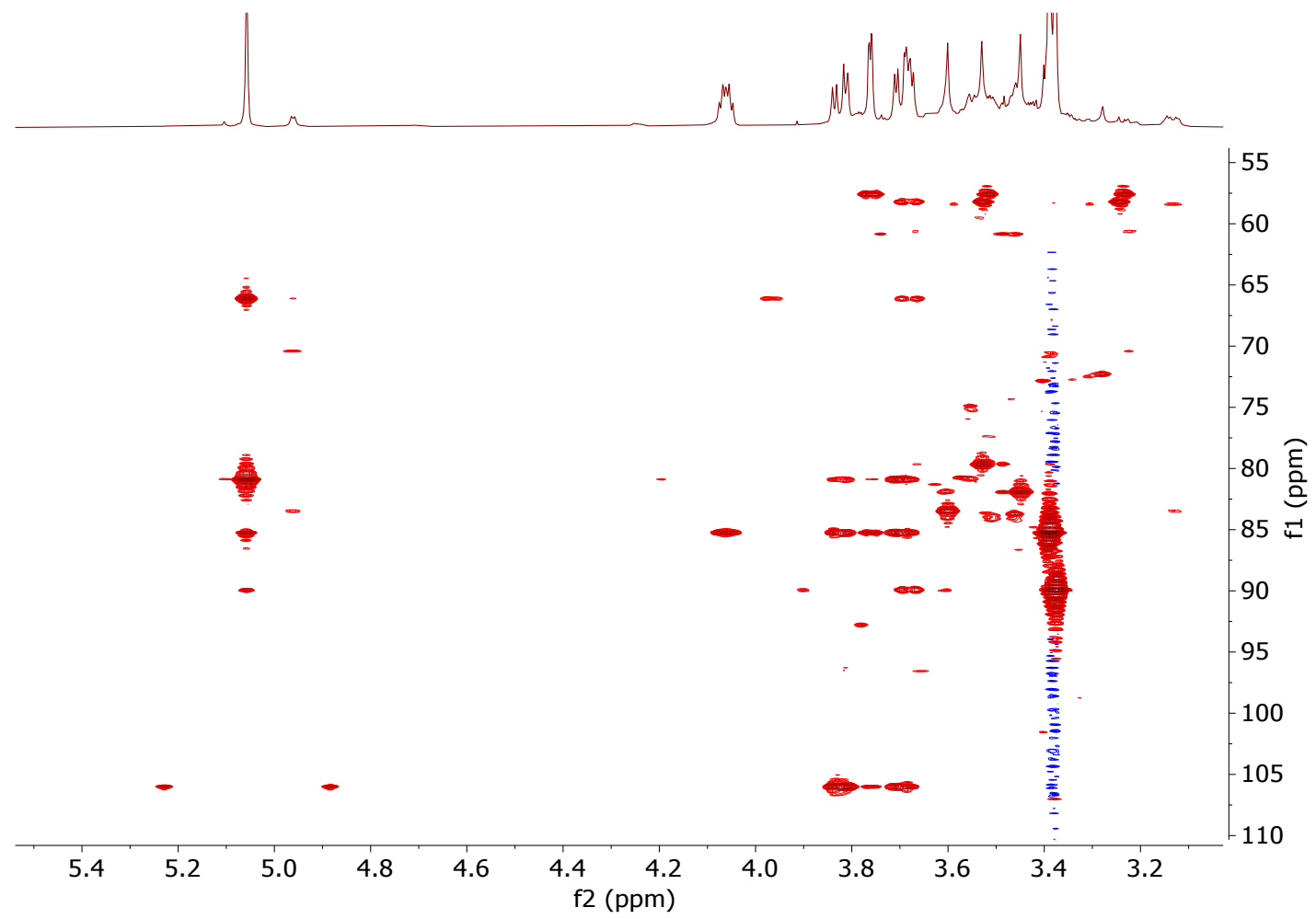

Figure S21: HMBC NMR spectrum of permethylated 1,5- $\alpha$-L-arabinan polysaccharide standard (9.6 $\mathrm{mg})$ acquired in $600 \mu \mathrm{L} \mathrm{CDCl}_{3}$ with $630 * 512(\mathrm{~F} 2 * \mathrm{~F} 1)$ data points and 24 scans per increment on a Varian Inova $5 \mathrm{~mm}$ room temperature probe. 


\section{Xylan NMR Data}

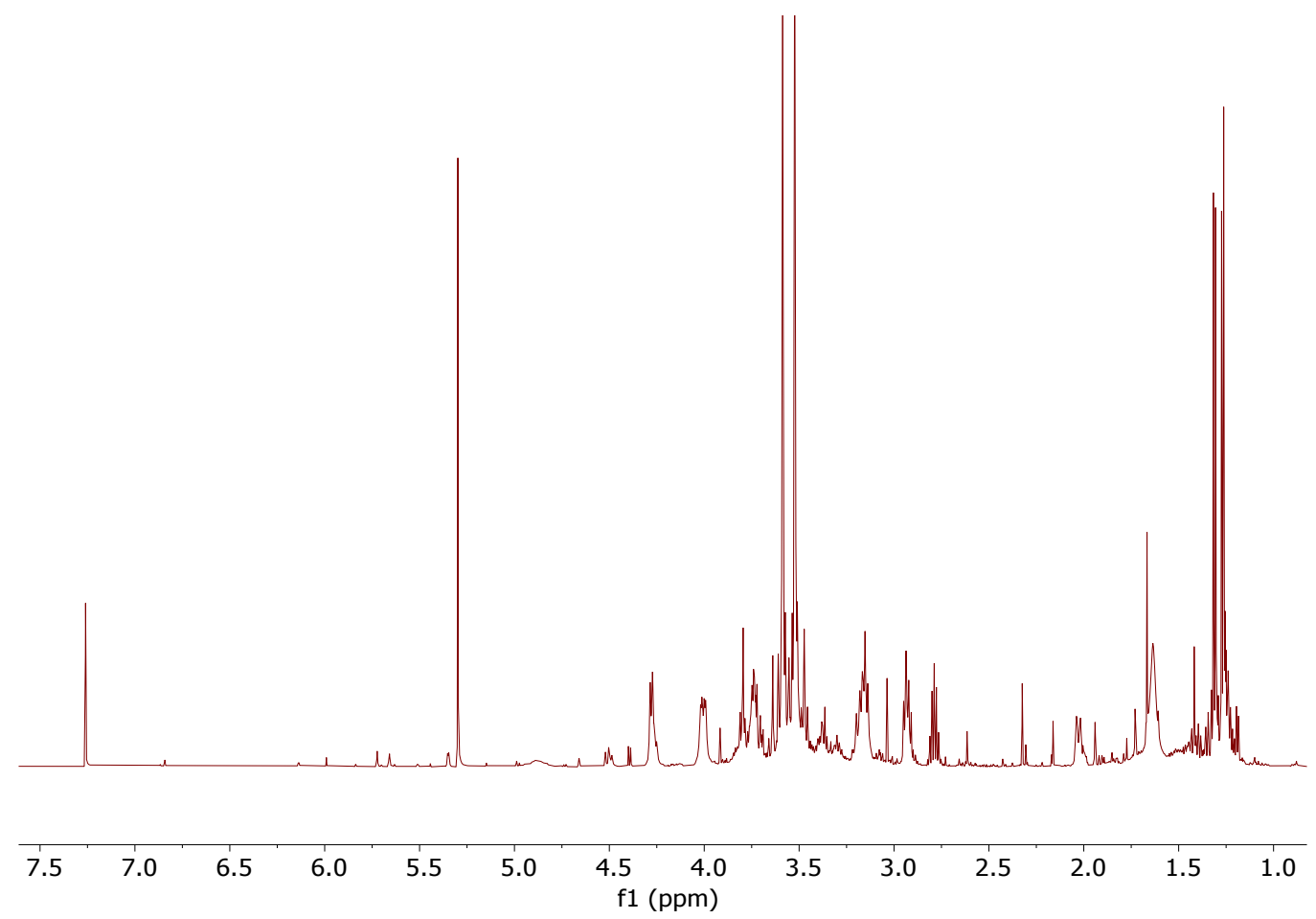

Figure S22: ${ }^{1} \mathrm{H}$ NMR spectrum of permethylated $1,4-\beta$-D-xylan polysaccharide standard acquired in $600 \mathrm{uL} \mathrm{CDCl}_{3}$ on a $5 \mathrm{~mm}$ Bruker TCI cryoprobe. Spectrum acquired with 4 scans. Evidence of significant unidentified impurities can be seen.

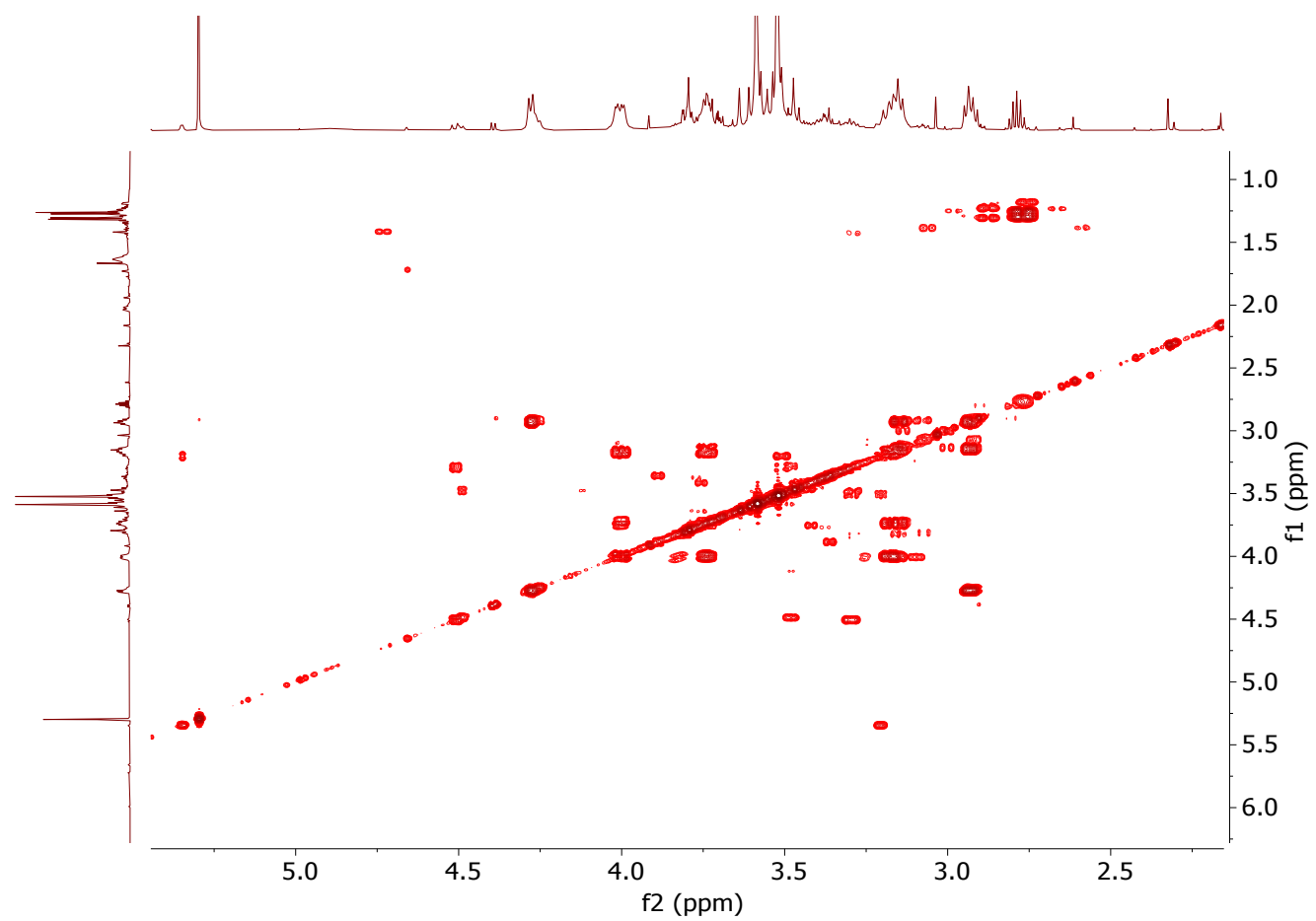

Figure S23: COSY NMR spectrum of permethylated 1,4- $\beta$-D-xylan polysaccharide standard acquired in $600 \mu \mathrm{L} \mathrm{CDCl} \mathrm{CD}_{3}$ with $1024 * 384(\mathrm{~F} 2 * \mathrm{~F} 1)$ data points and 4 scans per increment on a $5 \mathrm{~mm}$ Bruker TCI cryoprobe. 


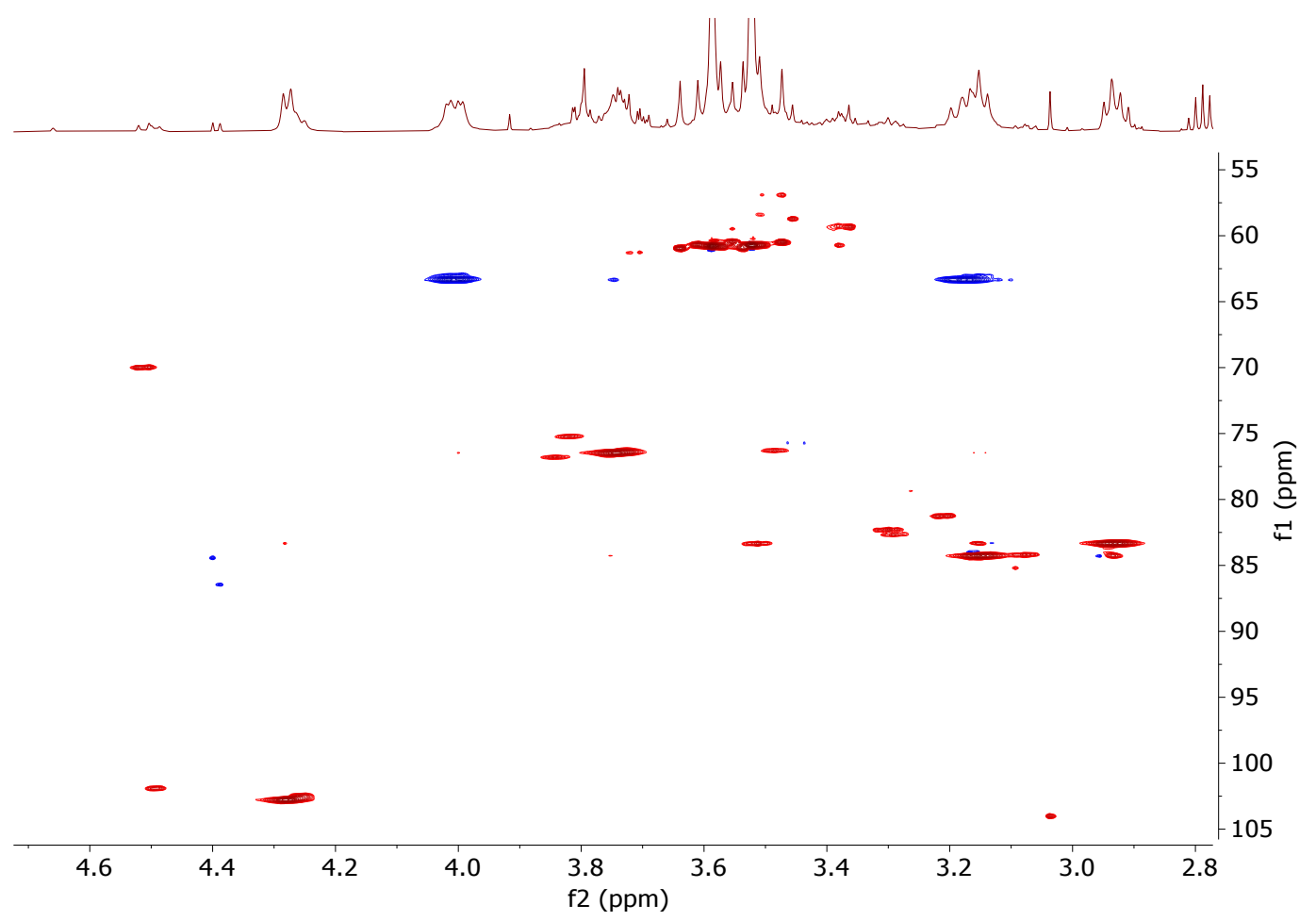

Figure S24: HSQC NMR spectrum of permethylated 1,4- $\beta$-D-xylan polysaccharide standard acquired in $600 \mu \mathrm{L} \mathrm{CDCl} \mathrm{Cl}_{3}$ with $768 * 562(\mathrm{~F} 2 * \mathrm{~F} 1)$ data points and 4 scans per increment on a $5 \mathrm{~mm}$ Bruker TCI cryoprobe.

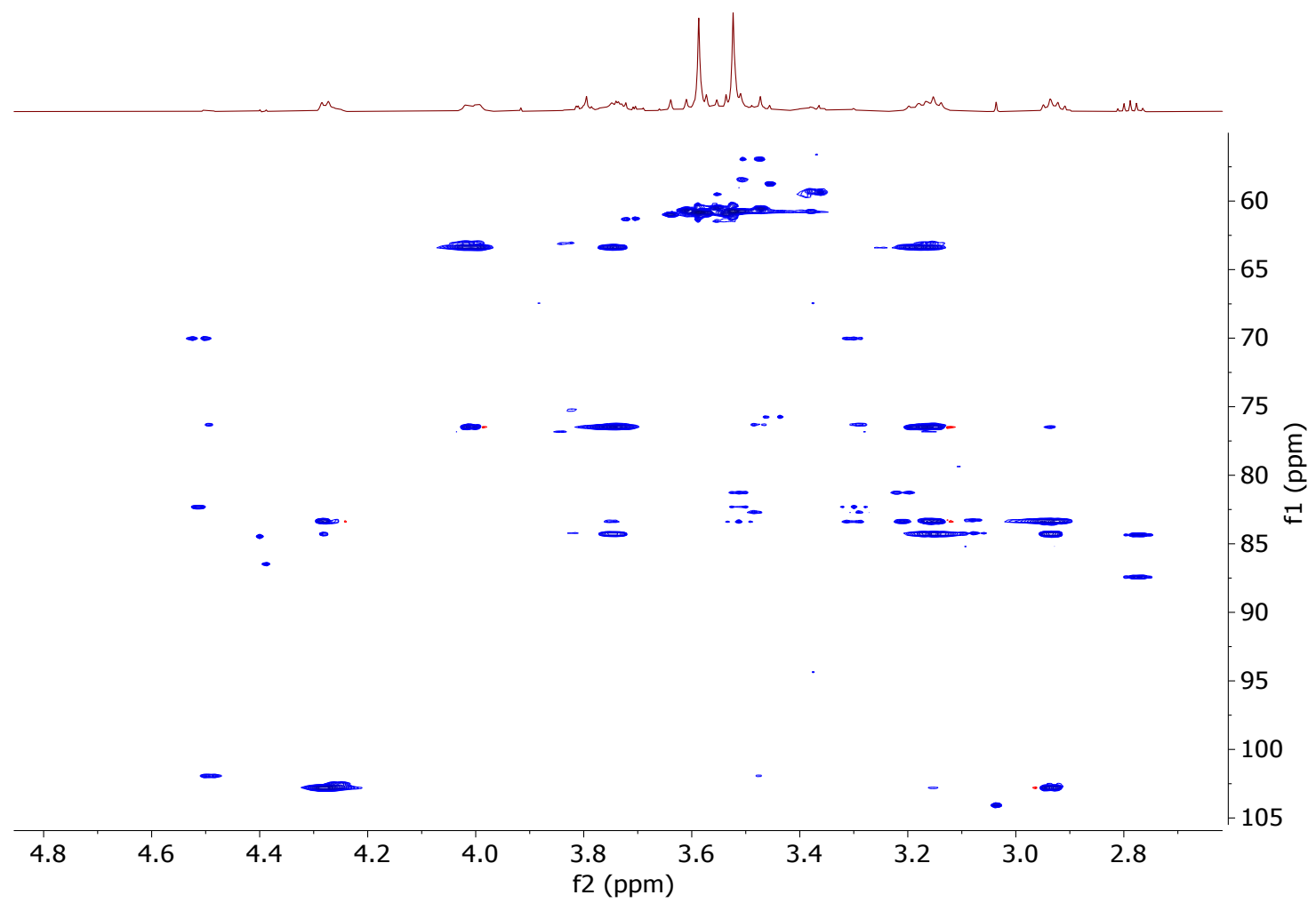

Figure S25: HSQC-TOCSY NMR spectrum of permethylated 1,4- $\beta$-D-xylan polysaccharide standard acquired in $600 \mu \mathrm{L} \mathrm{CDCl}_{3}$ with $768 * 768(\mathrm{~F} 2 * \mathrm{~F} 1)$ data points, 8 scans per increment and $18 \mathrm{~ms}$ mixing time on a $5 \mathrm{~mm}$ Bruker TCI cryoprobe. 


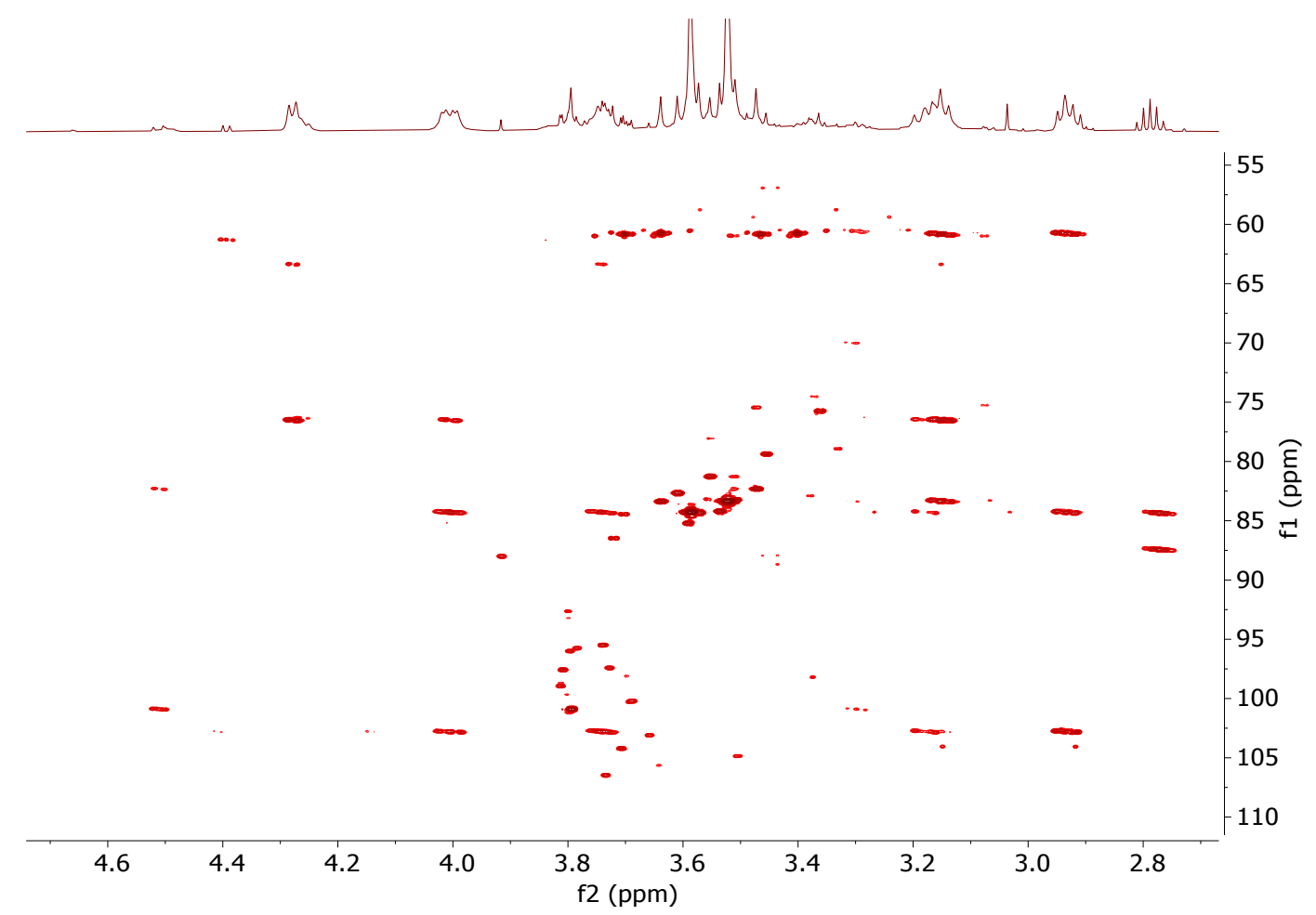

Figure S26: HMBC NMR spectrum of permethylated 1,4- $\beta$-D-xylan polysaccharide standard acquired in $600 \mu \mathrm{L} \mathrm{CDCl}_{3}$ with $1536 * 192(\mathrm{~F} 2 * \mathrm{~F} 1)$ data points and 40 scans per increment on a $5 \mathrm{~mm}$ Bruker TCI cryoprobe.

\section{Mannan NMR Data}

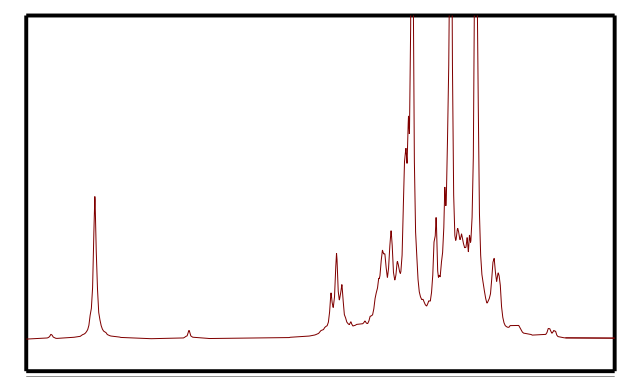

\begin{tabular}{llllllllllll}
\hline 4.8 & 4.6 & 4.4 & 4.2 & 4.0 & 3.8 & 3.6 & 3.4 & 3.2 & 3.0
\end{tabular}

f1 (ppm)
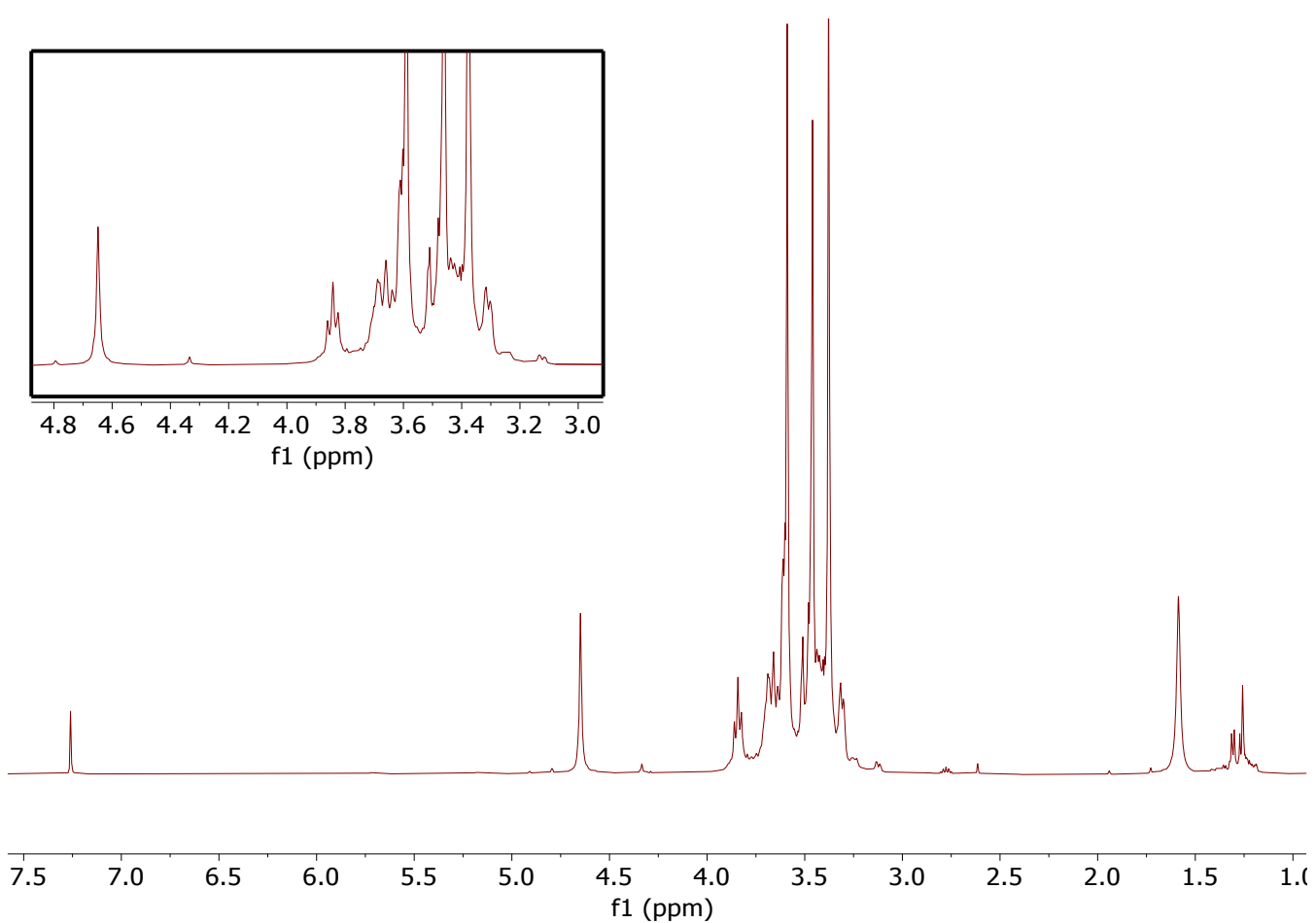

Figure S27: ${ }^{1} \mathrm{H}$ NMR spectrum of permethylated $1,4-\beta$-D-mannan $(9.1 \mathrm{mg})$ polysaccharide standard, showing an expanded region of $2.9-4.9 \mathrm{ppm}$, acquired in $600 \mu \mathrm{L} \mathrm{CDCl}_{3}$ with 32 scans on a Varian Inova room temperature $5 \mathrm{~mm}$ probe. 


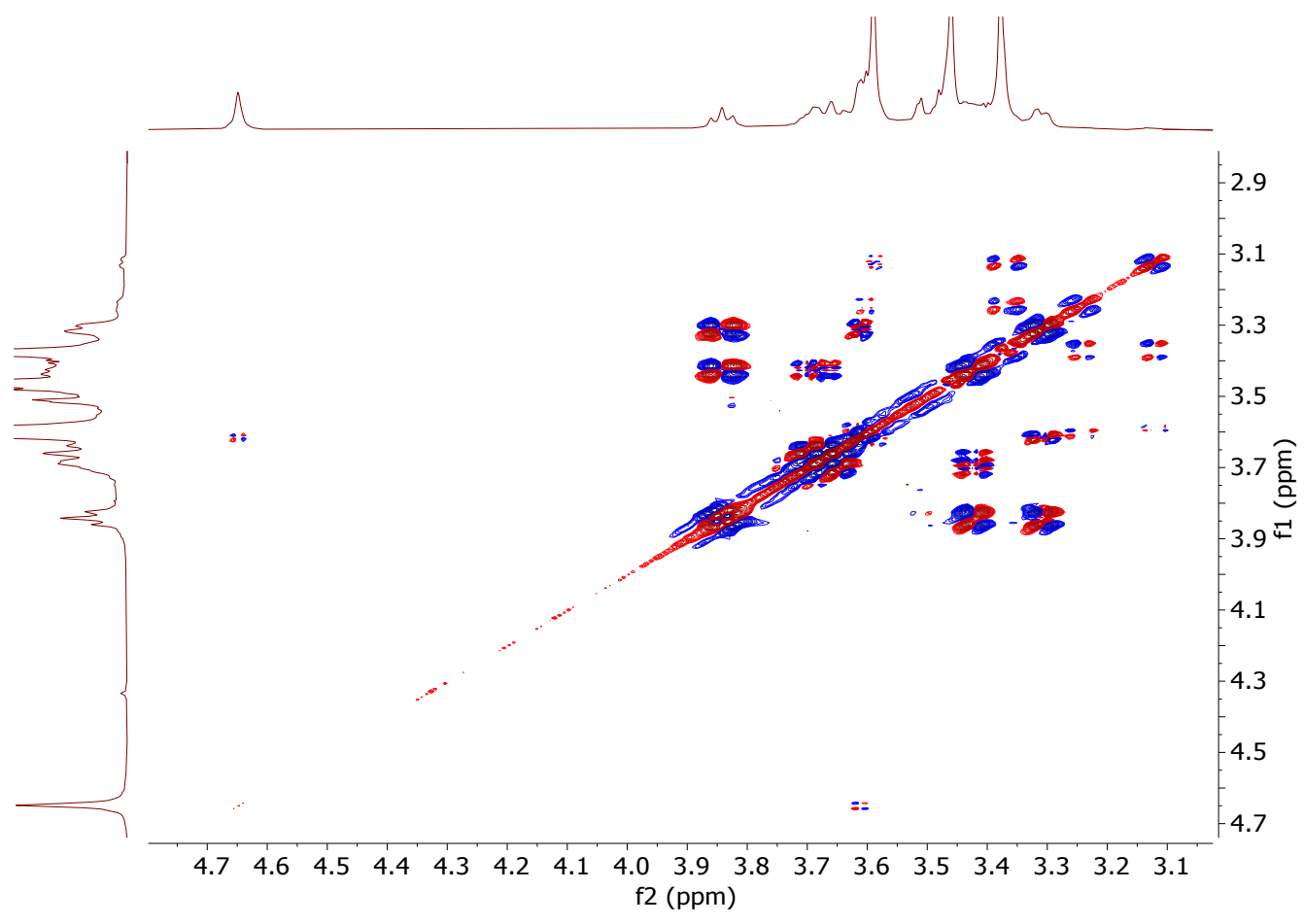

Figure S28: DQFCOSY NMR spectrum of permethylated 1,4- $\beta$-D-mannan polysaccharide standard $(9.1 \mathrm{mg})$ acquired in $600 \mu \mathrm{L} \mathrm{CDCl}_{3}$ with $657 * 512(\mathrm{~F} 2 * \mathrm{~F} 1)$ data points and 4 scans per increment on a Varian Inova room temperature $5 \mathrm{~mm}$ probe.

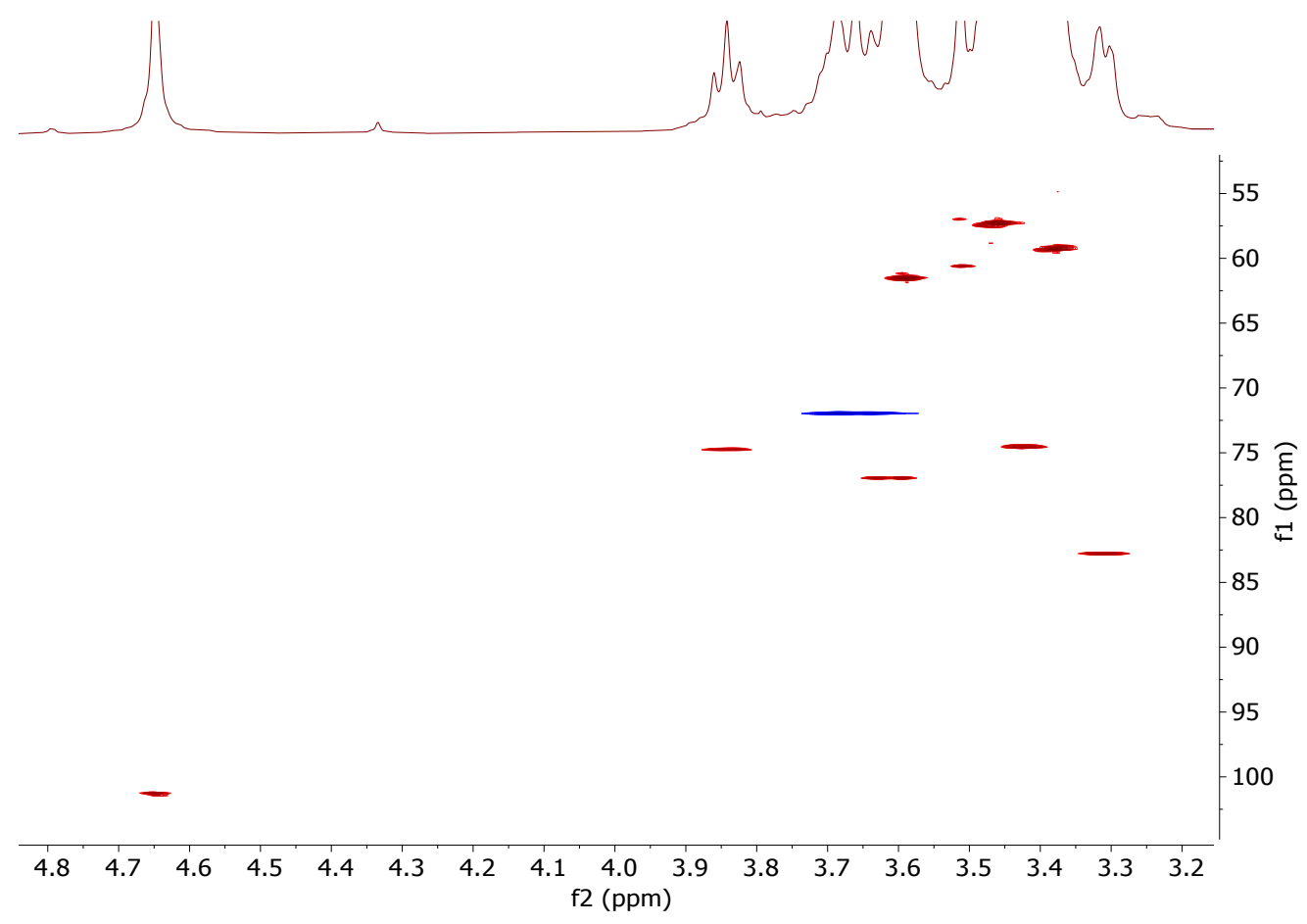

Figure S29: HSQC NMR spectrum of permethylated 1,4- $\beta$-D-mannan polysaccharide standard (9.1 $\mathrm{mg})$ acquired in $600 \mu \mathrm{L} \mathrm{CDCl} \mathrm{CD}_{3}$ with $630 * 512(\mathrm{~F} 2 * \mathrm{~F} 1)$ data points and 8 scans per increment on a Varian Inova room temperature $5 \mathrm{~mm}$ probe. 


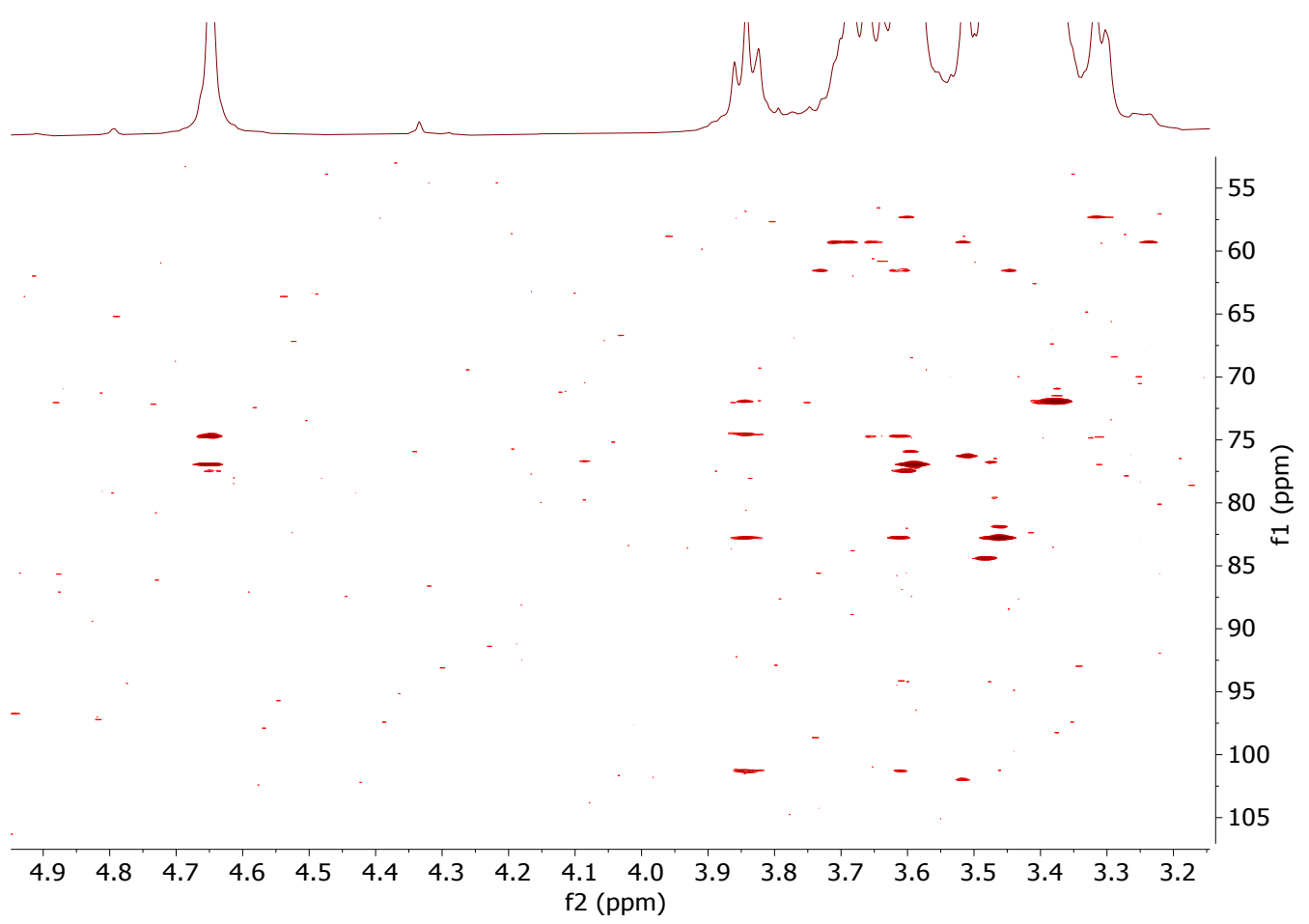

Figure S30: HMBC NMR spectrum of permethylated 1,4- $\beta$-D-mannan polysaccharide standard (9.1 $\mathrm{mg})$ acquired in $600 \mu \mathrm{L} \mathrm{CDCl}{ }_{3}$ with $1024 * 512(\mathrm{~F} 2 * \mathrm{~F} 1)$ data points and 12 scans per increment on a Varian Inova room temperature $5 \mathrm{~mm}$ probe.

\section{Cellulose NMR Data}
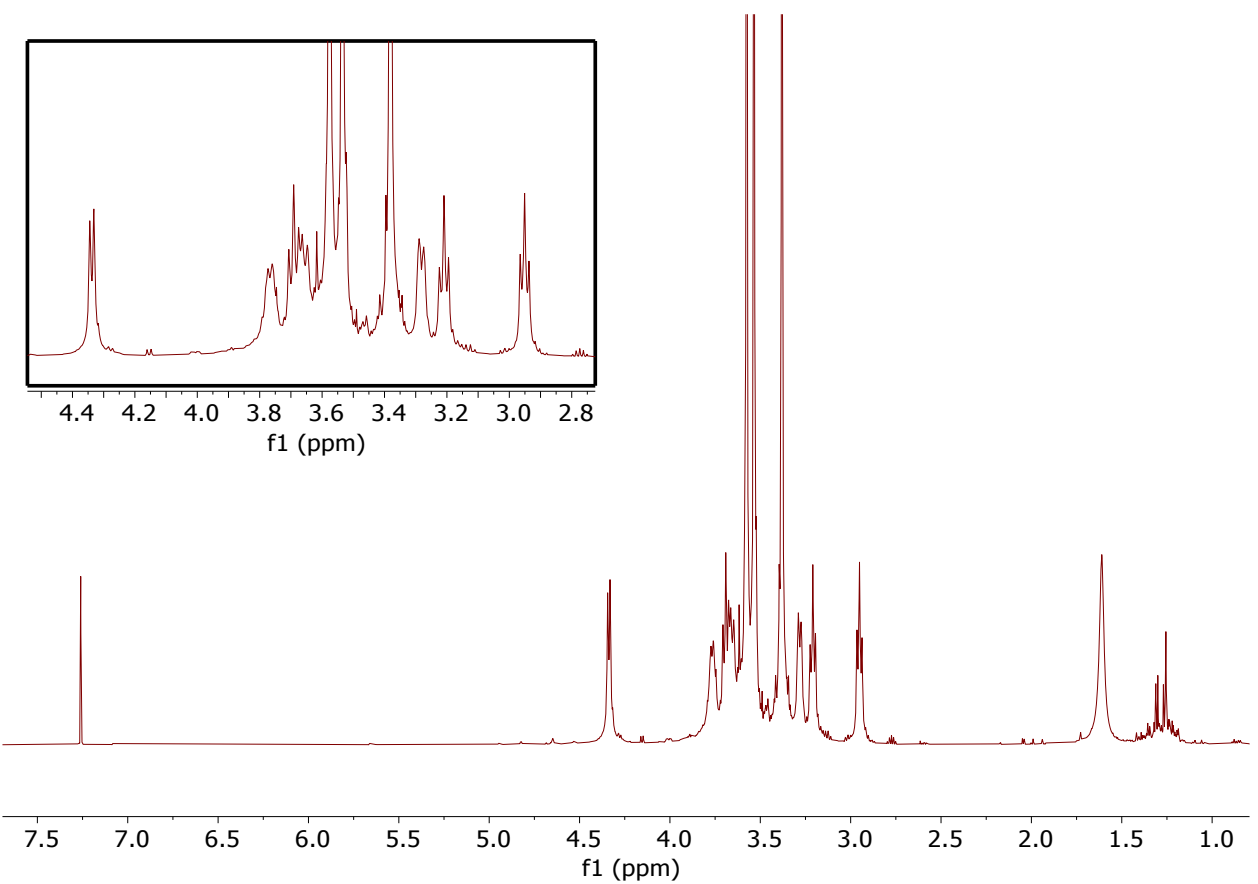

Figure S31: ${ }^{1} \mathrm{H}$ NMR spectrum of permethylated 1,4- $\beta$-D-glucan (cellulose) polysaccharide standard $(\sim 5.0 \mathrm{mg})$, showing an expanded region of $2.75-4.60 \mathrm{ppm}$, acquired in $600 \mu \mathrm{L} \mathrm{CDCl}_{3}$ with 8 scans on a $5 \mathrm{~mm}$ VNMRS HNC cryoprobe. 


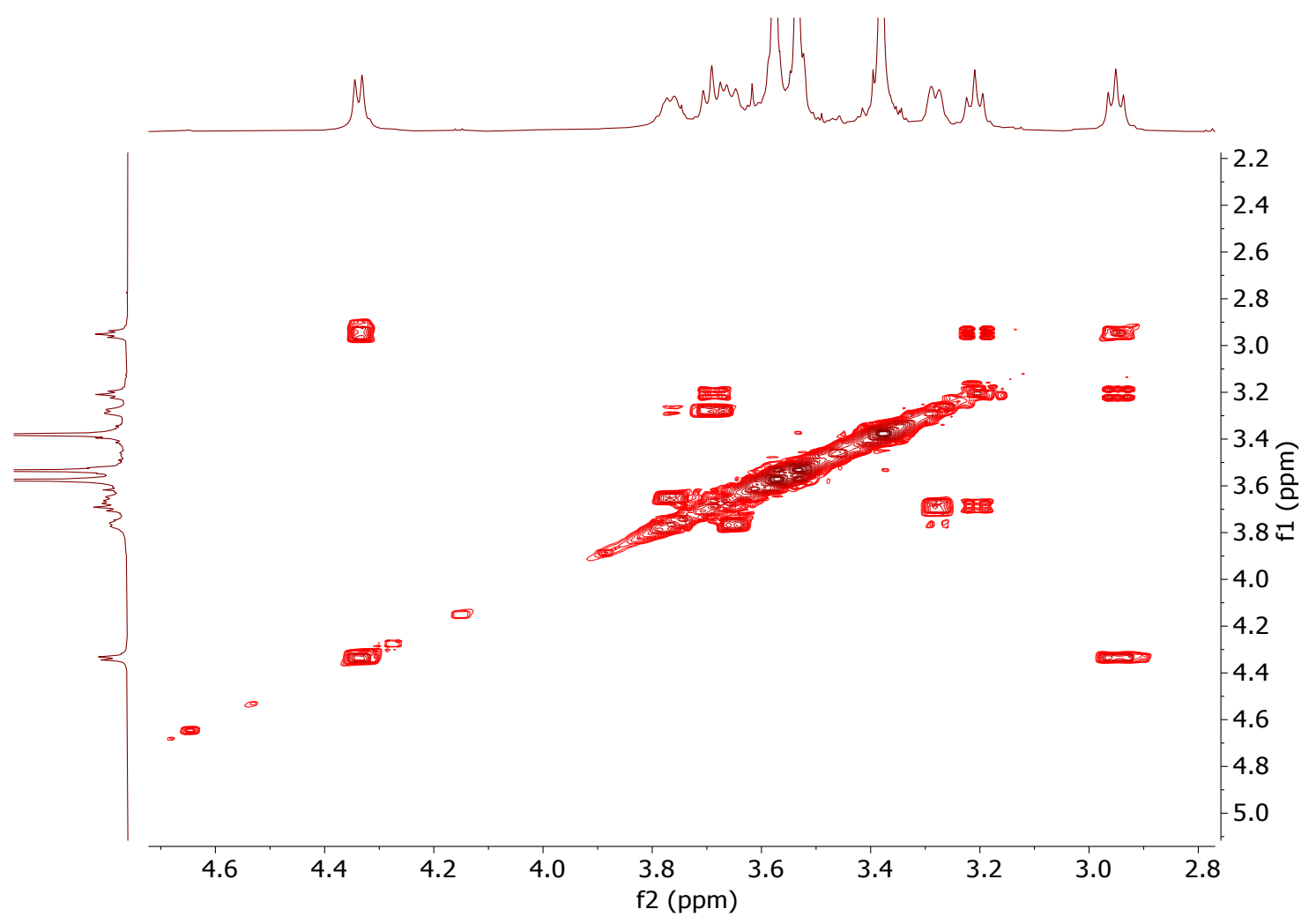

Figure S32: COSY NMR spectrum of permethylated 1,4- $\beta$-D-glucan (cellulose) polysaccharide standard $(\sim 5.0 \mathrm{mg})$, acquired in $600 \mu \mathrm{L} \mathrm{CDCl}_{3}$ with $951 * 256(\mathrm{~F} 2 * \mathrm{~F} 1)$ data points with 8 scans per increment on a $5 \mathrm{~mm}$ VNMRS HNC cryoprobe.

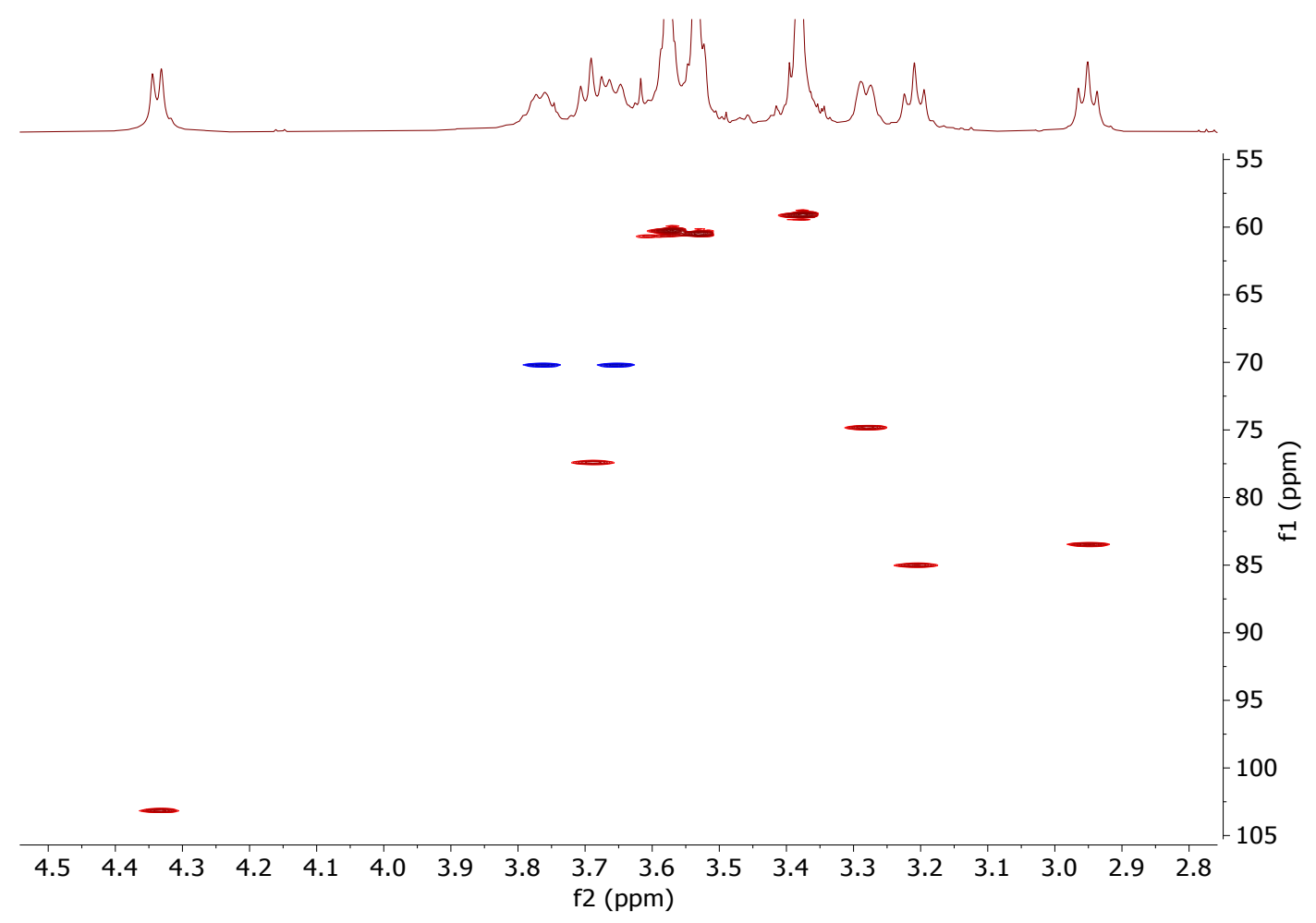

Figure S33: HSQC NMR spectrum of permethylated 1,4- $\beta$-D-glucan (cellulose) polysaccharide standard $(\sim 5.0 \mathrm{mg})$, acquired in $600 \mu \mathrm{L} \mathrm{CDCl}_{3}$ with $817 * 256(\mathrm{~F} 2 * \mathrm{~F} 1)$ data points with 20 scans per increment on a $5 \mathrm{~mm}$ VNMRS HNC cryoprobe. 


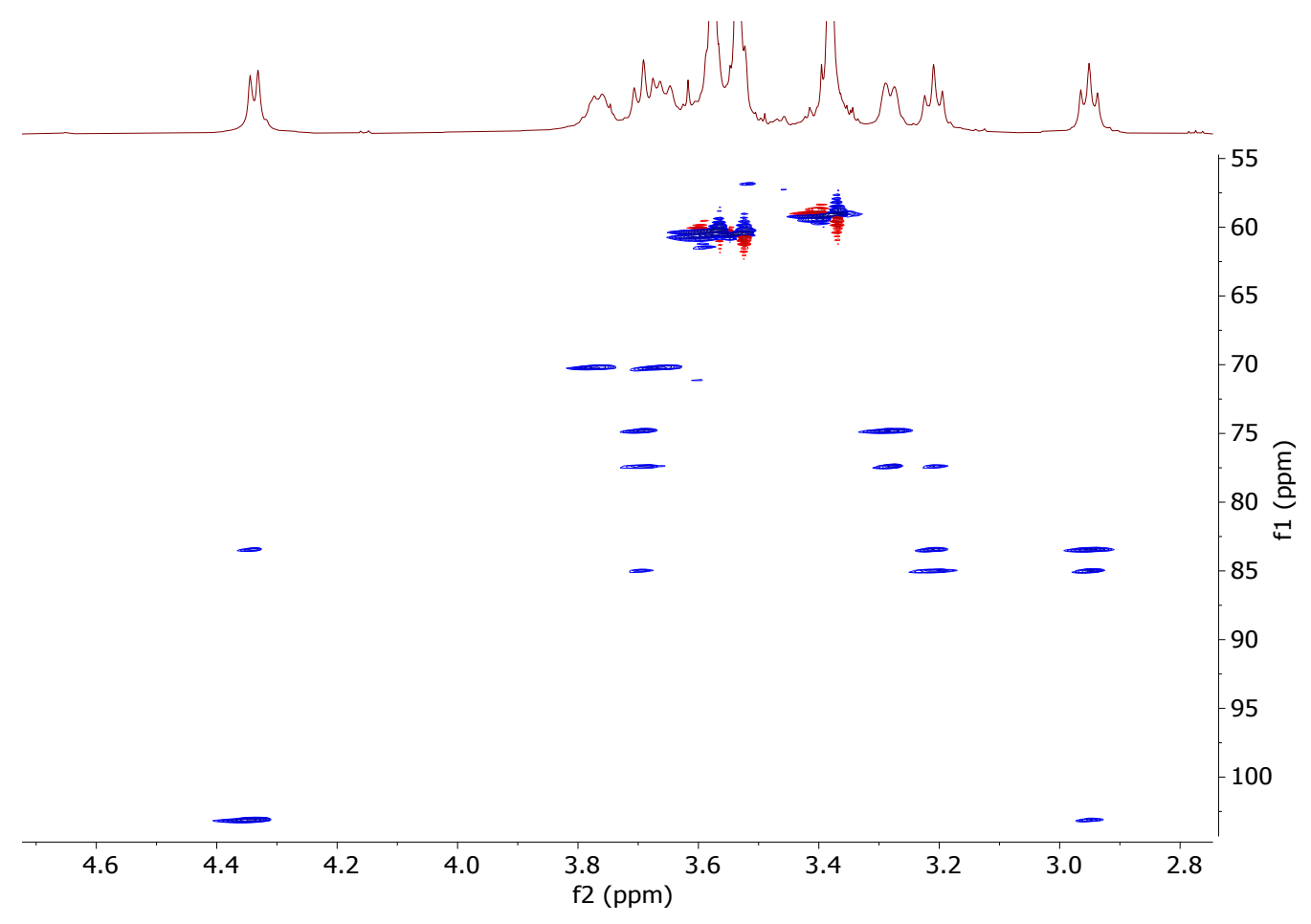

Figure S34: HSQC-TOCSY NMR spectrum of permethylated 1,4- $\beta$-D-glucan (cellulose) polysaccharide standard $(\sim 5.0 \mathrm{mg})$, acquired in $600 \mu \mathrm{L} \mathrm{CDCl}_{3}$ with $817 * 256(\mathrm{~F} 2 * \mathrm{~F} 1)$ data points with 36 scans per increment on a $5 \mathrm{~mm}$ VNMRS HNC cryoprobe, with $18 \mathrm{~ms}$ mixing time.

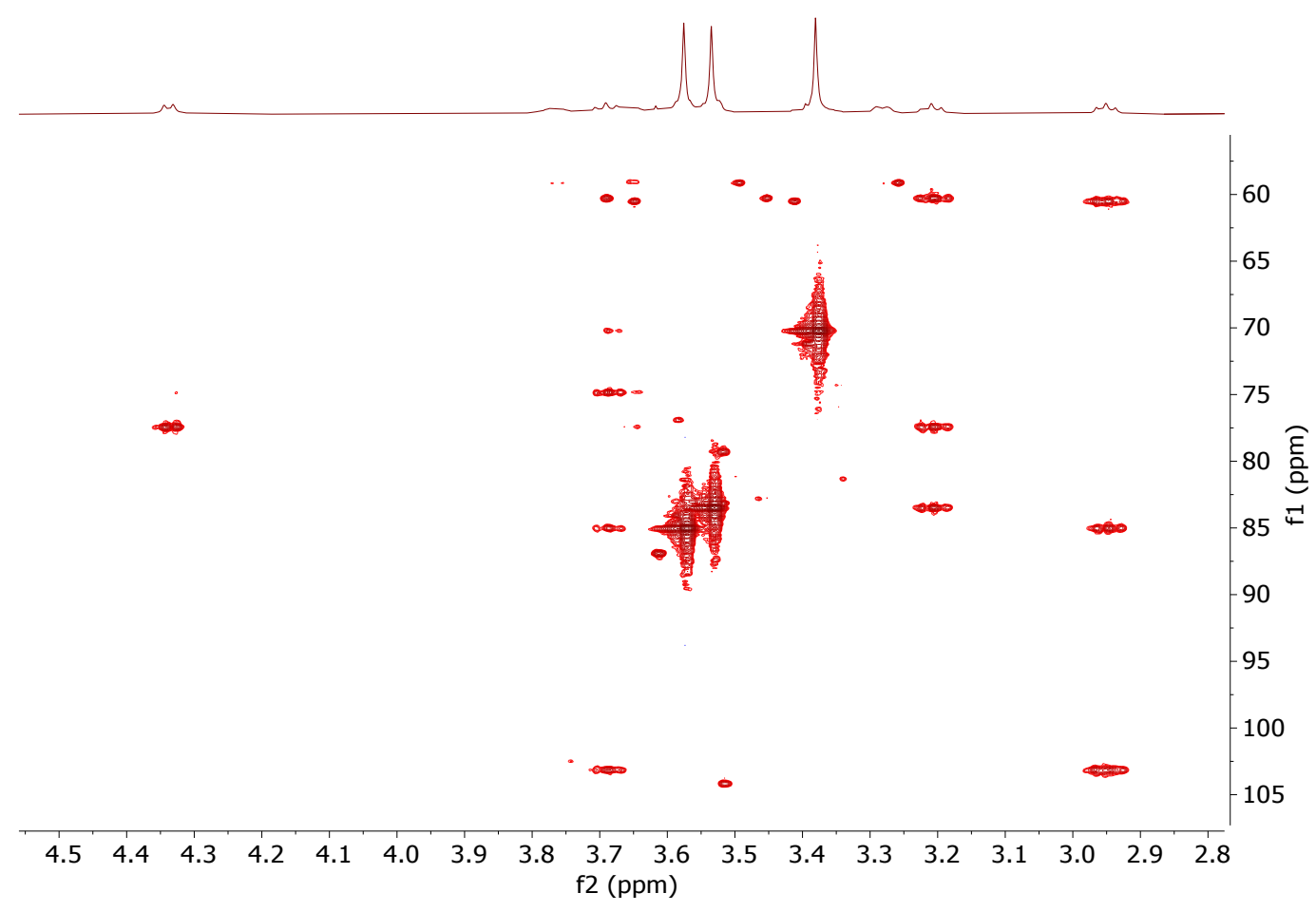

Figure S35: HMBC NMR spectrum of permethylated 1,4- $\beta$-D-glucan (cellulose) polysaccharide standard $(\sim 5.0 \mathrm{mg})$, acquired in $600 \mu \mathrm{L} \mathrm{CDCl}{ }_{3}$ with $1256 * 256(\mathrm{~F} 2 * \mathrm{~F} 1)$ data points with 24 scans per increment on a $5 \mathrm{~mm}$ VNMRS HNC cryoprobe. 


\section{Starch NMR Data}

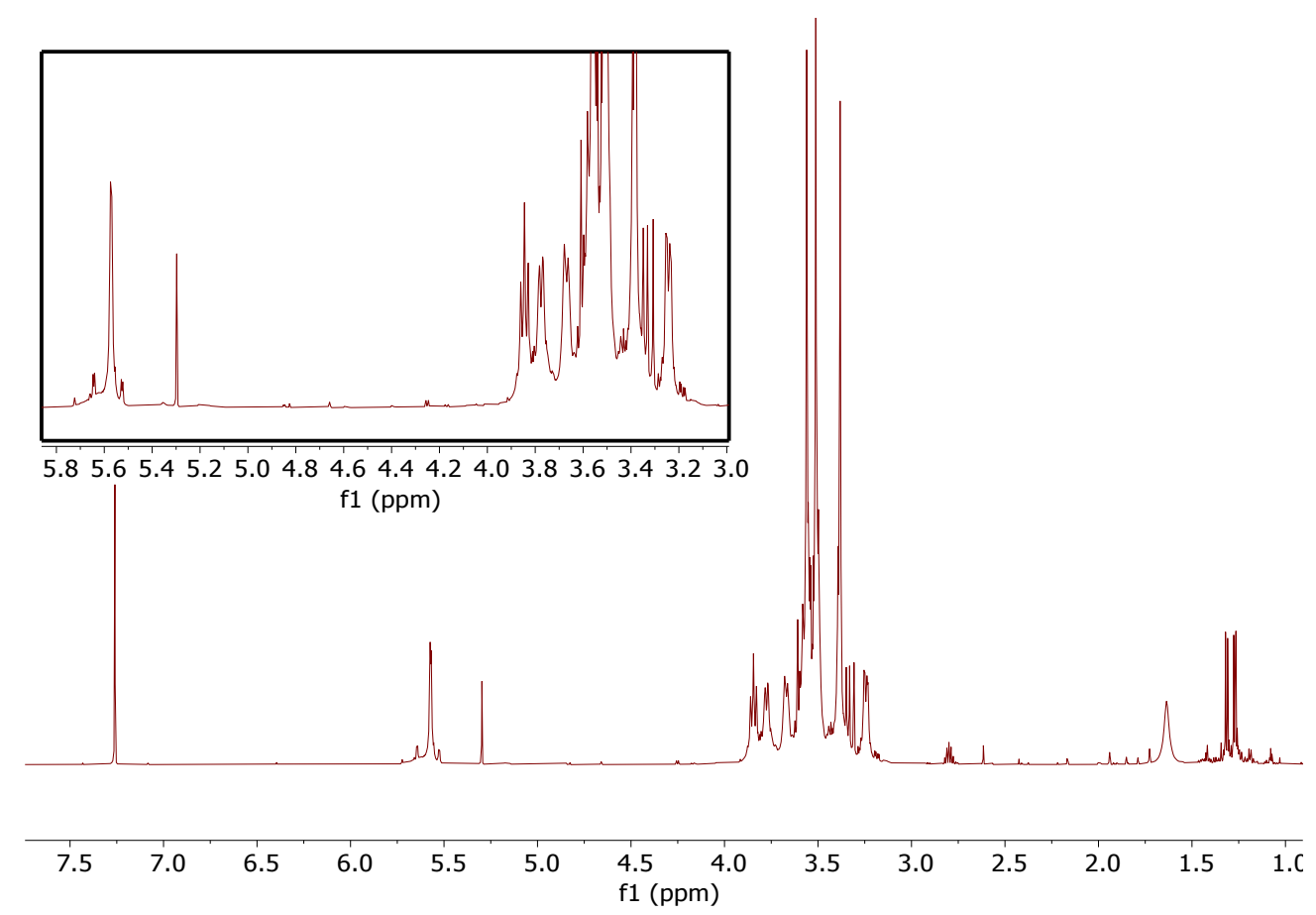

Figure S36: ${ }^{1} \mathrm{H}$ NMR spectrum of permethylated $1,4-\alpha-\mathrm{D}$-glucan (starch) polysaccharide standard (5.0 $\mathrm{mg}$ ), showing an expanded region of $3.00-5.90 \mathrm{ppm}$, acquired in $600 \mu \mathrm{L} \mathrm{CDCl}_{3}$ with 4 scans on a Bruker $600 \mathrm{MHz}$ spectrometer equipped with $5 \mathrm{~mm}$ TCI cryoprobe. There are minor residue peak that have not been fully analysed.

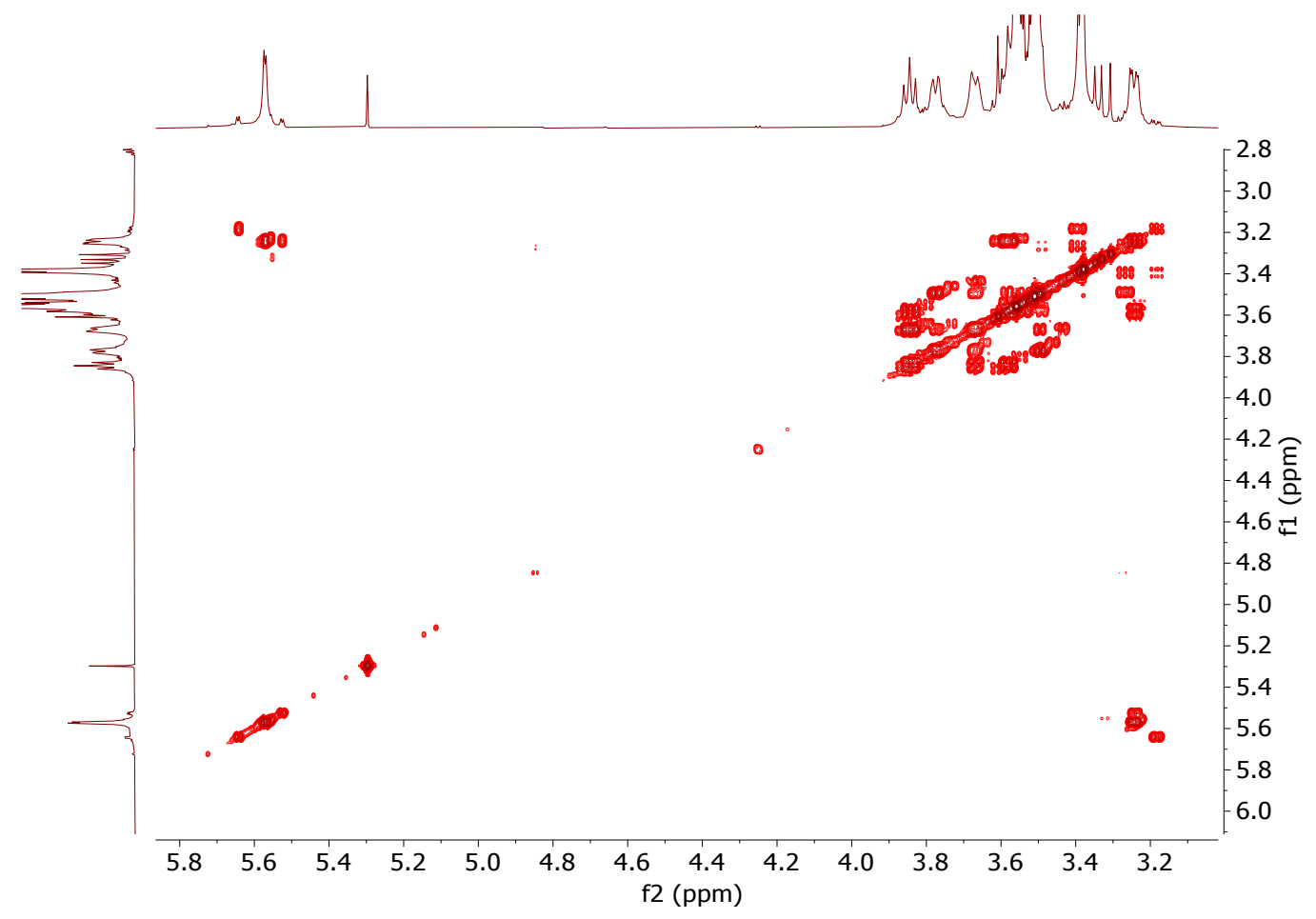

Figure S37: COSY NMR spectrum of permethylated 1,4- $\alpha$-D-glucan (starch) polysaccharide standard $(5.0 \mathrm{mg})$ acquired in $600 \mu \mathrm{L} \mathrm{CDCl}_{3}$ with $1280 * 512(\mathrm{~F} 2 * \mathrm{~F} 1)$ data points with 8 scans per increment on a Bruker $600 \mathrm{MHz}$ spectrometer equipped with $5 \mathrm{~mm}$ TCI cryoprobe. 


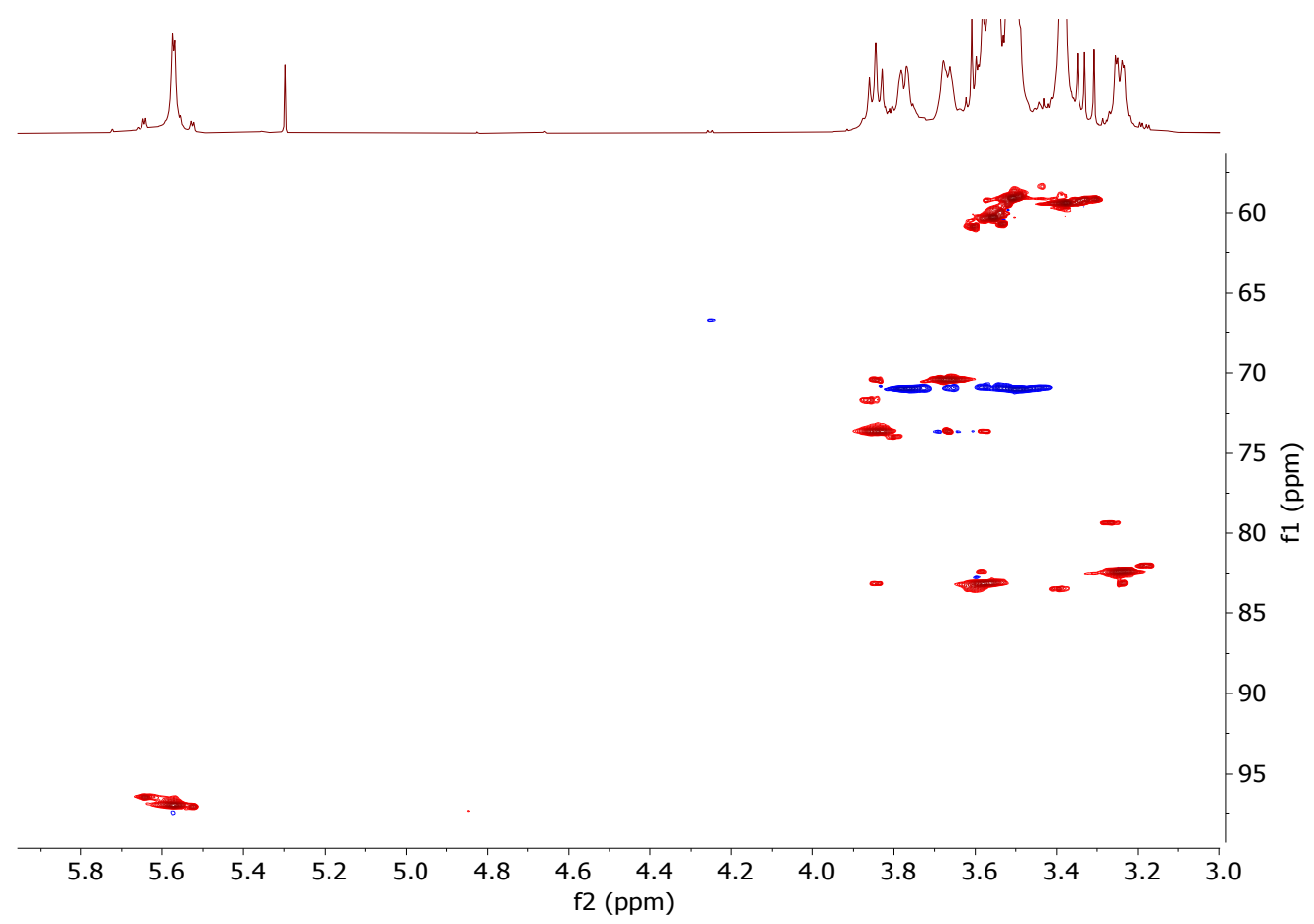

Figure S38: HSQC NMR spectrum of permethylated 1,4- $\alpha$-D-glucan (starch) polysaccharide standard $(5.0 \mathrm{mg})$, acquired in $600 \mu \mathrm{L} \mathrm{CDCl}{ }_{3}$ with $768 * 256(\mathrm{~F} 2 * \mathrm{~F} 1)$ data points with 32 scans per increment on a Bruker $600 \mathrm{MHz}$ spectrometer equipped with $5 \mathrm{~mm}$ TCI cryoprobe.

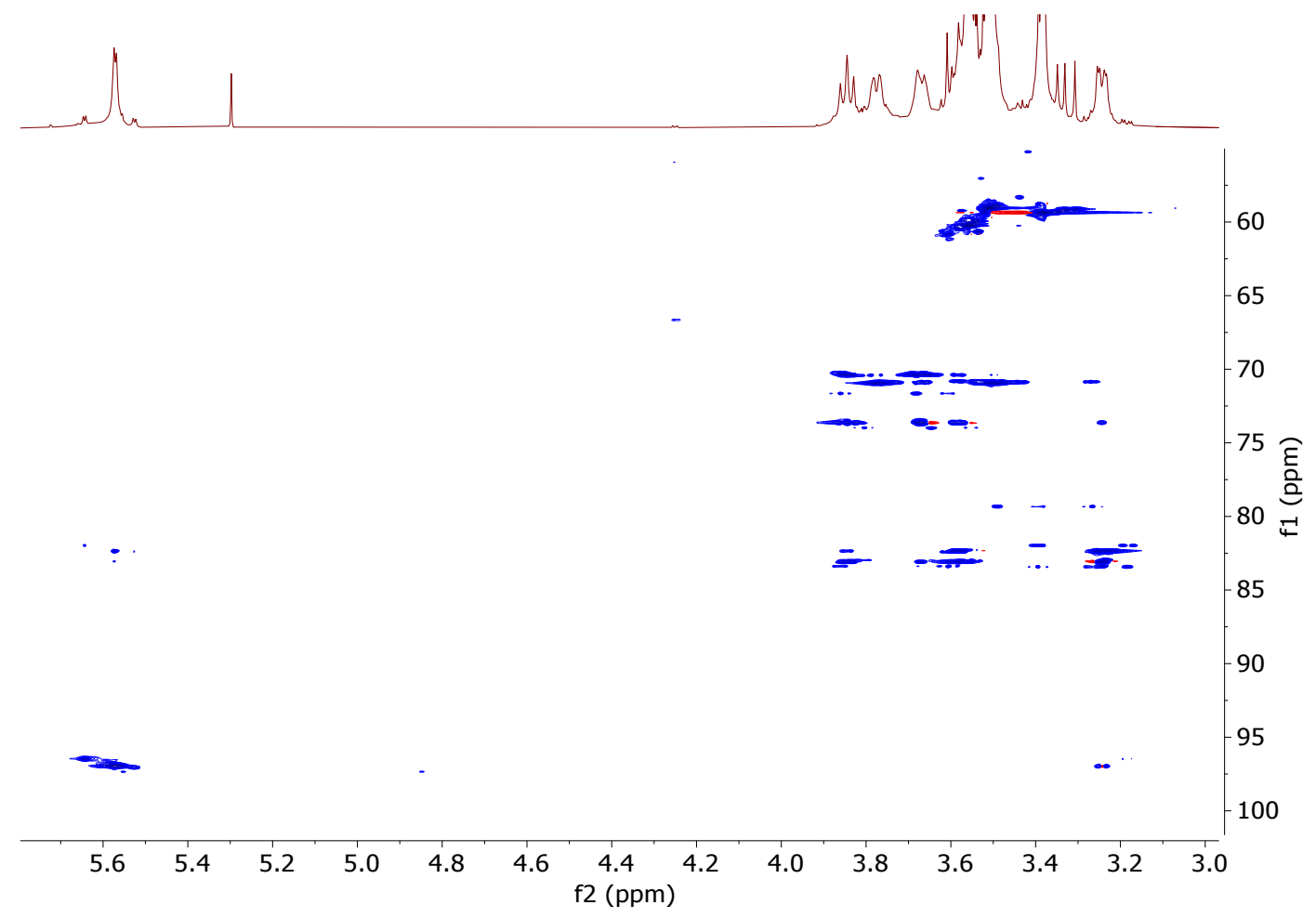

Figure S39: HSQC-TOCSY NMR spectrum of permethylated 1,4- $\alpha$-D-glucan (starch) polysaccharide standard $(5.0 \mathrm{mg})$, acquired in $600 \mu \mathrm{L} \mathrm{CDCl}_{3}$ with $819 * 768(\mathrm{~F} 2 * \mathrm{~F} 1)$ data points with 16 scans per increment on a Bruker $600 \mathrm{MHz}$ spectrometer equipped with $5 \mathrm{~mm}$ TCI cryoprobe. 


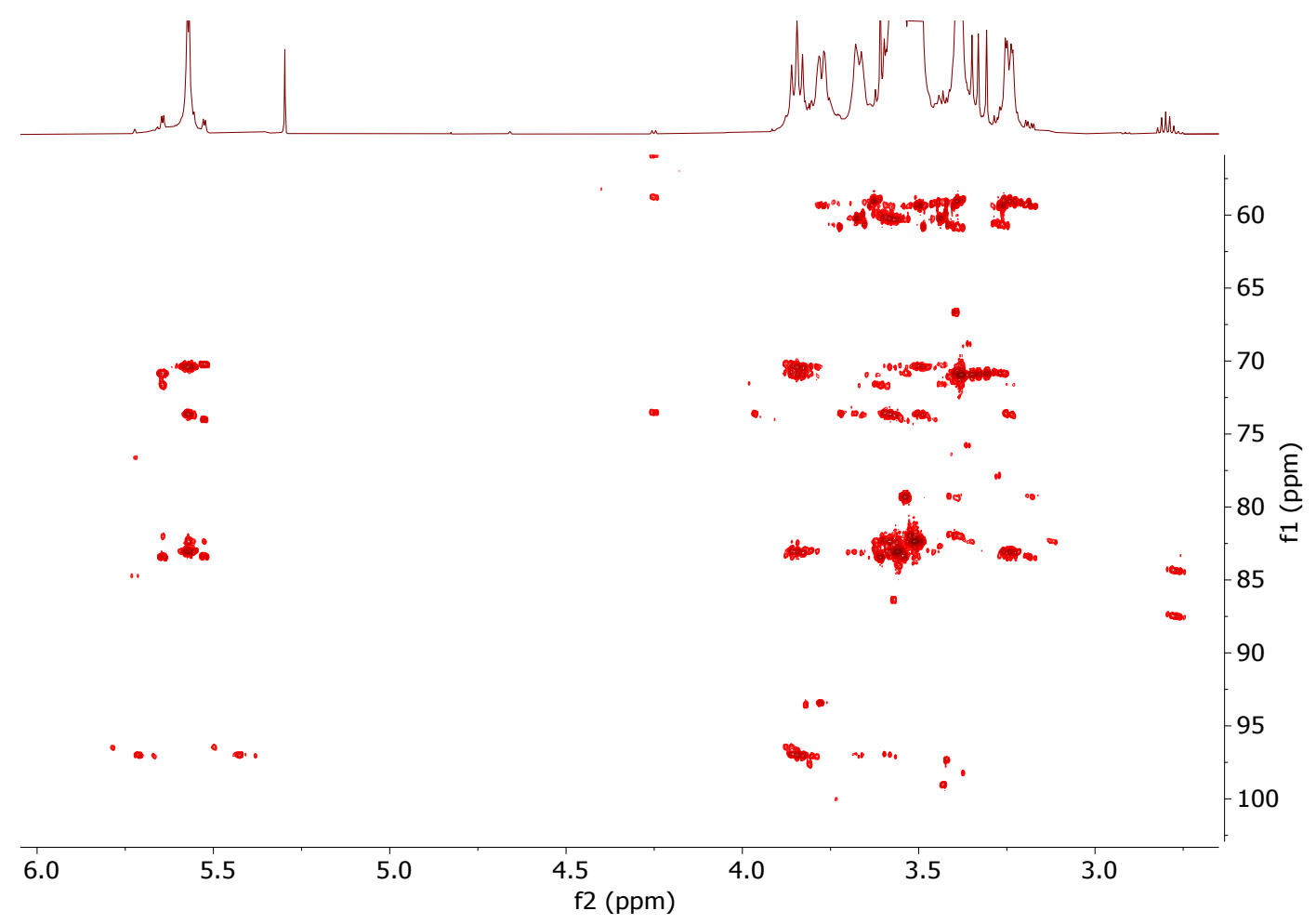

Figure S40: HMBC NMR spectrum of permethylated 1,4- $\alpha$-D-glucan (starch) polysaccharide standard $(5.0 \mathrm{mg})$, acquired in $600 \mu \mathrm{L} \mathrm{CDCl}_{3}$ with $1536 * 256(\mathrm{~F} 2 * \mathrm{~F} 1)$ data points with 48 scans per increment on a Bruker $600 \mathrm{MHz}$ spectrometer equipped with $5 \mathrm{~mm}$ TCI cryoprobe.

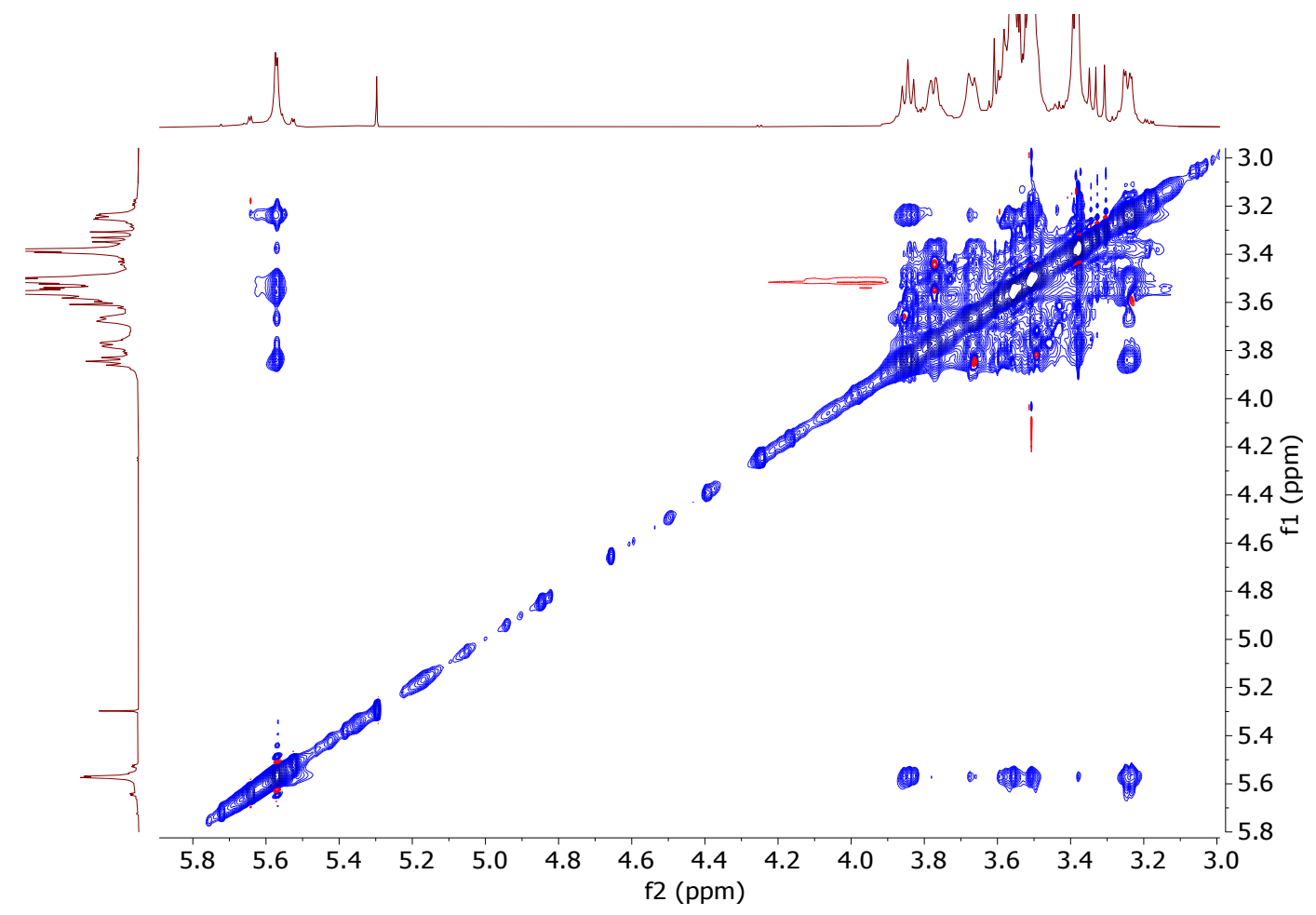

Figure S41: NOESY NMR spectrum of permethylated 1,4- $\alpha$-D-glucan (starch) polysaccharide standard $(5.0 \mathrm{mg})$, acquired in $600 \mu \mathrm{L} \mathrm{CDCl}_{3}$ with $1024 * 256(\mathrm{~F} 2 * \mathrm{~F} 1)$ data points with 48 scans per increment on a Bruker $600 \mathrm{MHz}$ spectrometer equipped with $5 \mathrm{~mm}$ TCI cryoprobe. Mixing time of $80 \mathrm{~ms}$. 


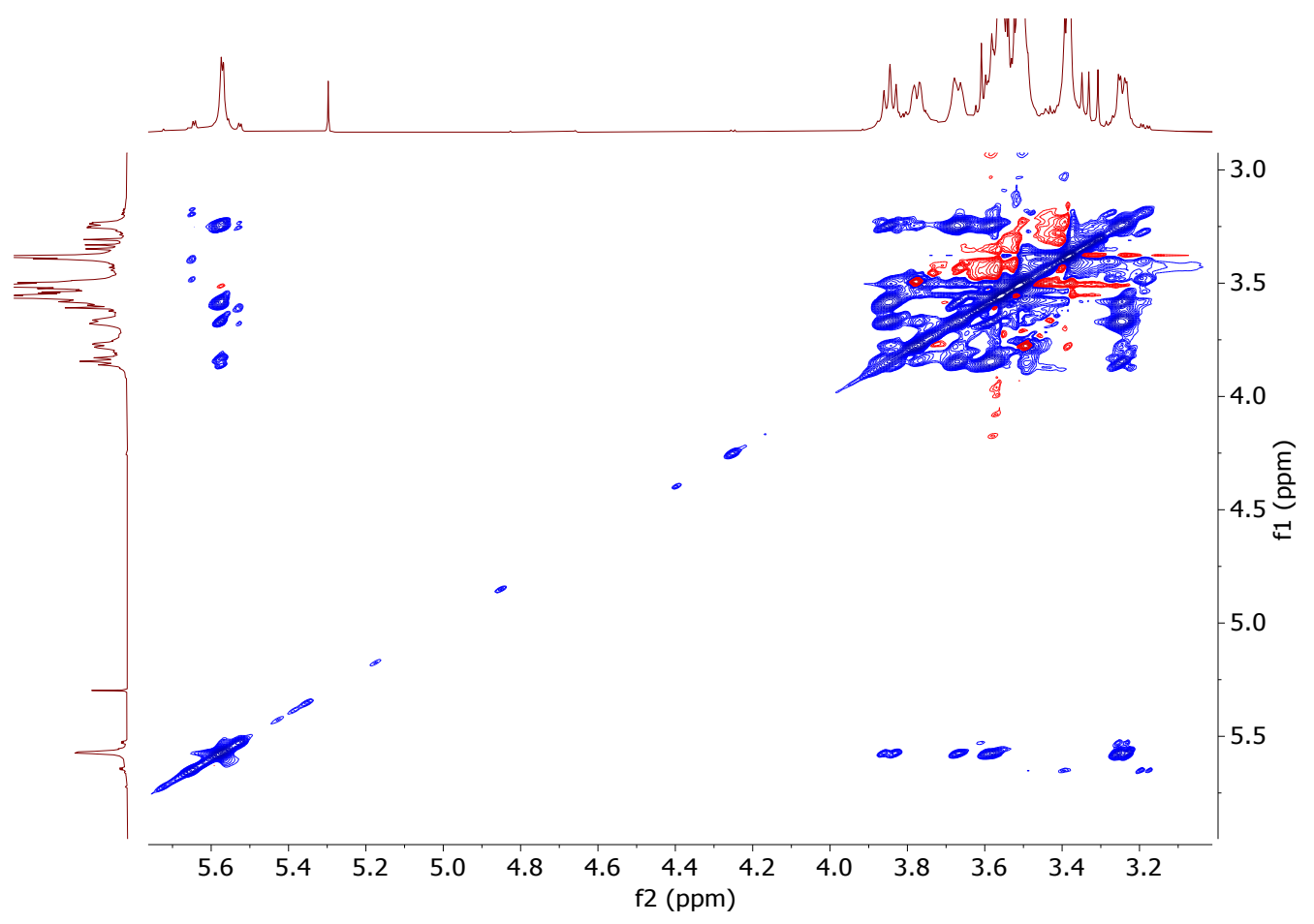

Figure S42: TOCSY NMR spectrum of permethylated 1,4- $\alpha$-D-glucan (starch) polysaccharide standard $(5.0 \mathrm{mg})$, acquired in $600 \mu \mathrm{L} \mathrm{CDCl}_{3}$ with $861 * 384(\mathrm{~F} 2 * \mathrm{~F} 1)$ data points with 28 scans per increment on a Varian $600 \mathrm{MHz}$ spectrometer equipped with $5 \mathrm{~mm}$ VNMRS HNC cryoprobe. Spin-lock time of $80 \mathrm{~ms}$

\section{Dextran NMR Data}

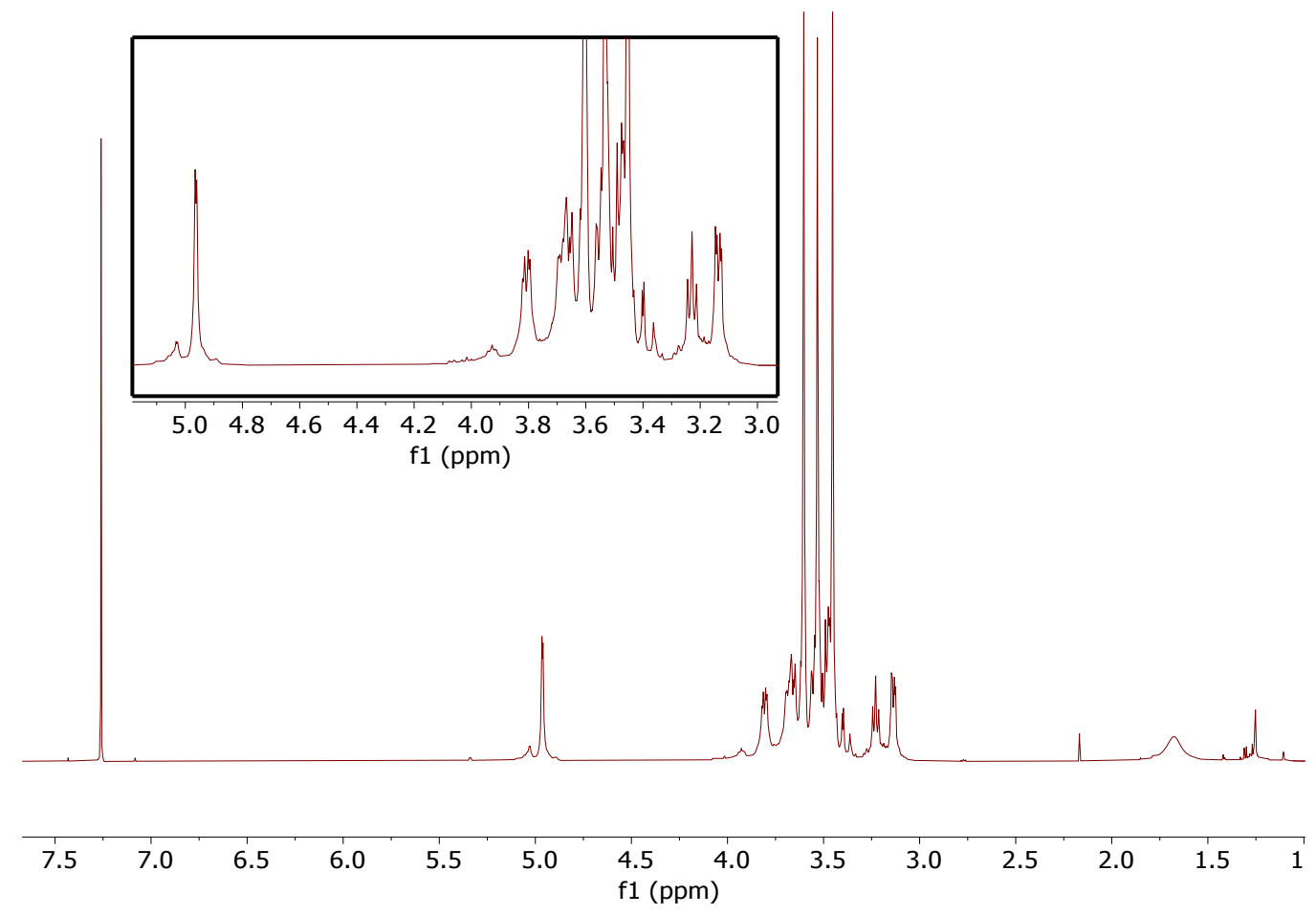

Figure S43: ${ }^{1} \mathrm{H}$ NMR spectrum of permethylated 1,6- $\alpha$-D-glucan (dextran) polysaccharide standard (5.5 $\mathrm{mg}$ ), showing an expanded region of $2.90-5.20 \mathrm{ppm}$, acquired in $600 \mu \mathrm{L} \mathrm{CDCl}_{3}$ with 4 scans on a Bruker $600 \mathrm{MHz}$ spectrometer equipped with $5 \mathrm{~mm}$ TCI cryoprobe. 


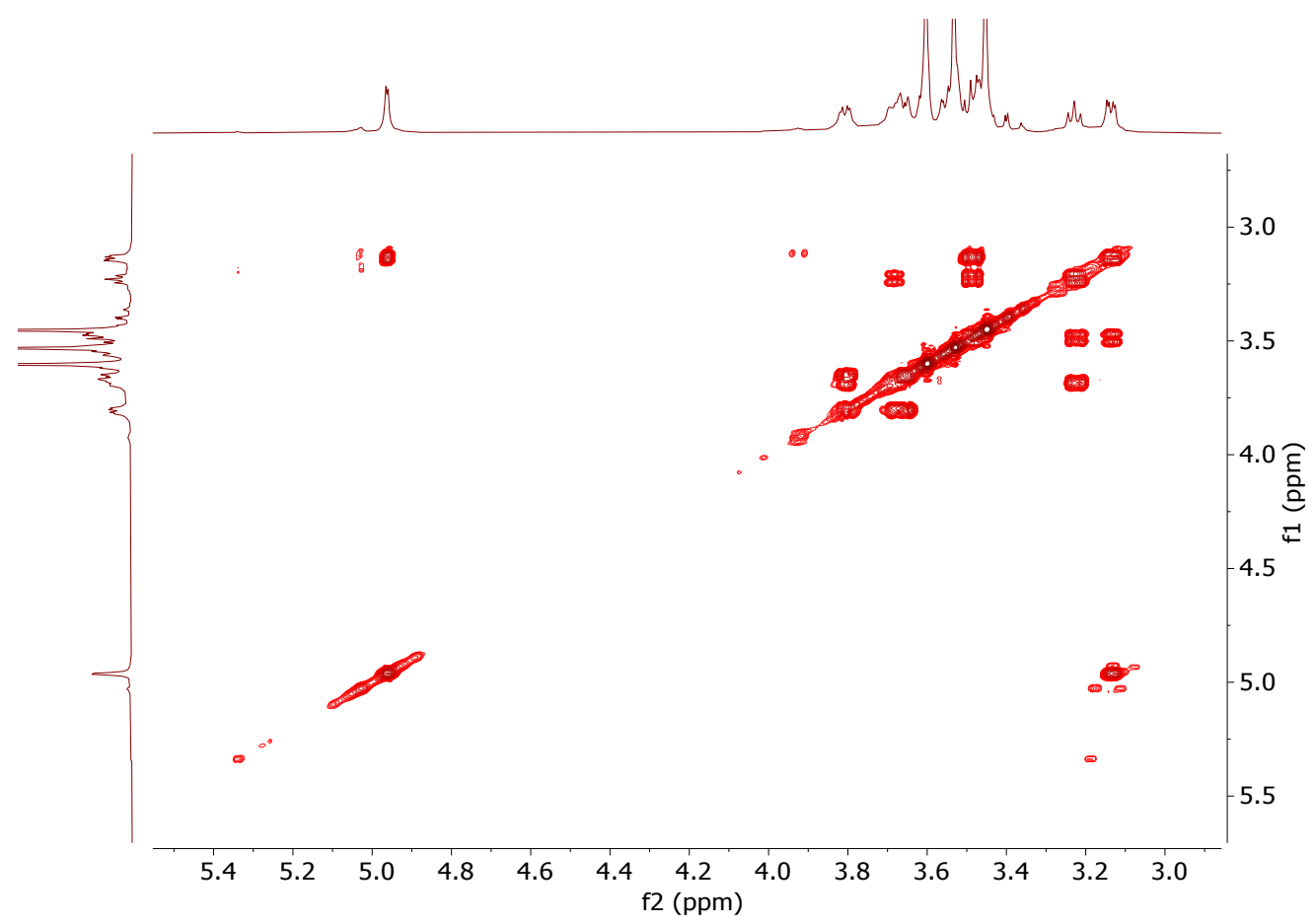

Figure S44: COSY NMR spectrum of permethylated 1,6- $\alpha$-D-glucan (dextran) polysaccharide standard $(5.5 \mathrm{mg})$, acquired in $600 \mu \mathrm{L} \mathrm{CDCl}_{3}$ with $1024 * 512(\mathrm{~F} 2 * \mathrm{~F} 1)$ data points with 2 scans per increment on a Bruker $600 \mathrm{MHz}$ spectrometer equipped with $5 \mathrm{~mm}$ TCI cryoprobe.

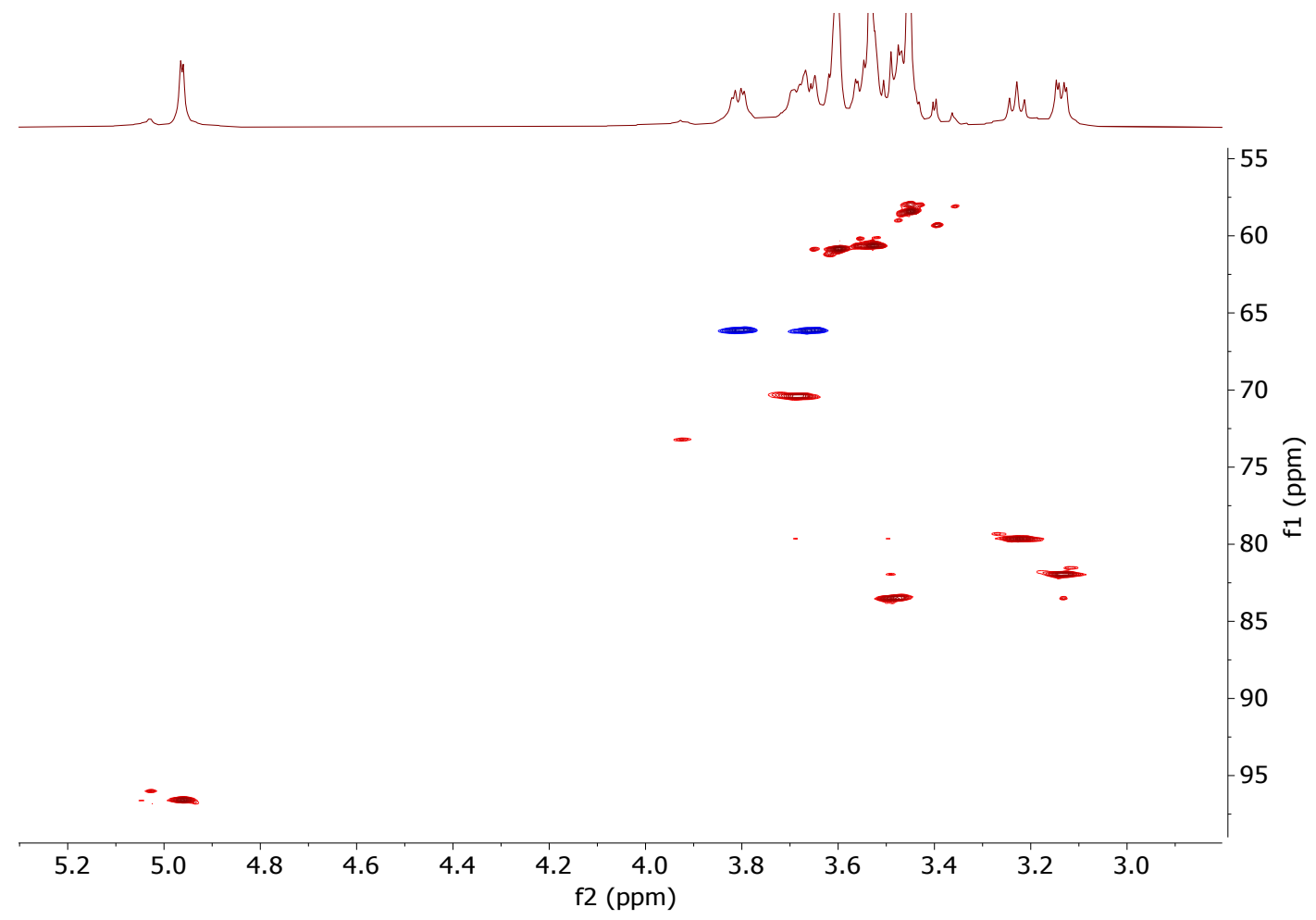

Figure S45: HSQC NMR spectrum of permethylated 1,6- $\alpha$-D-glucan (dextran) polysaccharide standard $(5.5 \mathrm{mg})$, acquired in $600 \mu \mathrm{L} \mathrm{CDCl}_{3}$ with $768 * 768(\mathrm{~F} 2 * \mathrm{~F} 1)$ data points with 2 scans per increment on a Bruker $600 \mathrm{MHz}$ spectrometer equipped with $5 \mathrm{~mm}$ TCI cryoprobe. 


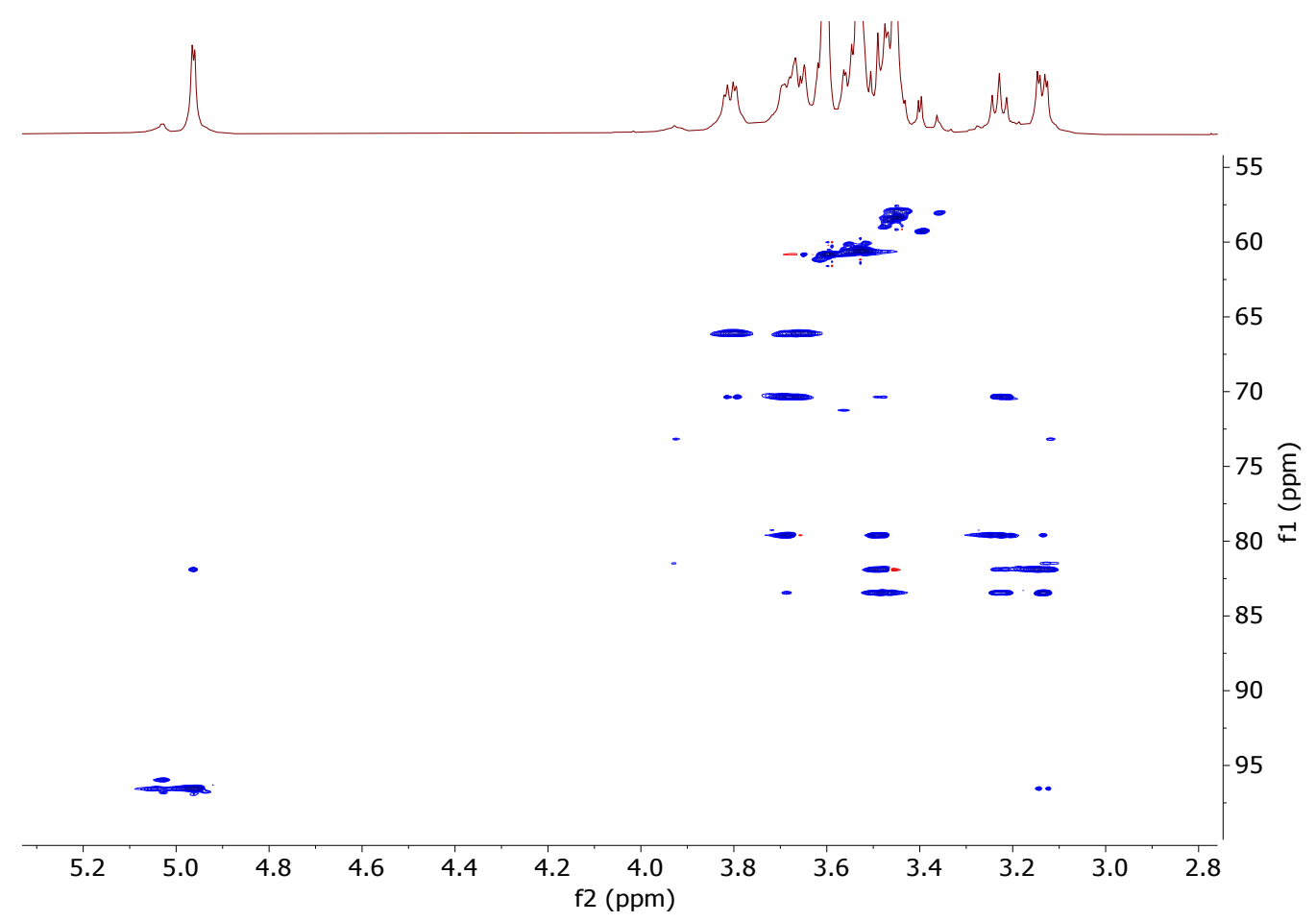

Figure S46: HSQC NMR spectrum of permethylated 1,6- $\alpha$-D-glucan (dextran) polysaccharide standard $(5.5 \mathrm{mg})$, acquired in $600 \mu \mathrm{L} \mathrm{CDCl}_{3}$ with $768 * 768(\mathrm{~F} 2 * \mathrm{~F} 1)$ data points with 8 scans per increment on a Bruker $600 \mathrm{MHz}$ spectrometer equipped with $5 \mathrm{~mm}$ TCI cryoprobe. Mixing time $18 \mathrm{~ms}$.

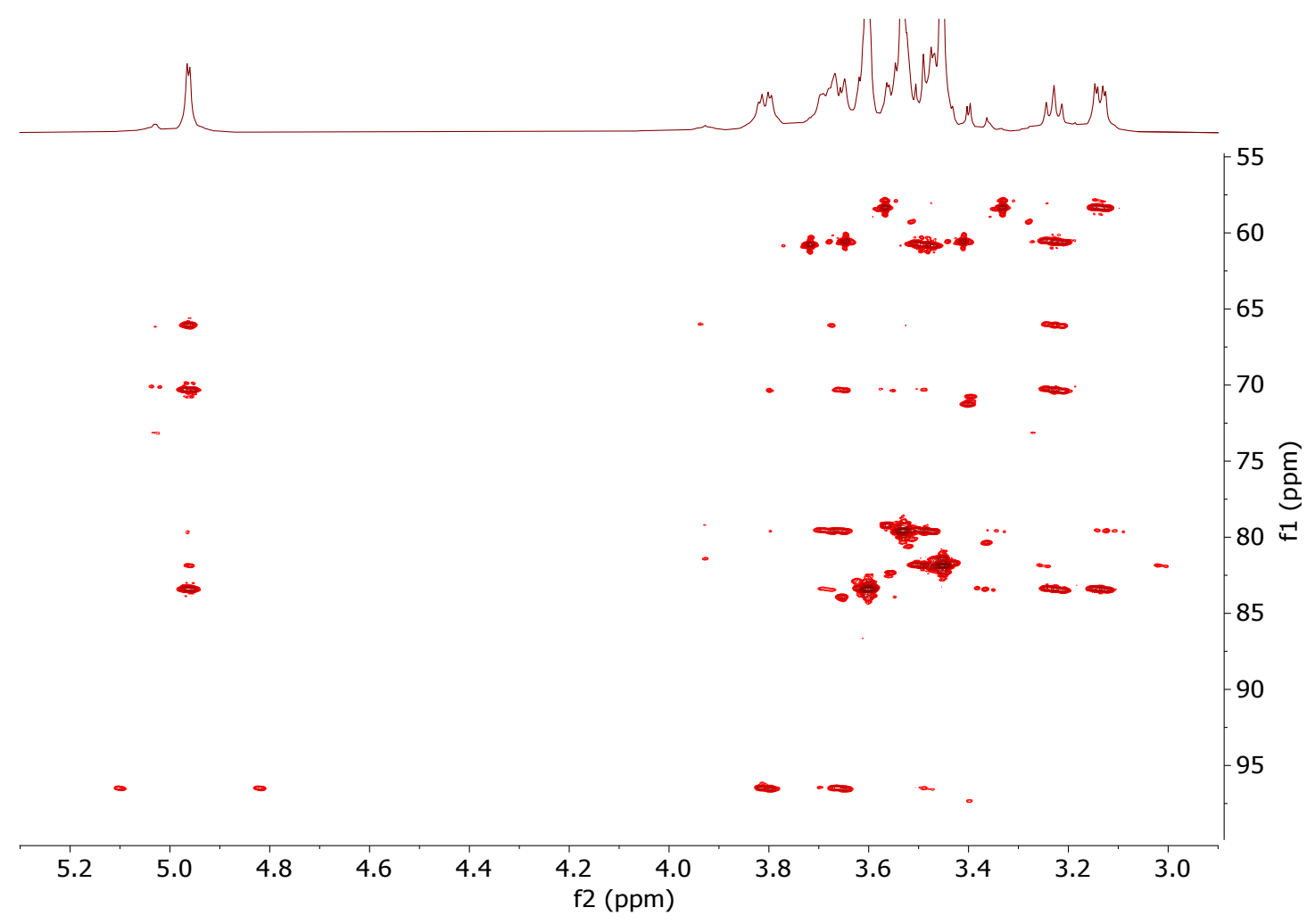

Figure S47: HMBC NMR spectrum of permethylated 1,6- $\alpha$-D-glucan (dextran) polysaccharide standard $(5.5 \mathrm{mg})$, acquired in $600 \mu \mathrm{L} \mathrm{CDCl}_{3}$ with $1536 * 512(\mathrm{~F} 2 * \mathrm{~F} 1)$ data points with 10 scans per increment on a Bruker $600 \mathrm{MHz}$ spectrometer equipped with $5 \mathrm{~mm}$ TCI cryoprobe. 


\section{Galactan NMR Data}

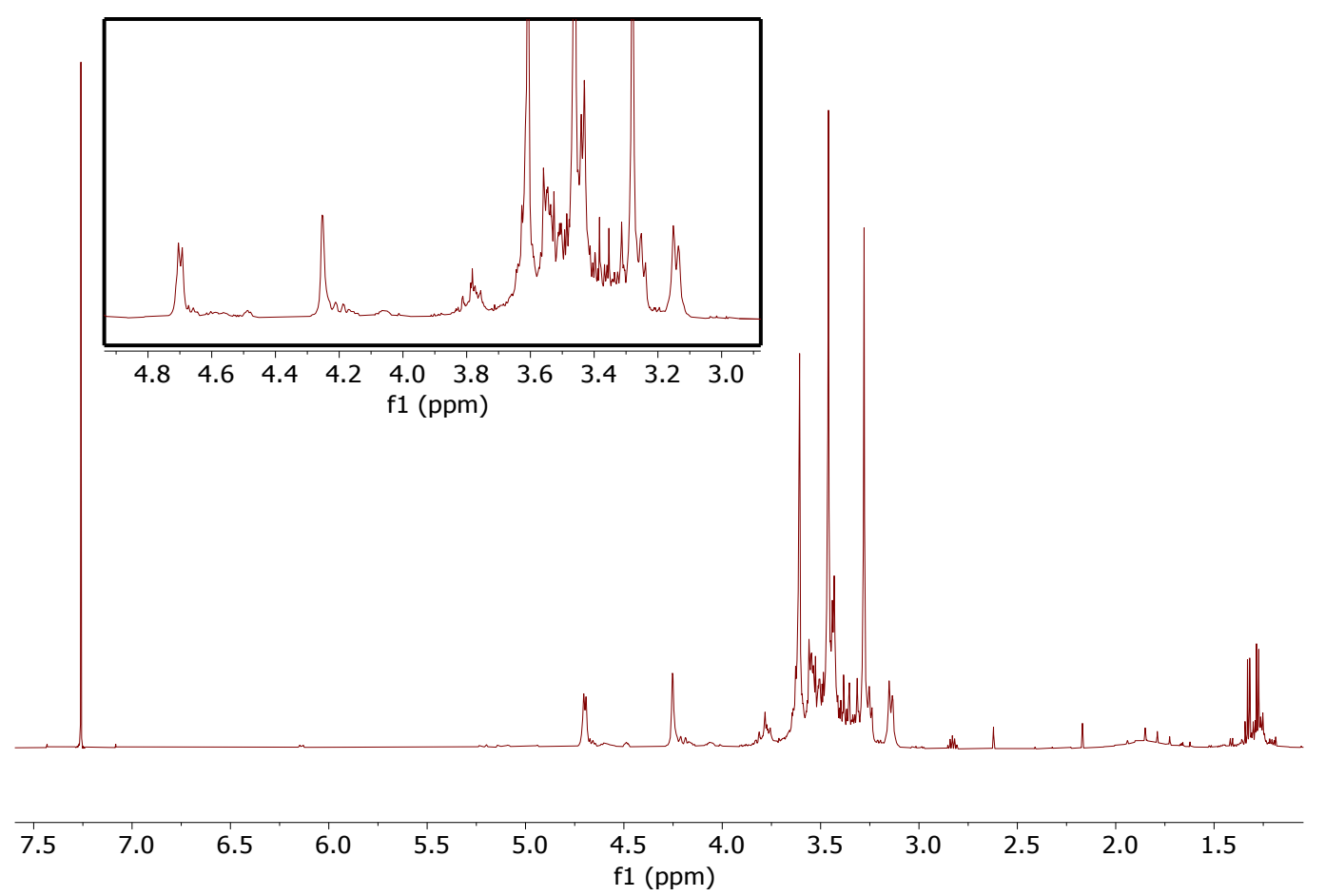

Figure S48: ${ }^{1} \mathrm{H}$ NMR spectrum of permethylated 1,4- $\beta$-D-galactan polysaccharide standard $(8.9 \mathrm{mg})$, showing an expanded region of $2.90-4.90 \mathrm{ppm}$, acquired in $600 \mu \mathrm{L} \mathrm{CDCl}_{3}$ with 2 scans on a VNMRS $600 \mathrm{MHz}$ spectrometer equipped with $5 \mathrm{~mm} \mathrm{HNC}$ cryoprobe.

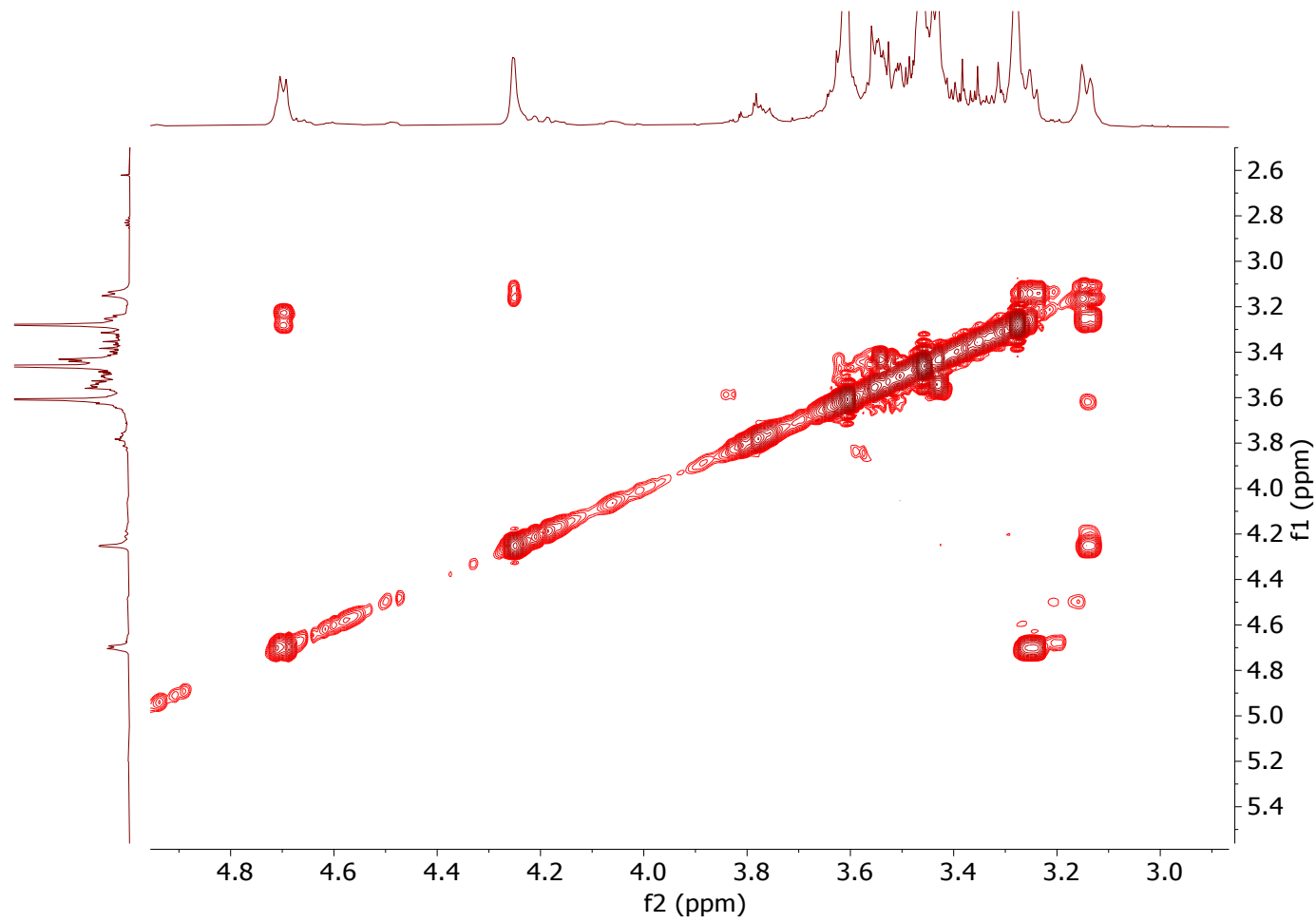

Figure S49: COSY NMR spectrum of permethylated 1,4- $\beta$-D-galactan polysaccharide standard (8.9 $\mathrm{mg}$ ), acquired in $600 \mu \mathrm{L} \mathrm{CDCl}_{3}$ with $962 * 256(\mathrm{~F} 2 * \mathrm{~F} 1)$ data points with 2 scans per increment on a VNMRS $600 \mathrm{MHz}$ spectrometer equipped with $5 \mathrm{~mm}$ HNC cryoprobe. 


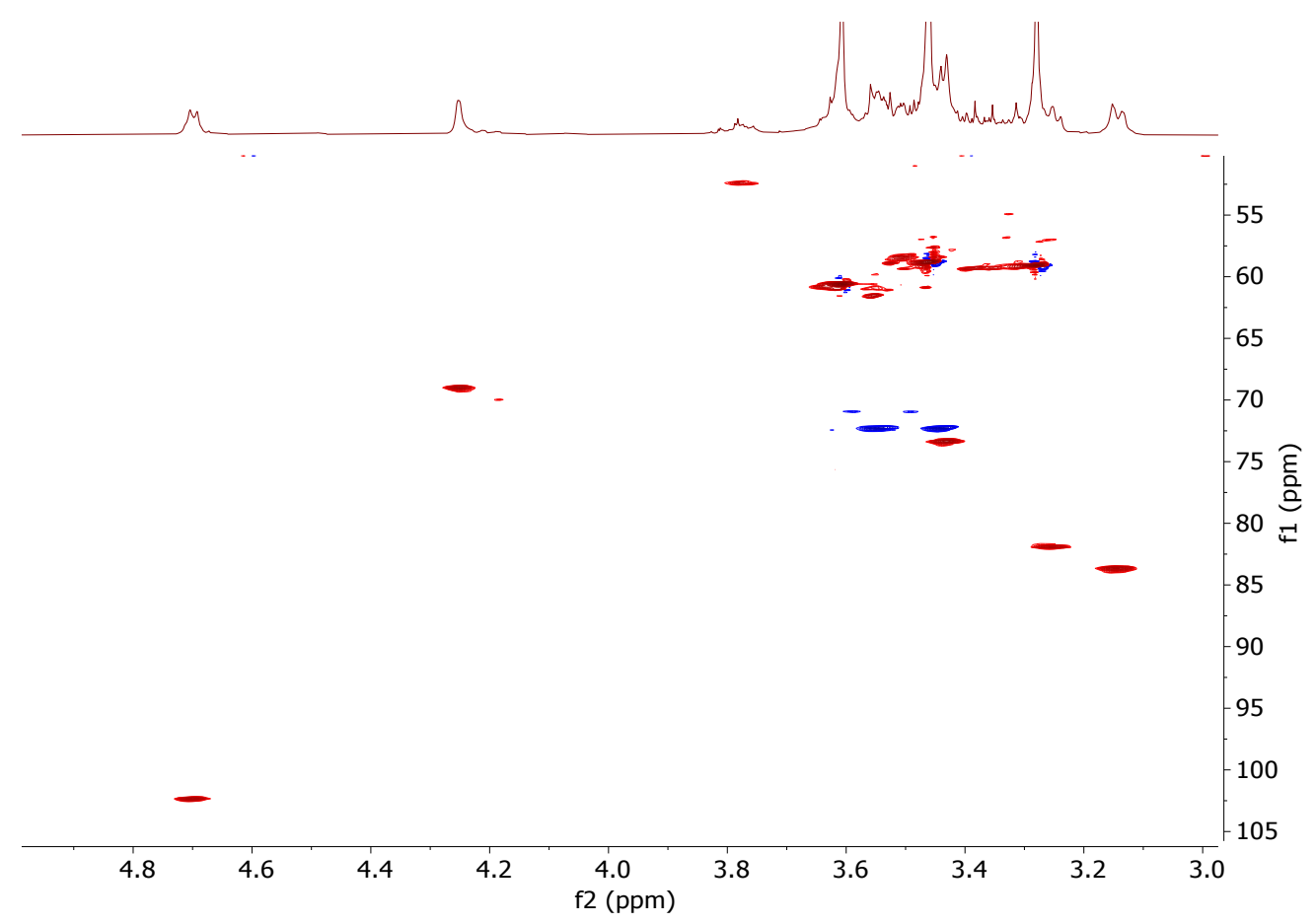

Figure S50: HSQC NMR spectrum of permethylated 1,4- $\beta$-D-galactan polysaccharide standard (8.9 $\mathrm{mg}$ ), acquired in $600 \mu \mathrm{L} \mathrm{CDCl}_{3}$ with $817 * 384(\mathrm{~F} 2 * \mathrm{~F} 1)$ data points with 4 scans per increment on a VNMRS $600 \mathrm{MHz}$ spectrometer equipped with $5 \mathrm{~mm}$ HNC cryoprobe.

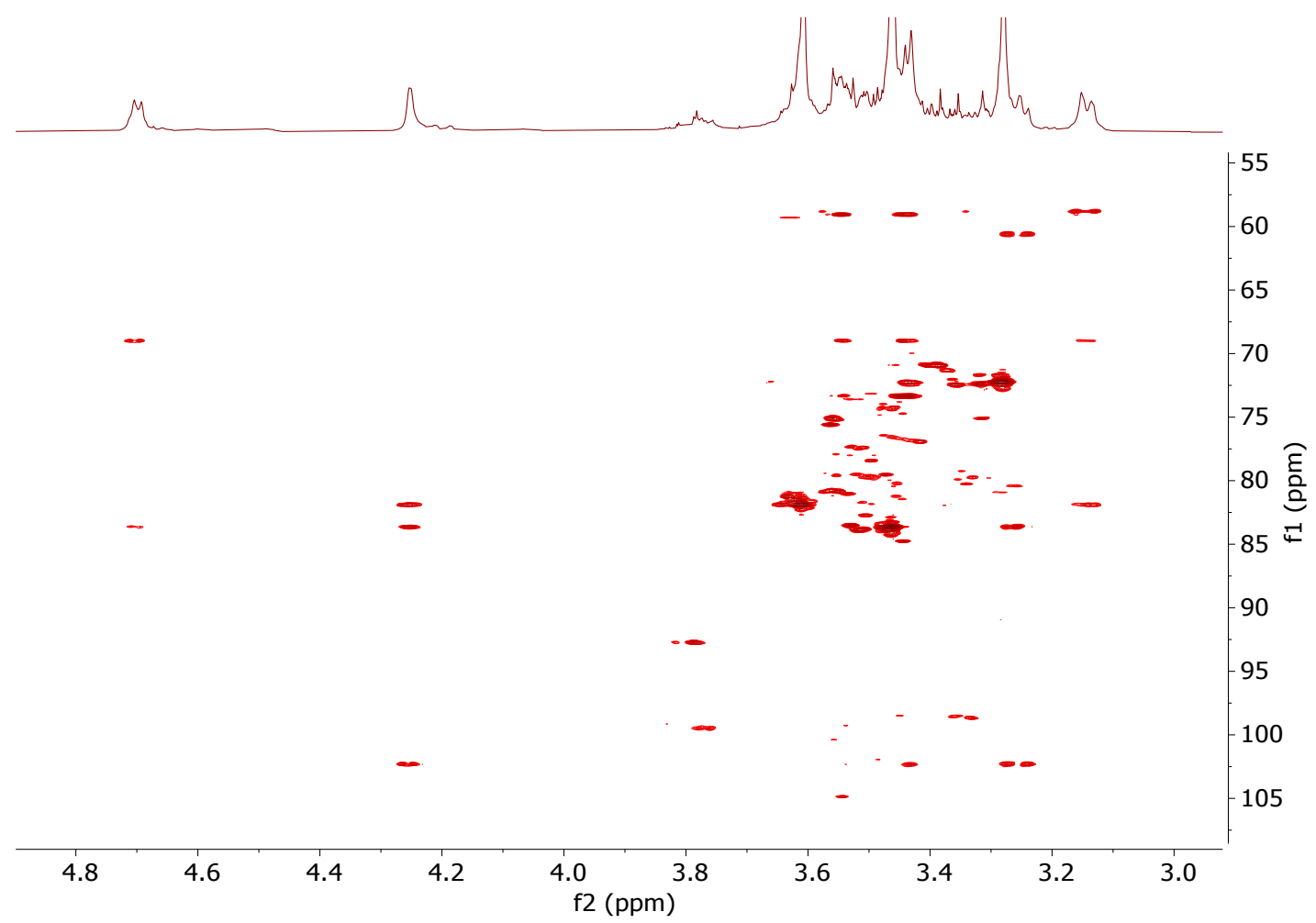

Figure S51: HMBC NMR spectrum of permethylated 1,4- $\beta$-D-galactan polysaccharide standard (8.9 $\mathrm{mg}$ ), acquired in $600 \mu \mathrm{L} \mathrm{CDCl}_{3}$ with $1202 * 384(\mathrm{~F} 2 * \mathrm{~F} 1)$ data points with 8 scans per increment on a VNMRS $600 \mathrm{MHz}$ spectrometer equipped with $5 \mathrm{~mm}$ HNC cryoprobe. 


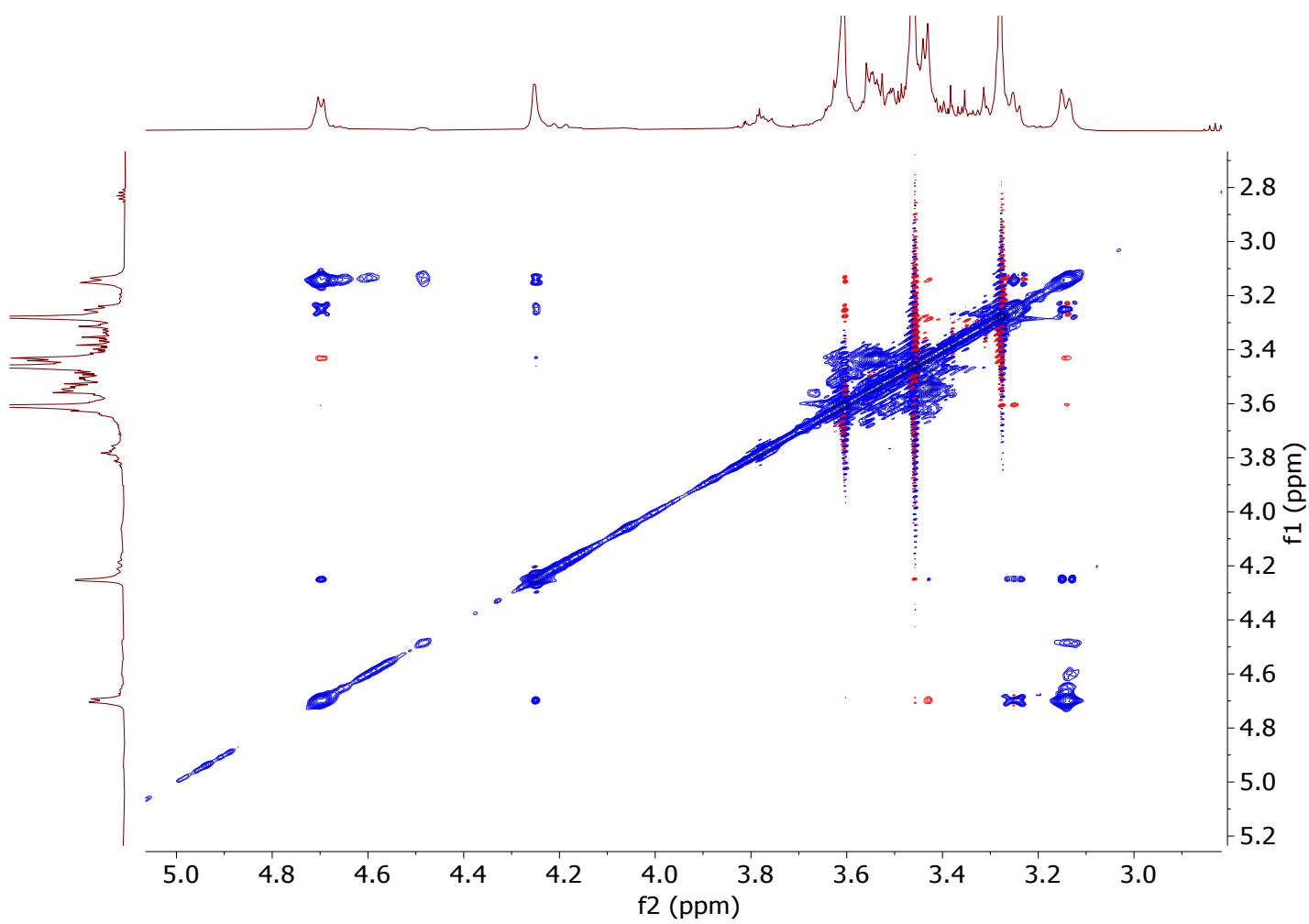

Figure S52: TOCSY NMR spectrum of permethylated 1,4- $\beta$-D-galactan polysaccharide standard (8.9 $\mathrm{mg}$ ), acquired in $600 \mu \mathrm{L} \mathrm{CDCl}{ }_{3}$ with $1202 * 384(\mathrm{~F} 2 * \mathrm{~F} 1)$ data points with 4 scans per increment on a VNMRS $600 \mathrm{MHz}$ spectrometer equipped with $5 \mathrm{~mm}$ HNC cryoprobe. Spin-lock time $80 \mathrm{~ms}$.

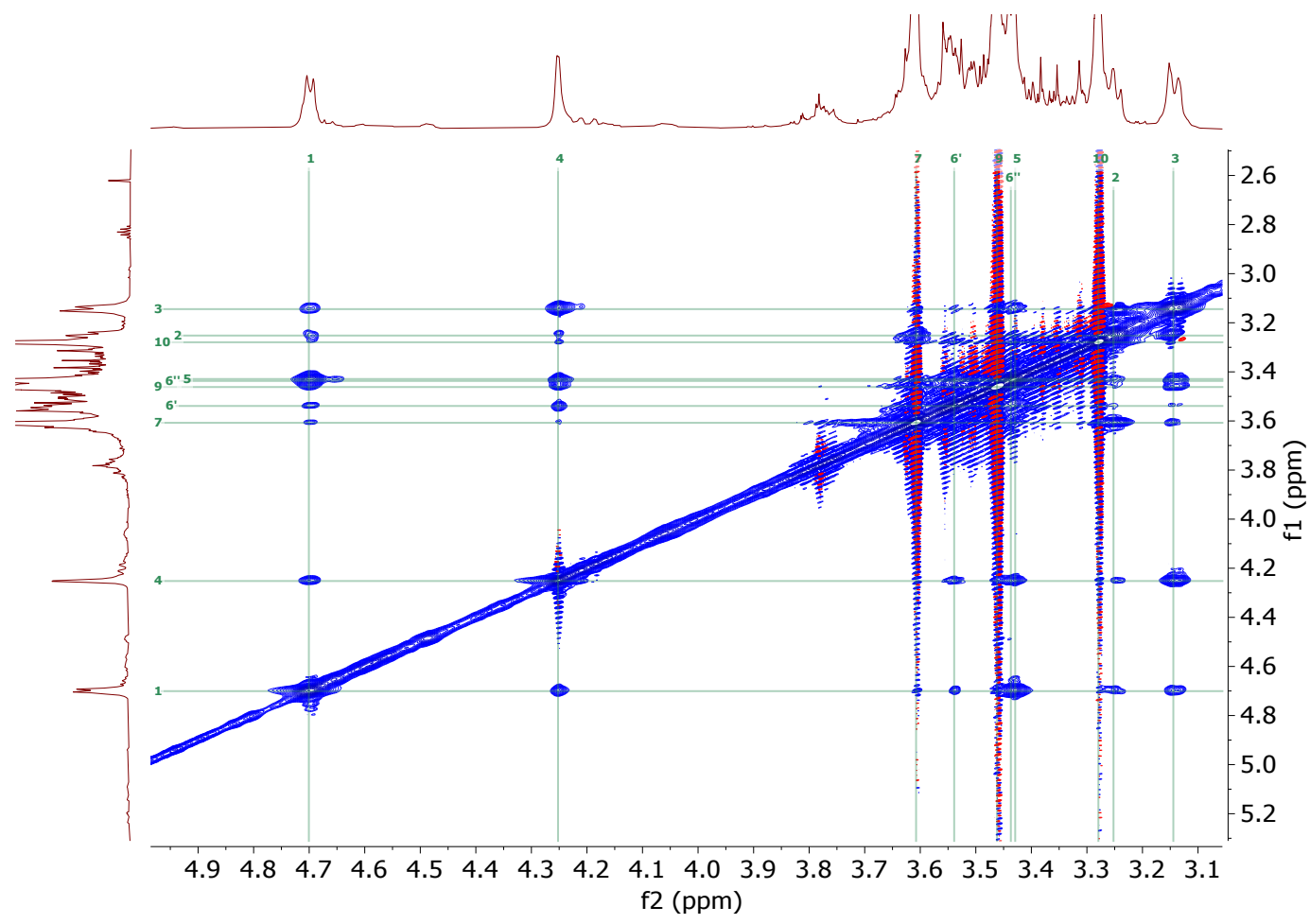

Figure S53: NOESY NMR spectrum of permethylated 1,4- $\beta$-D-galactan polysaccharide standard (8.9 $\mathrm{mg}$ ), acquired in $600 \mu \mathrm{L} \mathrm{CDCl}{ }_{3}$ with $1202 * 384(\mathrm{~F} 2 * \mathrm{~F} 1)$ data points with 8 scans per increment on a VNMRS $600 \mathrm{MHz}$ spectrometer equipped with $5 \mathrm{~mm}$ HNC cryoprobe. Mixing time $80 \mathrm{~ms}$. 


\section{Mixed Glucan NMR Data}
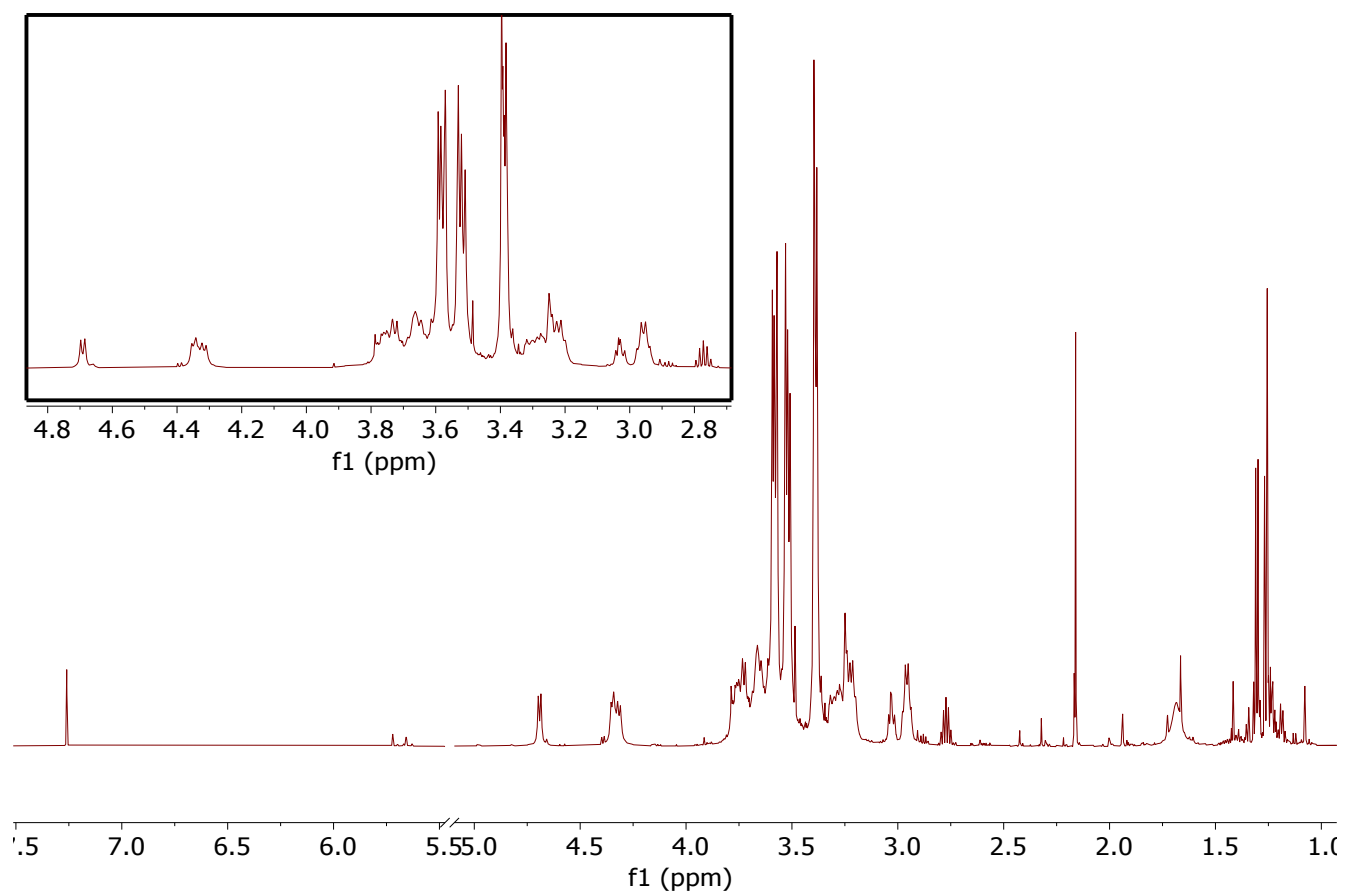

Figure S54: ${ }^{1} \mathrm{H}$ NMR spectrum of permethylated mixed-glucan (1,3- and 1,4- $\beta$-D-glucan) polysaccharide standard, showing an expanded region of $2.70-4.80 \mathrm{ppm}$, acquired in $600 \mu \mathrm{L} \mathrm{CDCl} \mathrm{Cl}_{3}$ with 4 scans on a Bruker $600 \mathrm{MHz}$ spectrometer equipped with $5 \mathrm{~mm}$ TCI cryoprobe. Large DCM signal at $5.30 \mathrm{ppm}$ truncated.

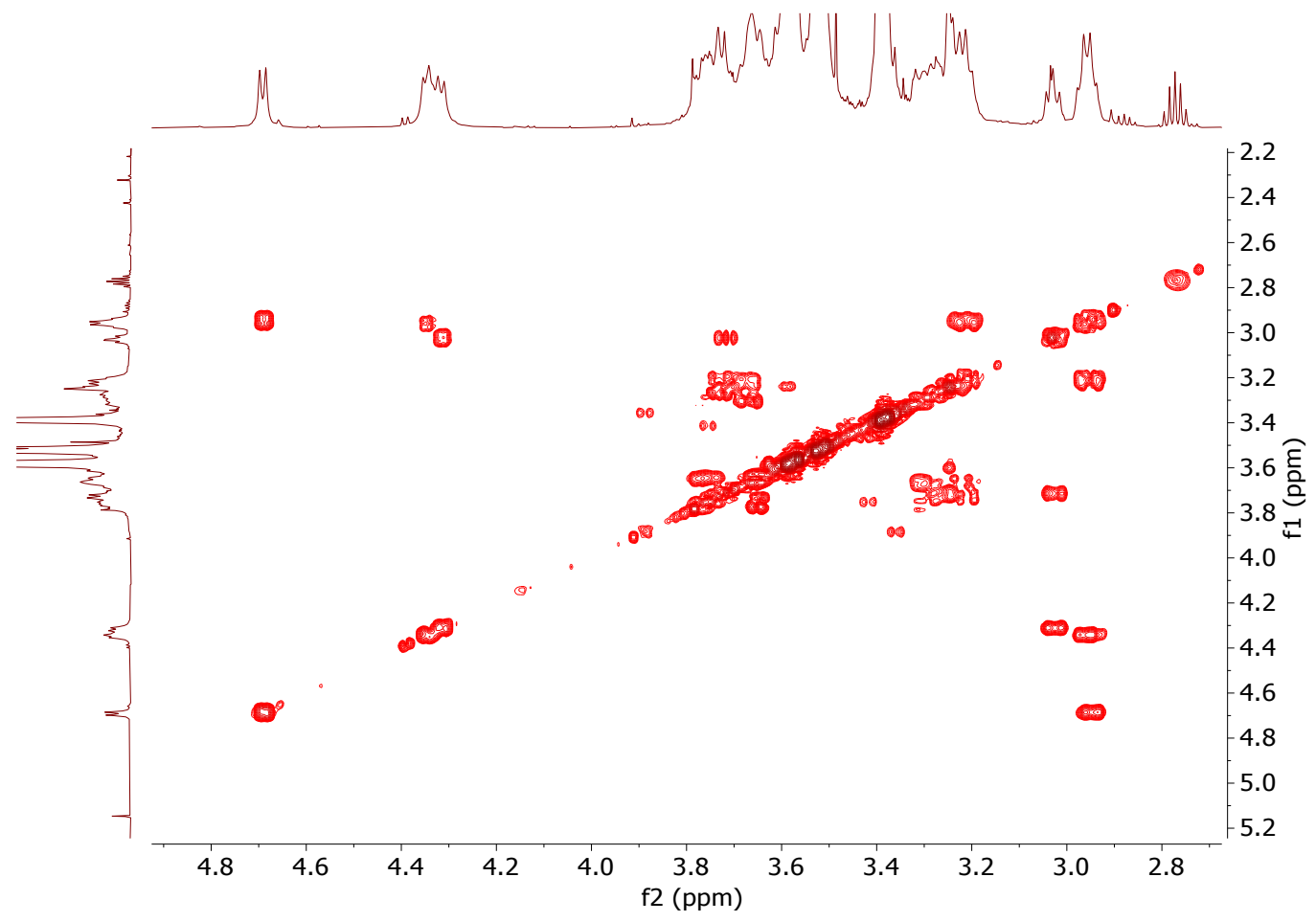

Figure S55: COSY NMR spectrum of permethylated mixed-glucan (1,3- and 1,4- $\beta$-D-glucan) polysaccharide standard acquired in $600 \mu \mathrm{L} \mathrm{CDCl}_{3}$ with $1024 * 384(\mathrm{~F} 2 * \mathrm{~F} 1)$ data points with 4 scans per increment on a Bruker $600 \mathrm{MHz}$ spectrometer equipped with $5 \mathrm{~mm}$ TCI cryoprobe. 


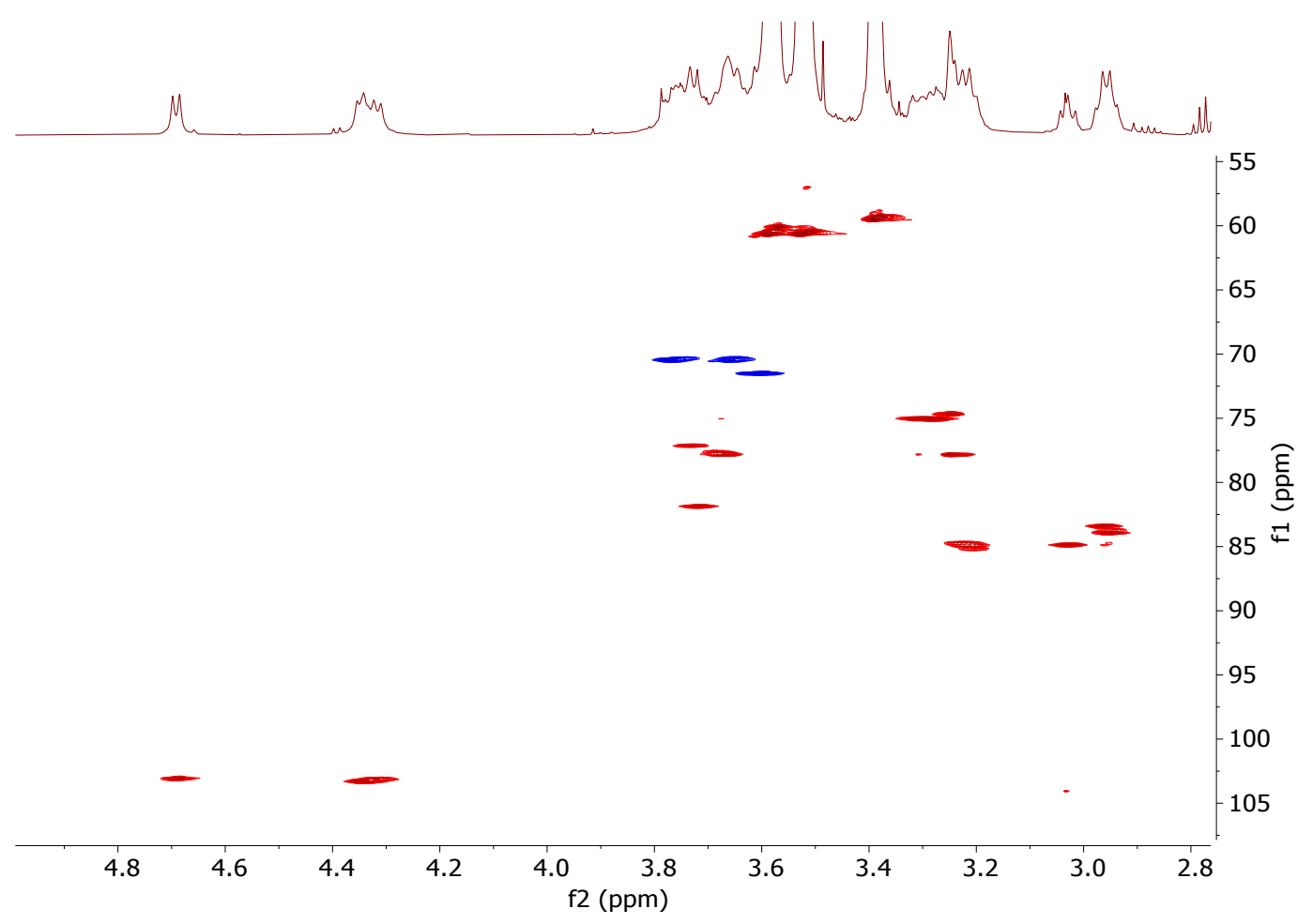

Figure S56: HSQC NMR spectrum of permethylated mixed-glucan (1,3- and 1,4- $\beta$-D-glucan) polysaccharide standard acquired in $600 \mu \mathrm{L} \mathrm{CDCl}_{3}$ with $768 * 768(\mathrm{~F} 2 * \mathrm{~F} 1)$ data points with 4 scans per increment on a Bruker $600 \mathrm{MHz}$ spectrometer equipped with $5 \mathrm{~mm}$ TCI cryoprobe.

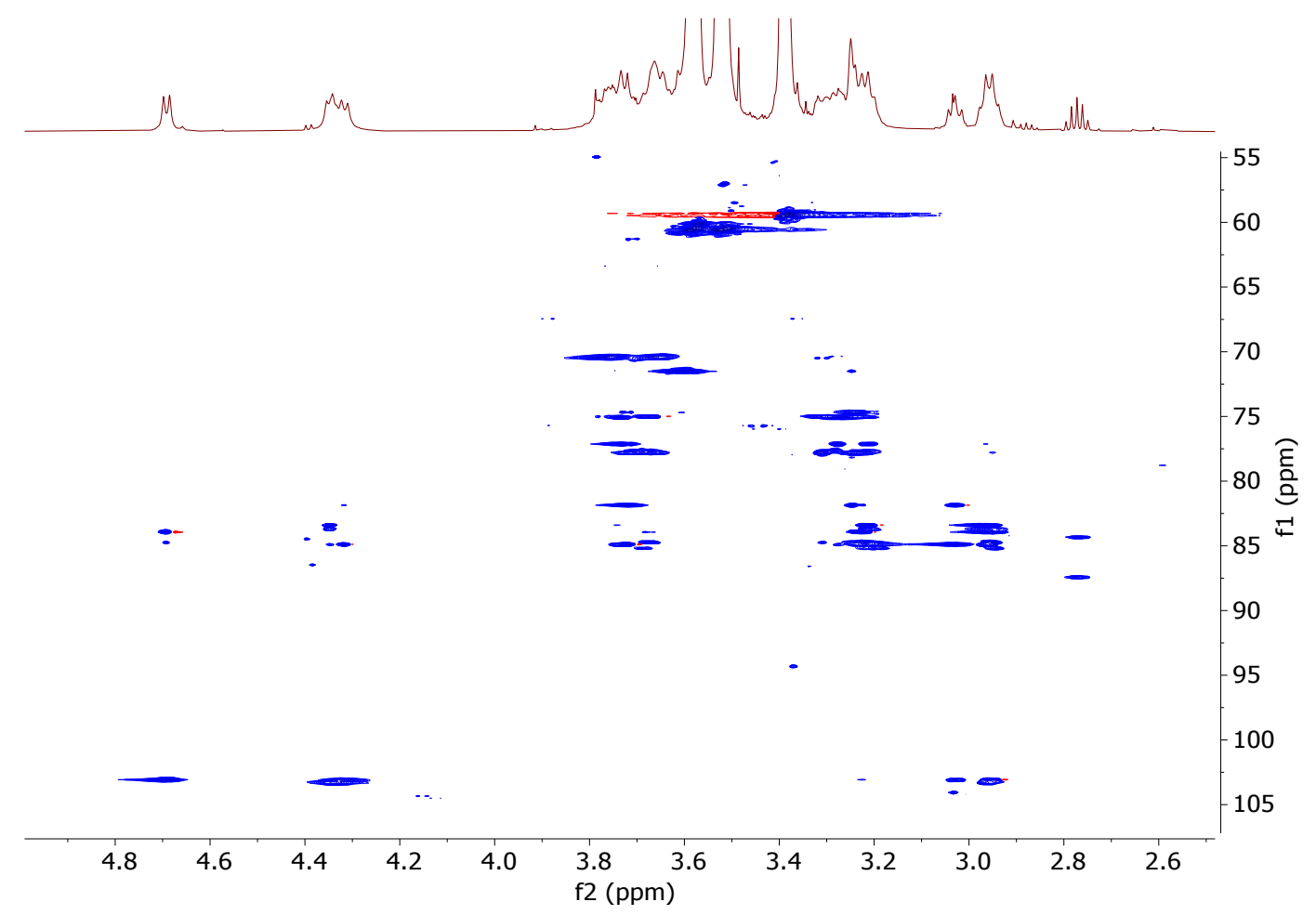

Figure S57: HSQC-TOCSY NMR spectrum of permethylated mixed-glucan (1,3- and 1,4- $\beta$-D-glucan) polysaccharide standard acquired in $600 \mu \mathrm{L} \mathrm{CDCl}_{3}$ with $768 * 768(\mathrm{~F} 2 * \mathrm{~F} 1)$ data points with 8 scans per increment on a Bruker $600 \mathrm{MHz}$ spectrometer equipped with $5 \mathrm{~mm}$ TCI cryoprobe. Mixing time 18 ms. 


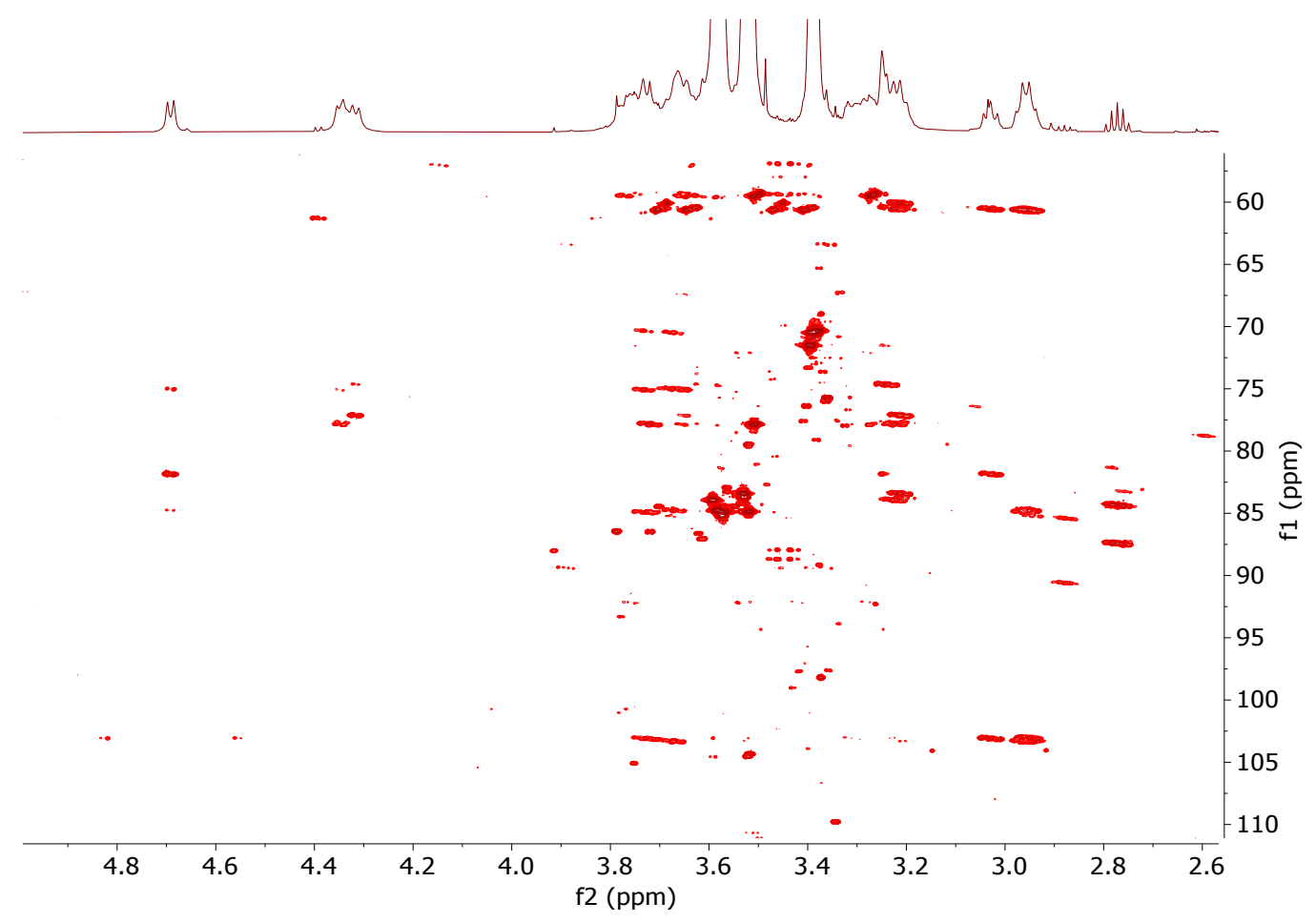

Figure S58: HMBC NMR spectrum of permethylated mixed-glucan (1,3- and 1,4- $\beta$-D-glucan) polysaccharide standard acquired in $600 \mu \mathrm{L} \mathrm{CDCl}_{3}$ with $1536 * 512(\mathrm{~F} 2 * \mathrm{~F} 1)$ data points with 20 scans per increment on a Bruker $600 \mathrm{MHz}$ spectrometer equipped with $5 \mathrm{~mm}$ TCI cryoprobe.

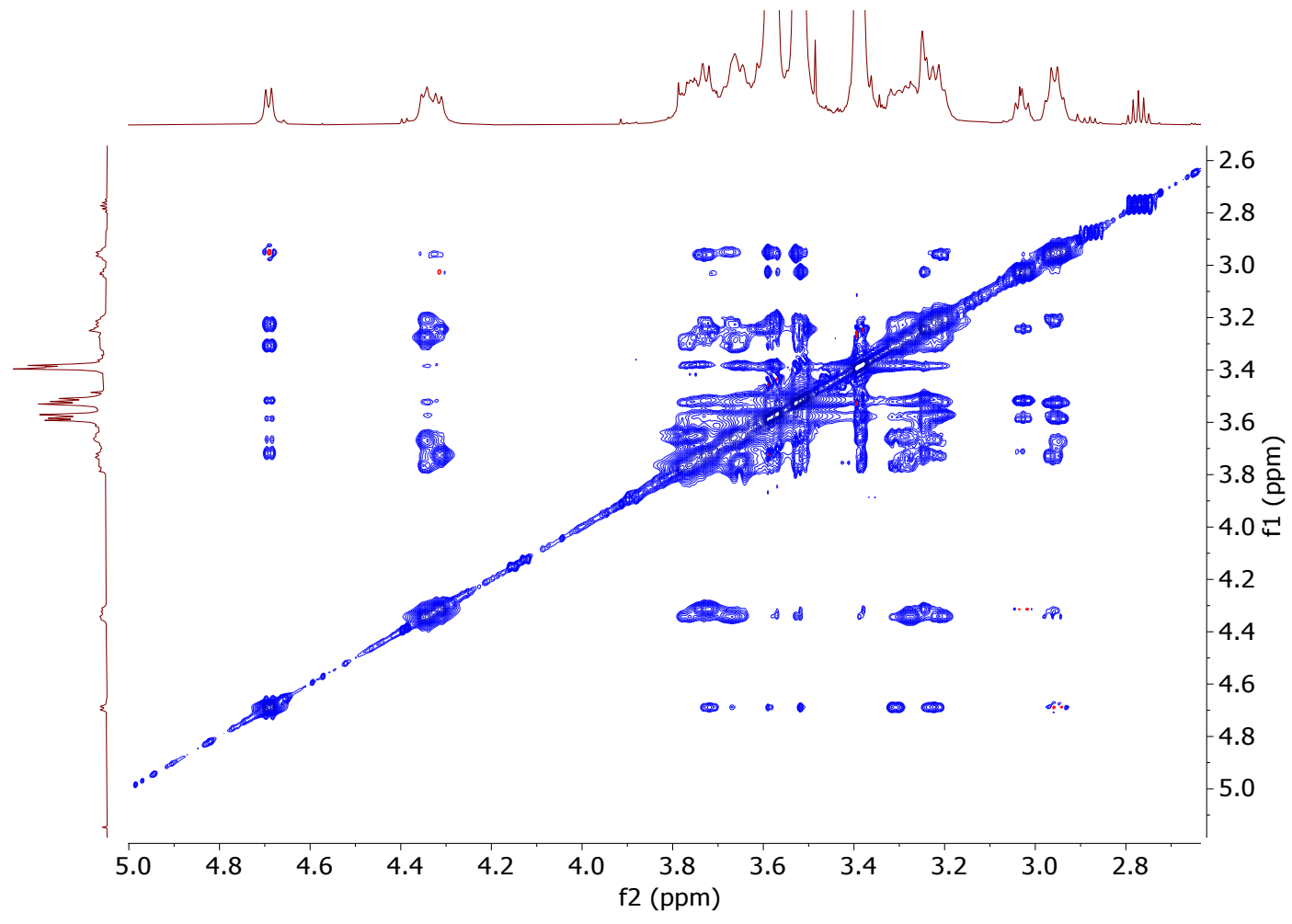

Figure S59: NOESY NMR spectrum of permethylated mixed-glucan (1,3- and 1,4- $\beta$-D-glucan) polysaccharide standard acquired in $600 \mu \mathrm{L} \mathrm{CDCl}_{3}$ with $1536 * 768(\mathrm{~F} 2 * \mathrm{~F} 1)$ data points with 16 scans per increment on a Bruker $600 \mathrm{MHz}$ spectrometer equipped with $5 \mathrm{~mm}$ TCI cryoprobe. Mixing time 80 ms. 


\section{Arabinoxylan NMR Data}

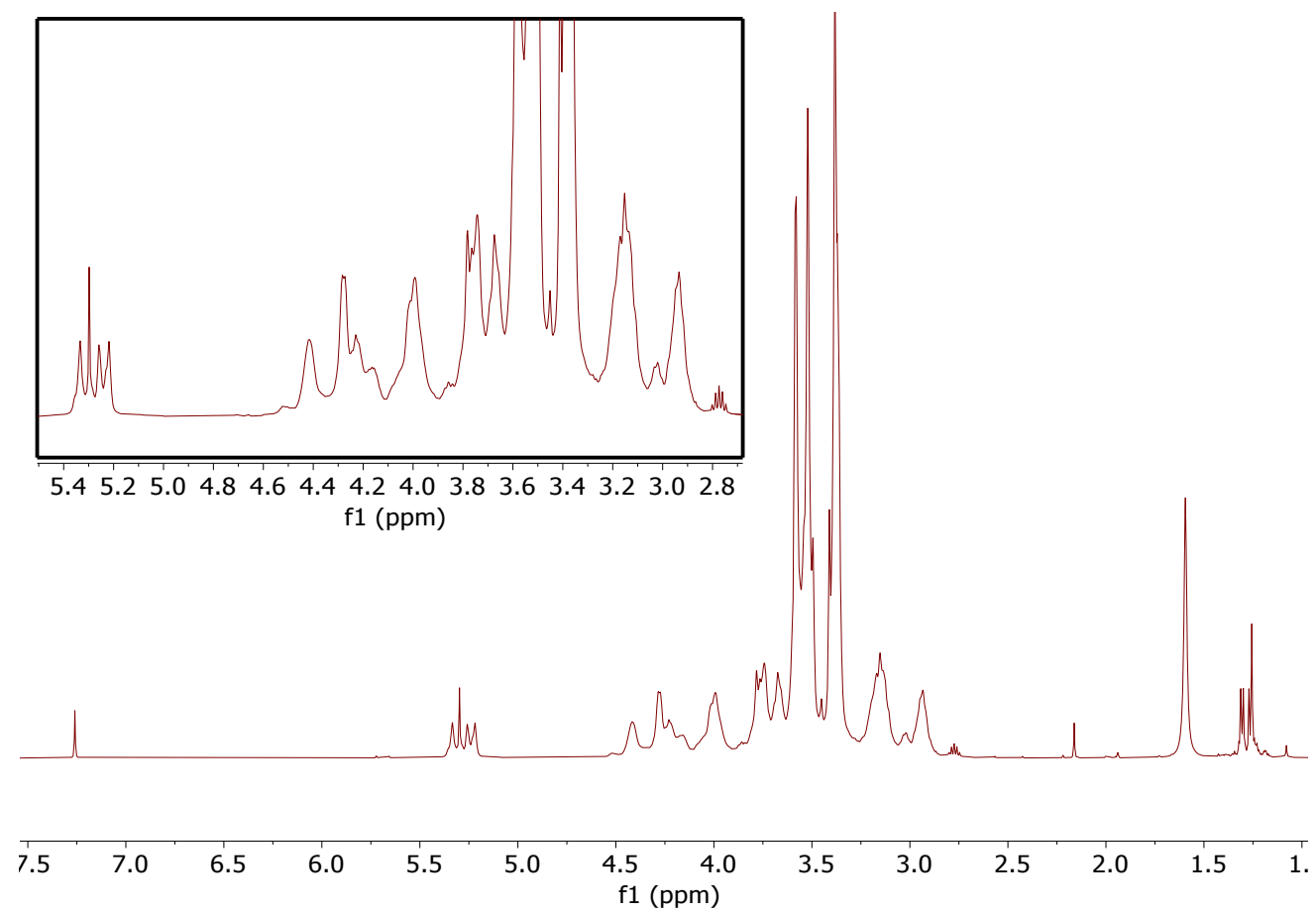

Figure S60: ${ }^{1} \mathrm{H}$ NMR spectrum of permethylated arabinoxylan polysaccharide standard $(8.7 \mathrm{mg})$, showing an expanded region of $2.70-5.50 \mathrm{ppm}$, acquired in $600 \mu \mathrm{L} \mathrm{CDCl}_{3}$ with 16 scans on a Inova $500 \mathrm{MHz}$ spectrometer equipped with $5 \mathrm{~mm} \mathrm{HNC}$ room temperature probe.

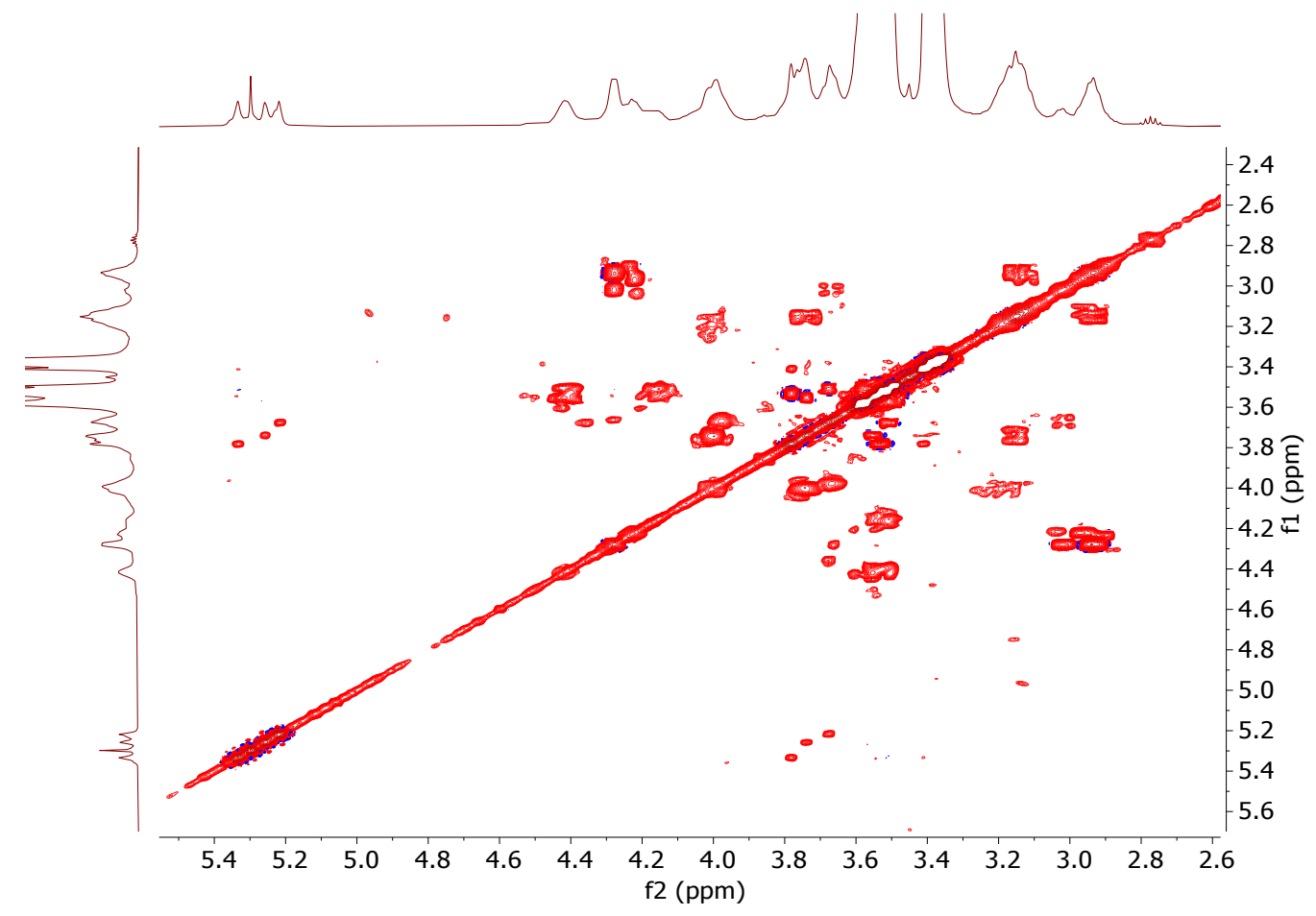

Figure S61: COSY NMR spectrum of permethylated arabinoxylan polysaccharide standard $(8.7 \mathrm{mg})$, acquired in $600 \mu \mathrm{L} \mathrm{CDCl}_{3}$ with $1065 * 512(\mathrm{~F} 2 * \mathrm{~F} 1)$ data points with 4 scans per increment on an Inova $500 \mathrm{MHz}$ spectrometer equipped with $5 \mathrm{~mm} \mathrm{HNC}$ room temperature probe. 


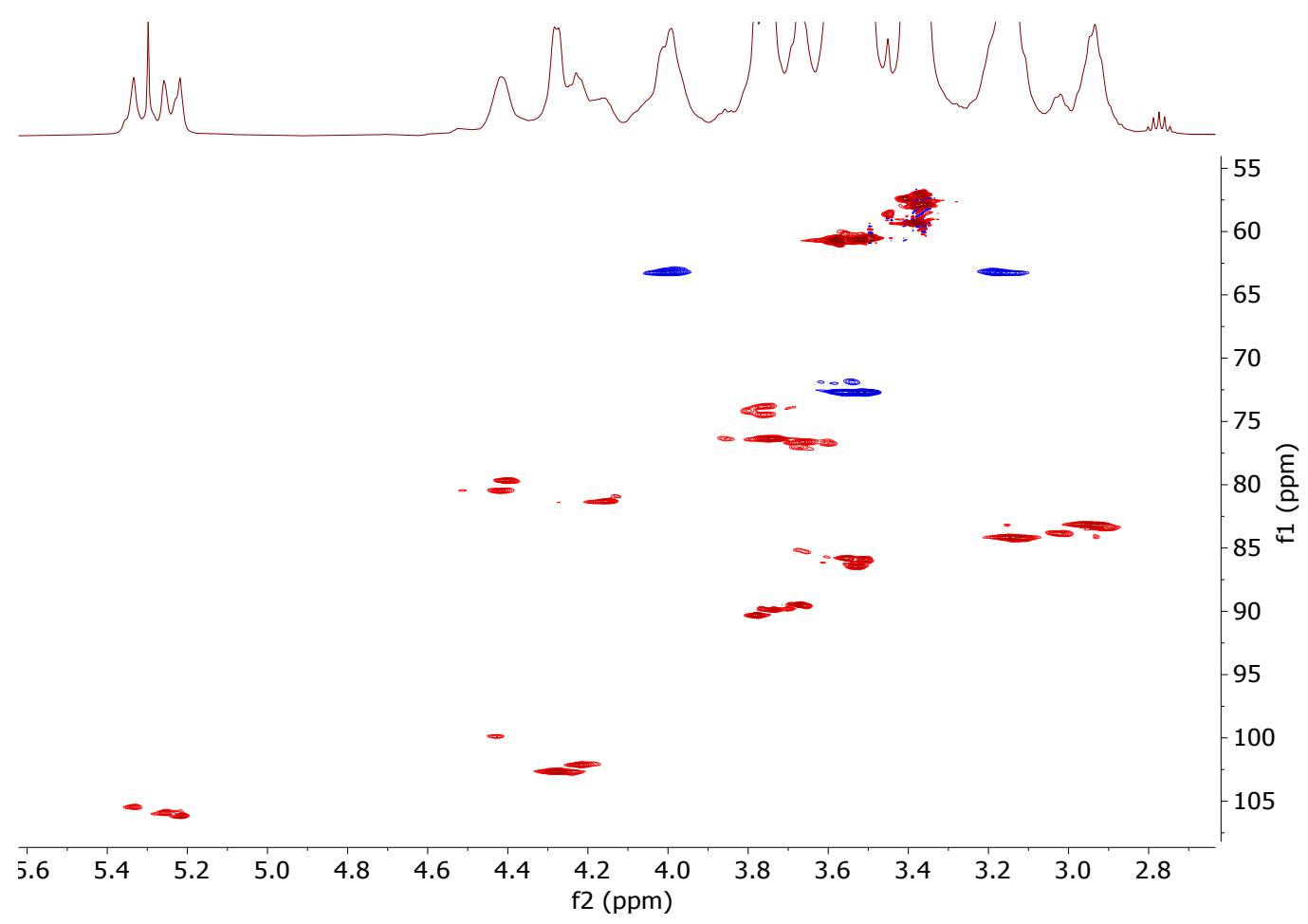

Figure S62: HSQC NMR spectrum of permethylated arabinoxylan polysaccharide standard $(8.7 \mathrm{mg})$, acquired in $600 \mu \mathrm{L} \mathrm{CDCl}_{3}$ with $768 * 256(\mathrm{~F} 2 * \mathrm{~F} 1)$ data points with 32 scans per increment on a Bruker $600 \mathrm{MHz}$ spectrometer equipped with $5 \mathrm{~mm}$ TCI cryoprobe.

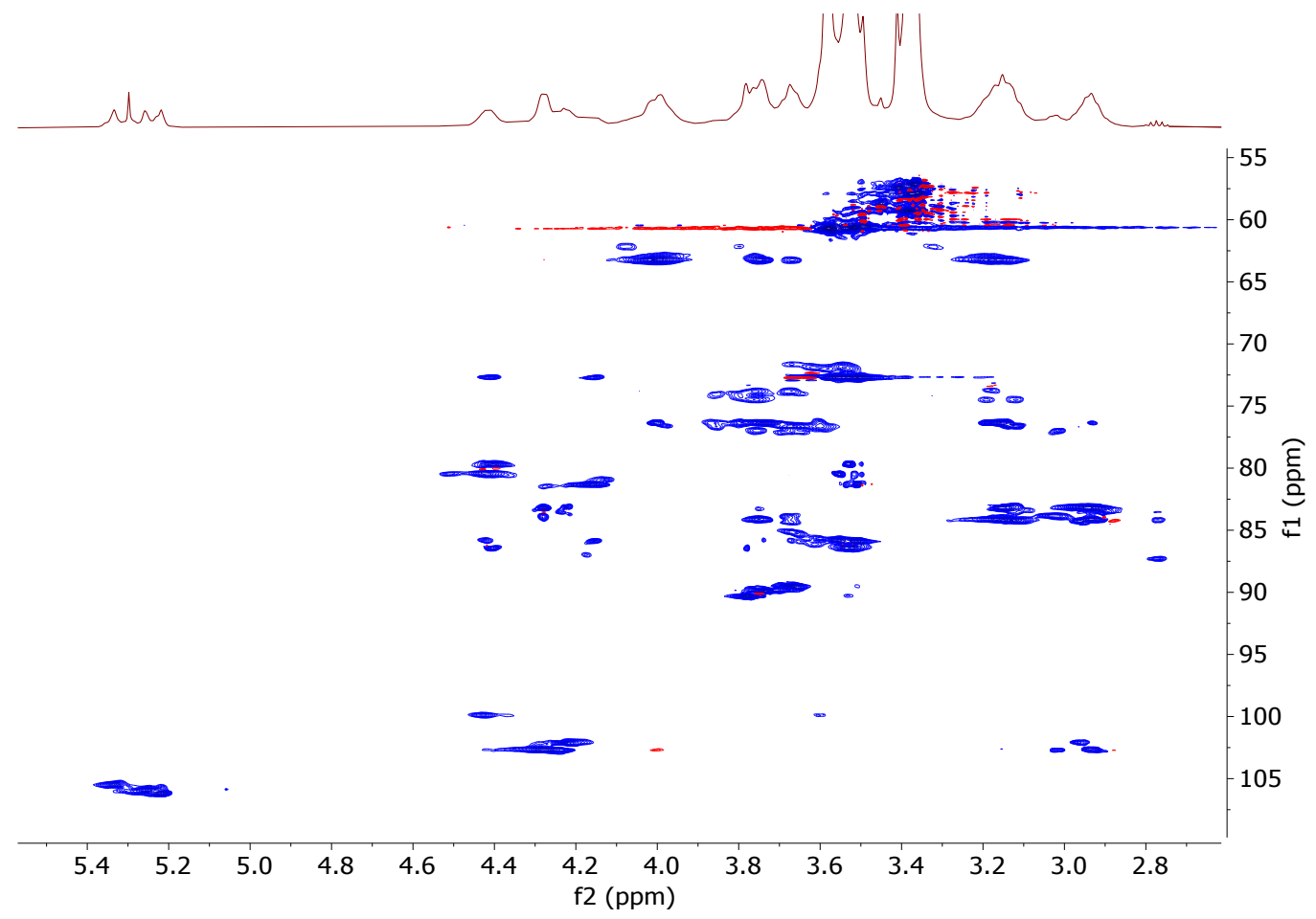

Figure S63: HSQC-TOCSY NMR spectrum of permethylated arabinoxylan polysaccharide standard $(8.7 \mathrm{mg})$, acquired in $600 \mu \mathrm{L} \mathrm{CDCl}_{3}$ with $768 * 256(\mathrm{~F} 2 * \mathrm{~F} 1)$ data points with 48 scans per increment on a Bruker $600 \mathrm{MHz}$ spectrometer equipped with $5 \mathrm{~mm}$ TCI cryoprobe. Mixing time is $18 \mathrm{~ms}$. 


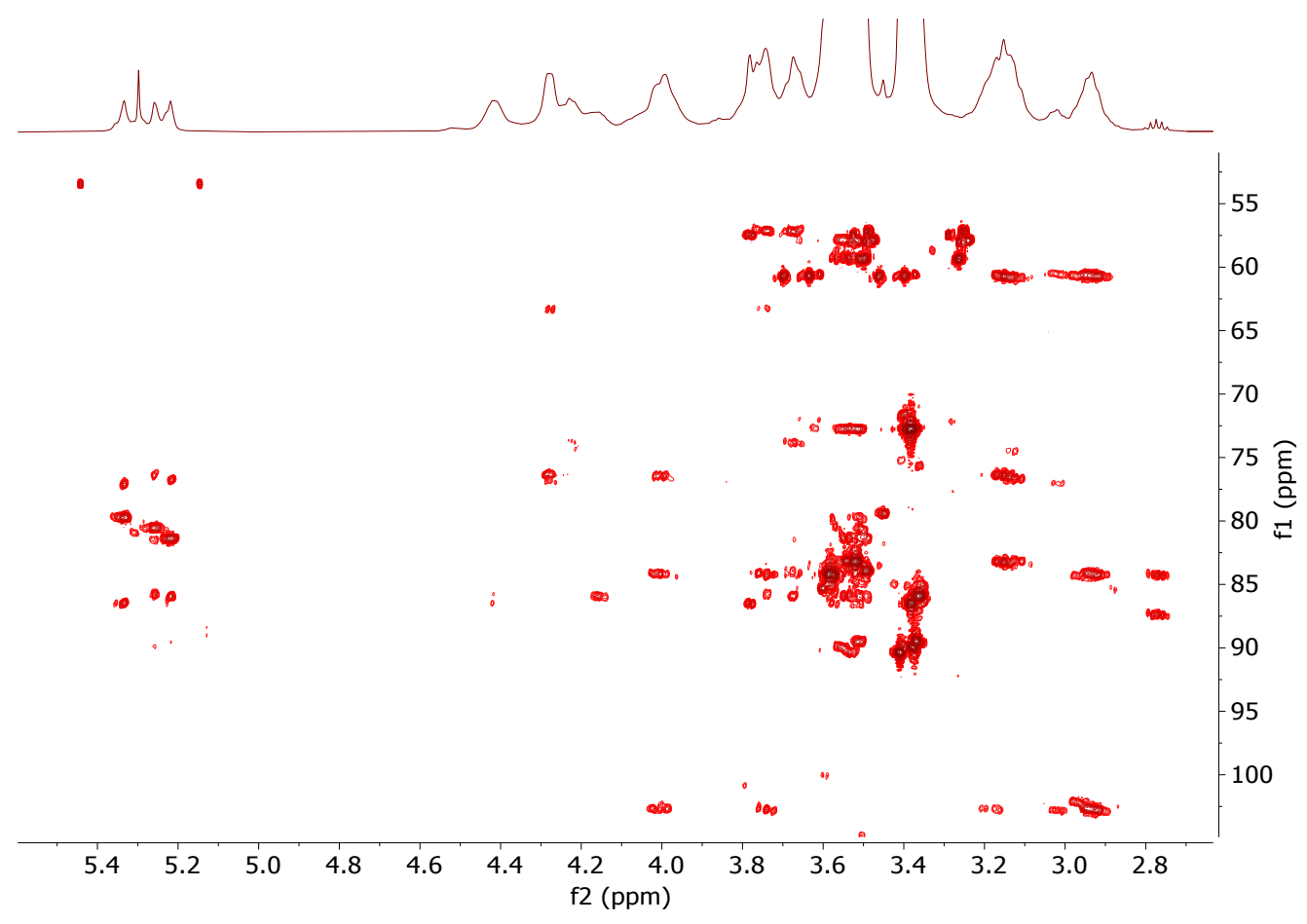

Figure S64: HMBC NMR spectrum of permethylated arabinoxylan polysaccharide standard $(8.7 \mathrm{mg})$, acquired in $600 \mu \mathrm{L} \mathrm{CDCl}_{3}$ with $1024 * 166(\mathrm{~F} 2 * \mathrm{~F} 1)$ data points (at $50 \%$ non-uniform sampling and processed with Iterative Soft Thresholding, IST, reconstruction algorithm) with 48 scans per increment on a Bruker $600 \mathrm{MHz}$ spectrometer equipped with $5 \mathrm{~mm}$ TCI cryoprobe.

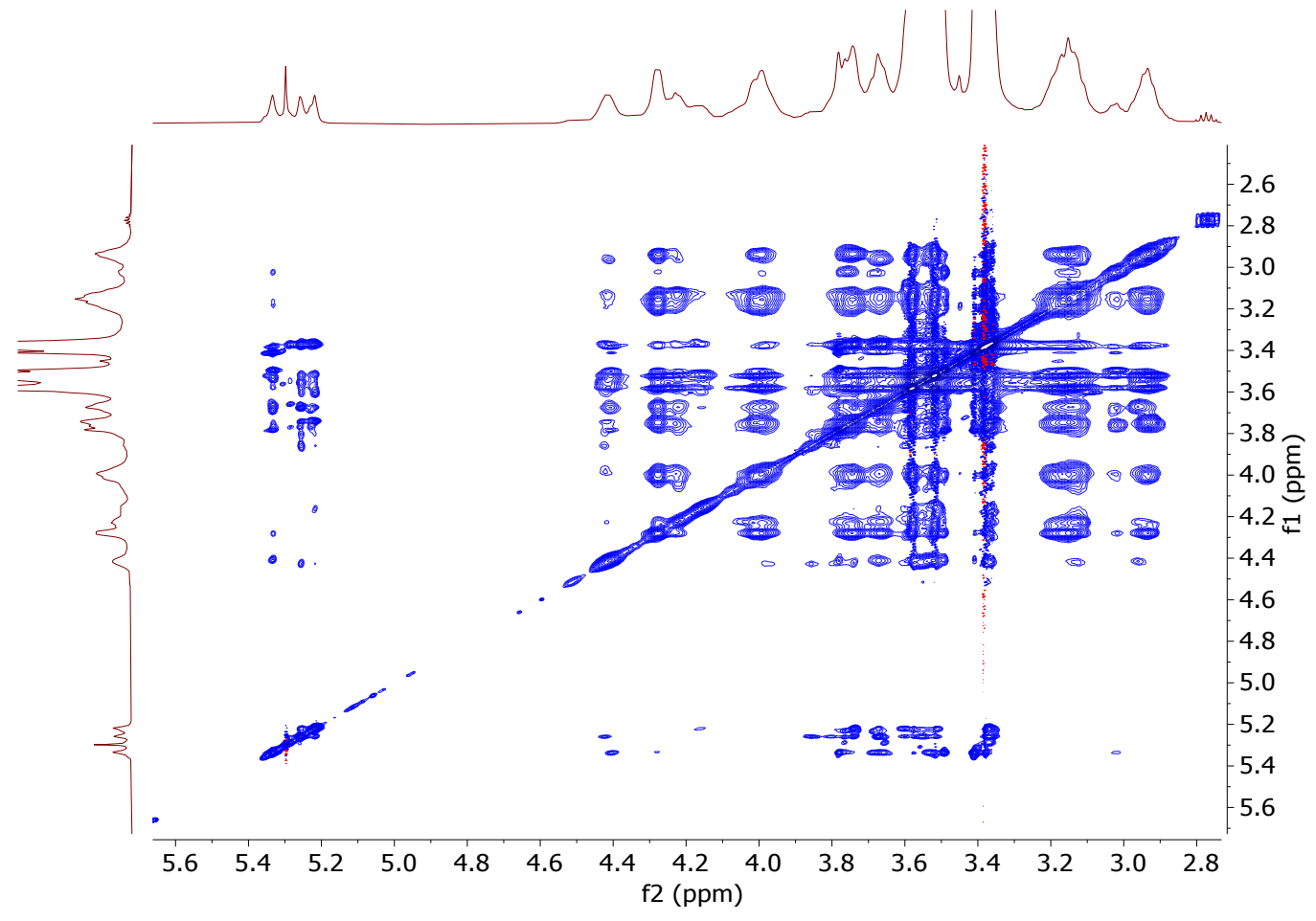

Figure S65: NOESY NMR spectrum of permethylated arabinoxylan polysaccharide standard $(8.7 \mathrm{mg})$, ascquired in $600 \mu \mathrm{L} \mathrm{CDCl}_{3}$ with $1065 * 512(\mathrm{~F} 2 * \mathrm{~F} 1)$ data points with 32 scans per increment on a Bruker $600 \mathrm{MHz}$ spectrometer equipped with $5 \mathrm{~mm}$ TCI cryoprobe. Mixing time $80 \mathrm{~ms}$ 


\section{Glucomannan NMR Data}
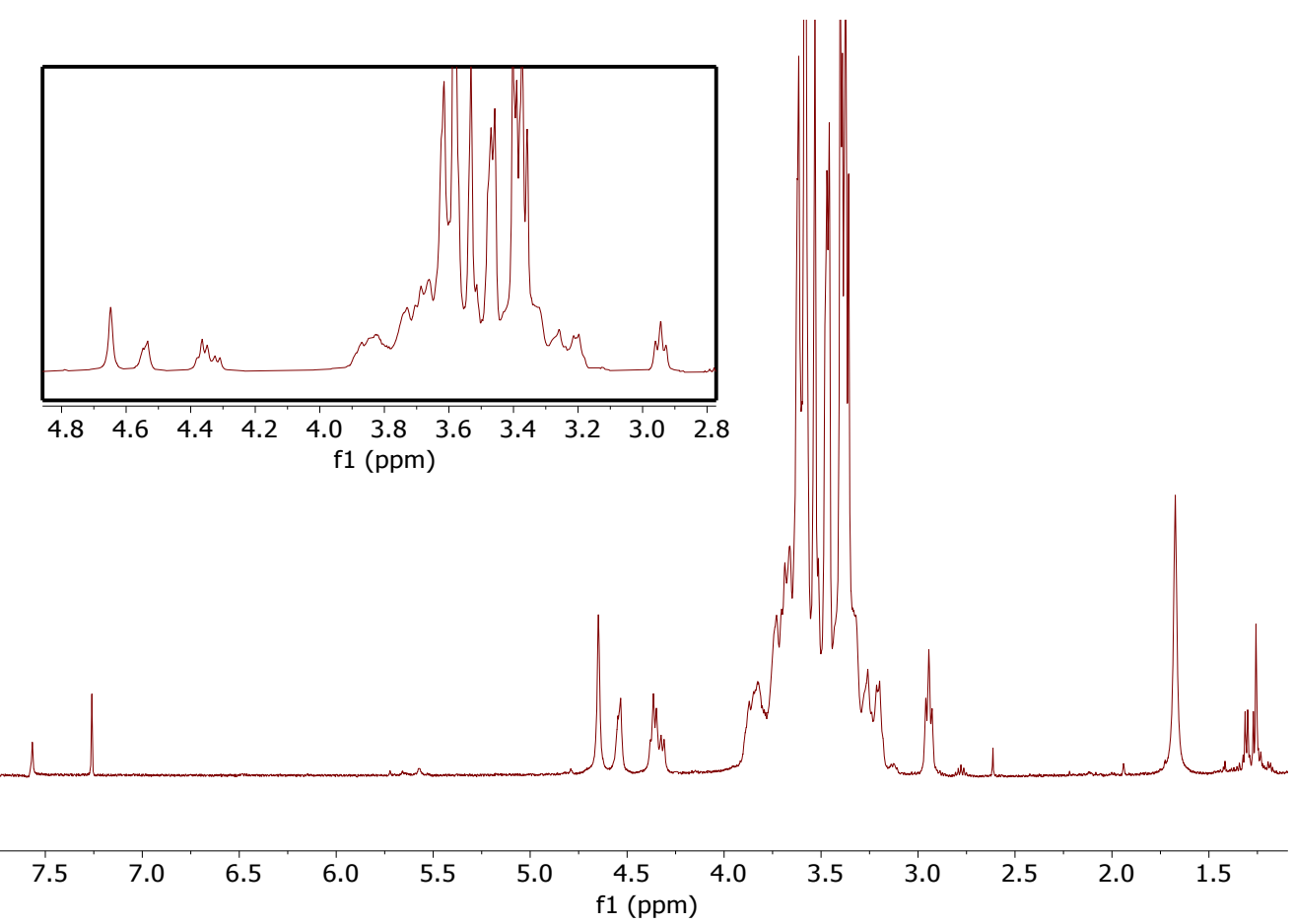

Figure S66: ${ }^{1} \mathrm{H}$ NMR spectrum of permethylated glucomannan polysaccharide standard $(9.7 \mathrm{mg})$, showing an expanded region of $2.80-4.80 \mathrm{ppm}$, acquired in $600 \mu \mathrm{L} \mathrm{CDCl}_{3}$ with 16 scans on a Inova $500 \mathrm{MHz}$ spectrometer equipped with $5 \mathrm{~mm} \mathrm{HNC}$ room temperature probe

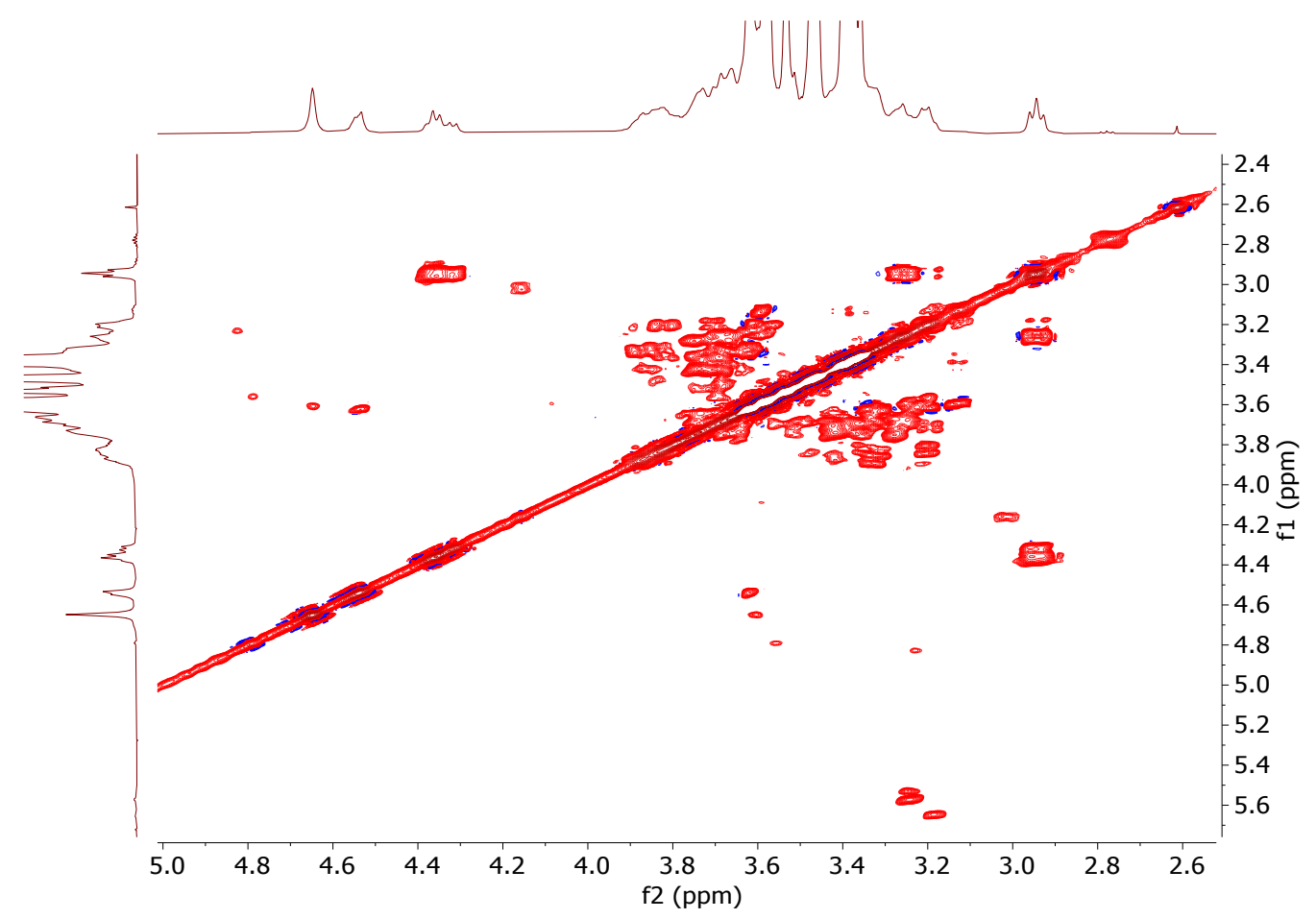

Figure S67: COSY NMR spectrum of permethylated glucomannan polysaccharide standard ( $9.7 \mathrm{mg})$, acquired in $600 \mu \mathrm{L} \mathrm{CDCl}_{3}$ with $1119 * 512(\mathrm{~F} 2 * \mathrm{~F} 1)$ data points with 4 scans per increment on a Inova $500 \mathrm{MHz}$ spectrometer equipped with $5 \mathrm{~mm} \mathrm{HNC}$ room temperature probe. 


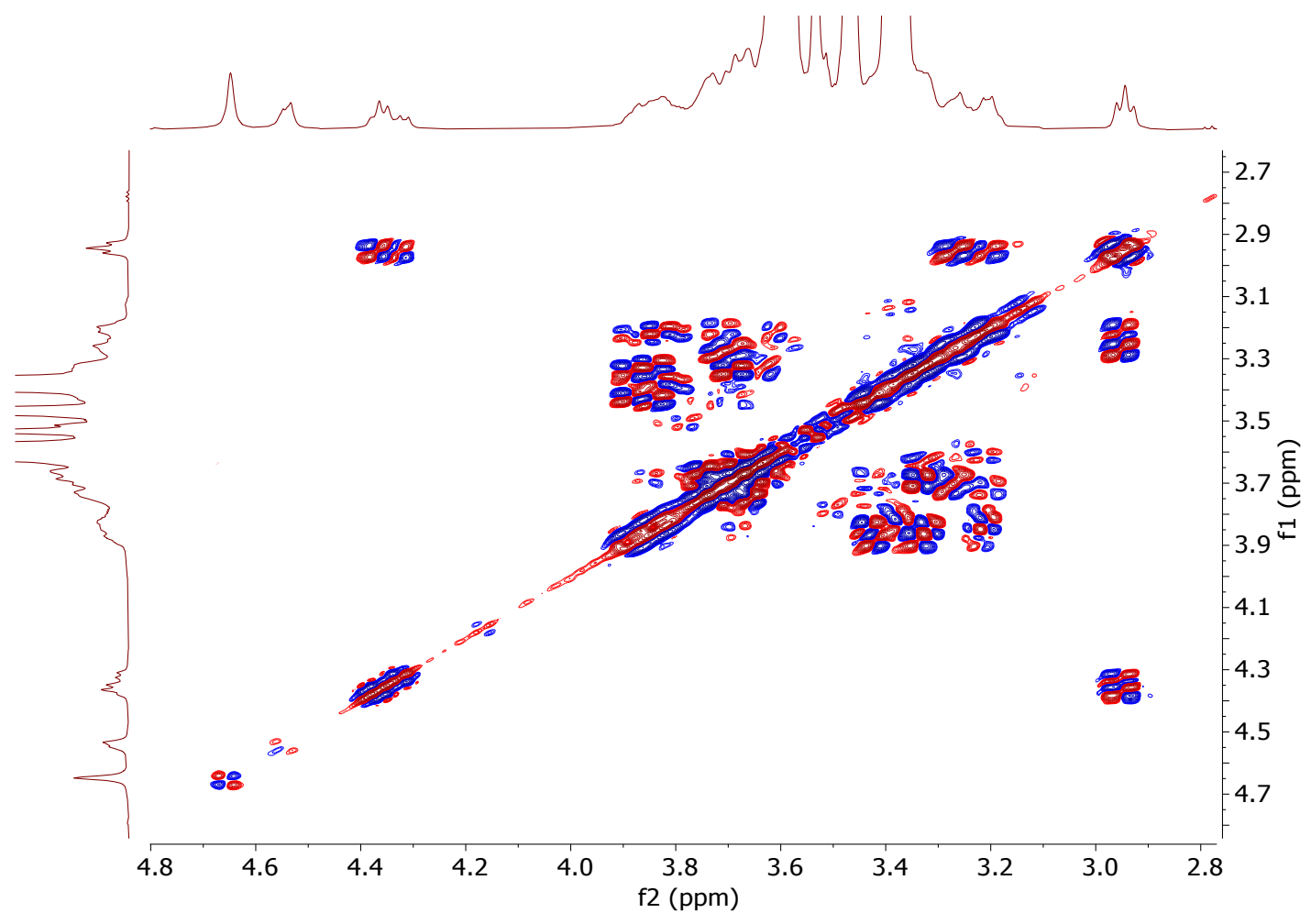

Figure S68: COSY NMR spectrum of permethylated glucomannan polysaccharide standard $(9.7 \mathrm{mg})$, acquired in $600 \mu \mathrm{L} \mathrm{CDCl}_{3}$ with $1119 * 512(\mathrm{~F} 2 * \mathrm{~F} 1)$ data points with 2 scans per increment on a Inova $500 \mathrm{MHz}$ spectrometer equipped with $5 \mathrm{~mm} \mathrm{HNC}$ room temperature probe.

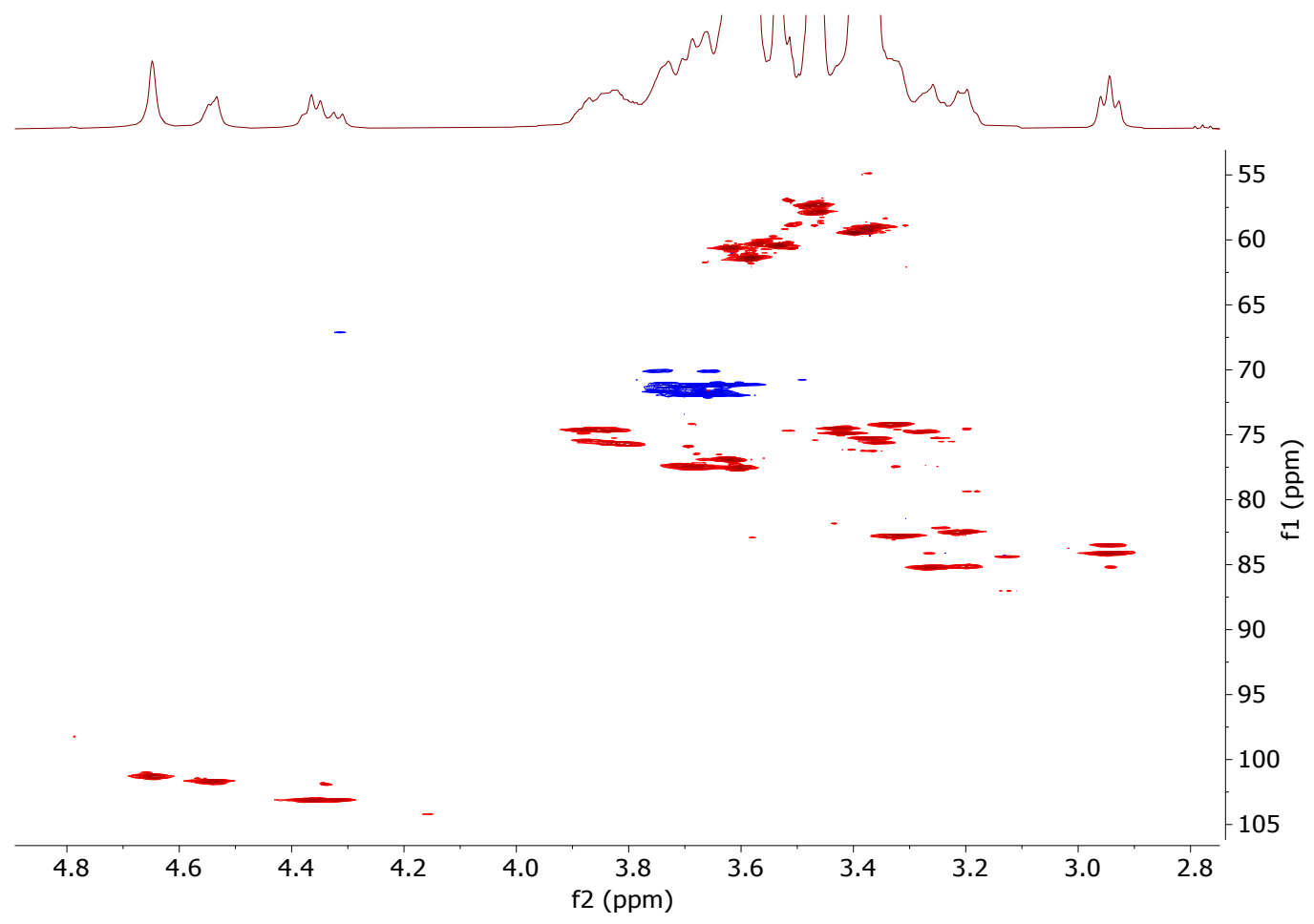

Figure S69: HSQC NMR spectrum of permethylated glucomannan polysaccharide standard (9.7 $\mathrm{mg})$, acquired in $600 \mu \mathrm{L} \mathrm{CDCl}_{3}$ with $819 * 1024(\mathrm{~F} 2 * \mathrm{~F} 1)$ data points with 4 scans per increment on a Bruker $600 \mathrm{MHz}$ spectrometer equipped with $5 \mathrm{~mm}$ TCI cryoprobe. 


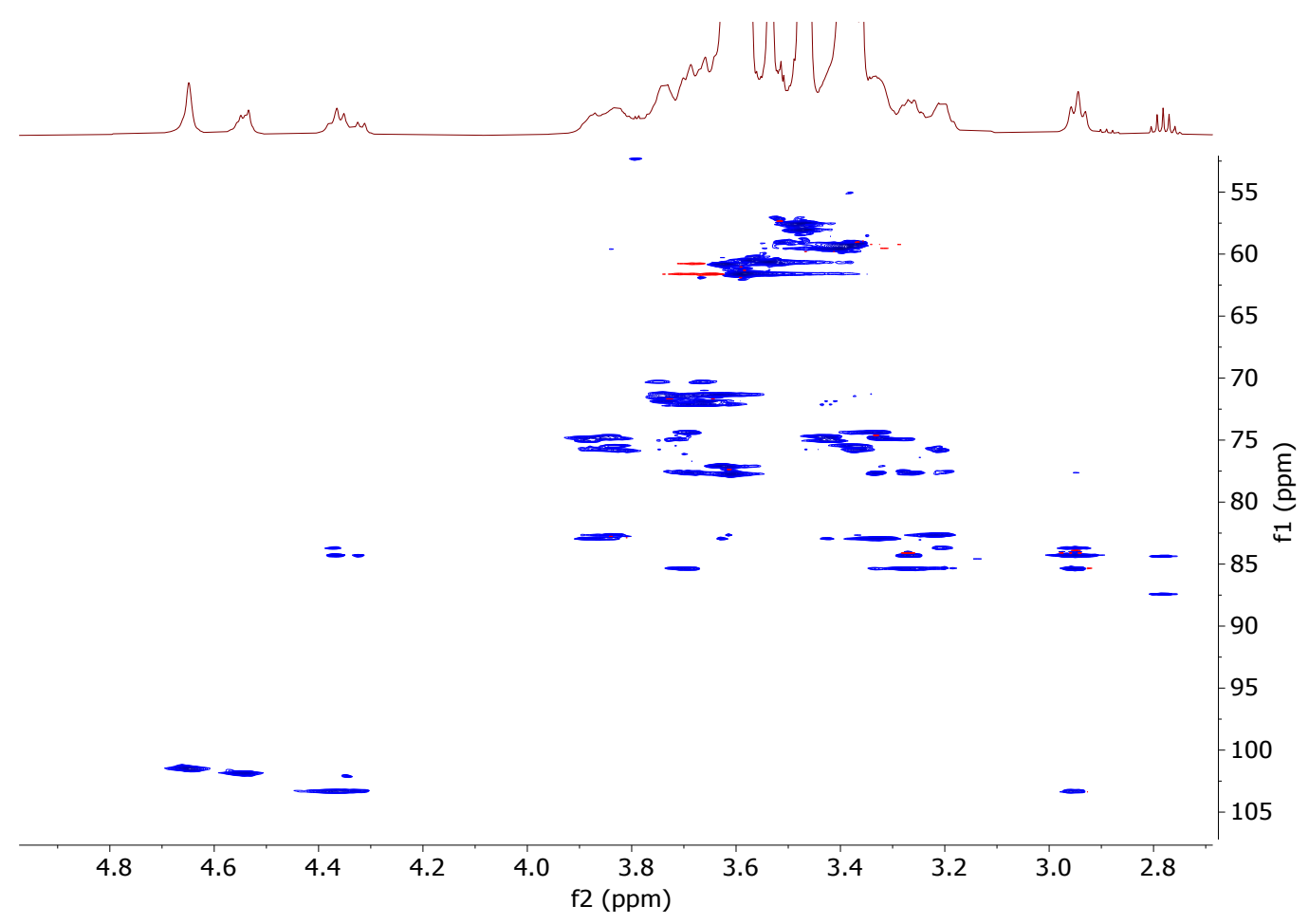

Figure S70: HSQC-TOCSY NMR spectrum of permethylated glucomannan polysaccharide standard $(9.7 \mathrm{mg})$, acquired in $600 \mu \mathrm{L} \mathrm{CDCl}_{3}$ with $1024 * 256(\mathrm{~F} 2 * \mathrm{~F} 1)$ data points with 48 scans per increment on a Bruker $600 \mathrm{MHz}$ spectrometer equipped with $5 \mathrm{~mm}$ TCI cryoprobe.

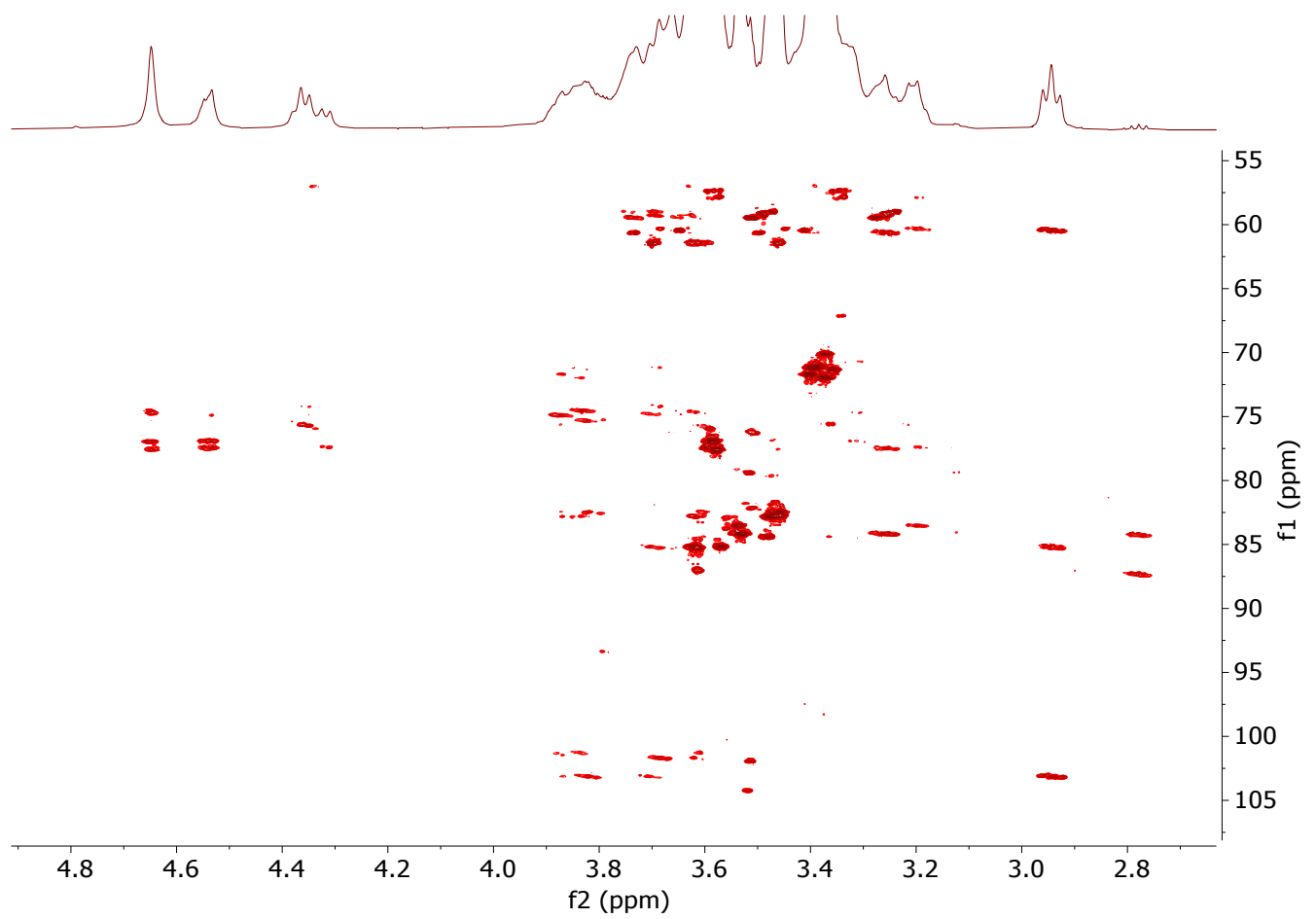

Figure S71: HMBC NMR spectrum of permethylated glucomannan polysaccharide standard $(9.7 \mathrm{mg})$, acquired in $600 \mu \mathrm{L} \mathrm{CDCl}_{3}$ with $1024 * 512(\mathrm{~F} 2 * \mathrm{~F} 1)$ data points with 32 scans per increment on a Bruker $600 \mathrm{MHz}$ spectrometer equipped with $5 \mathrm{~mm}$ TCI cryoprobe. 


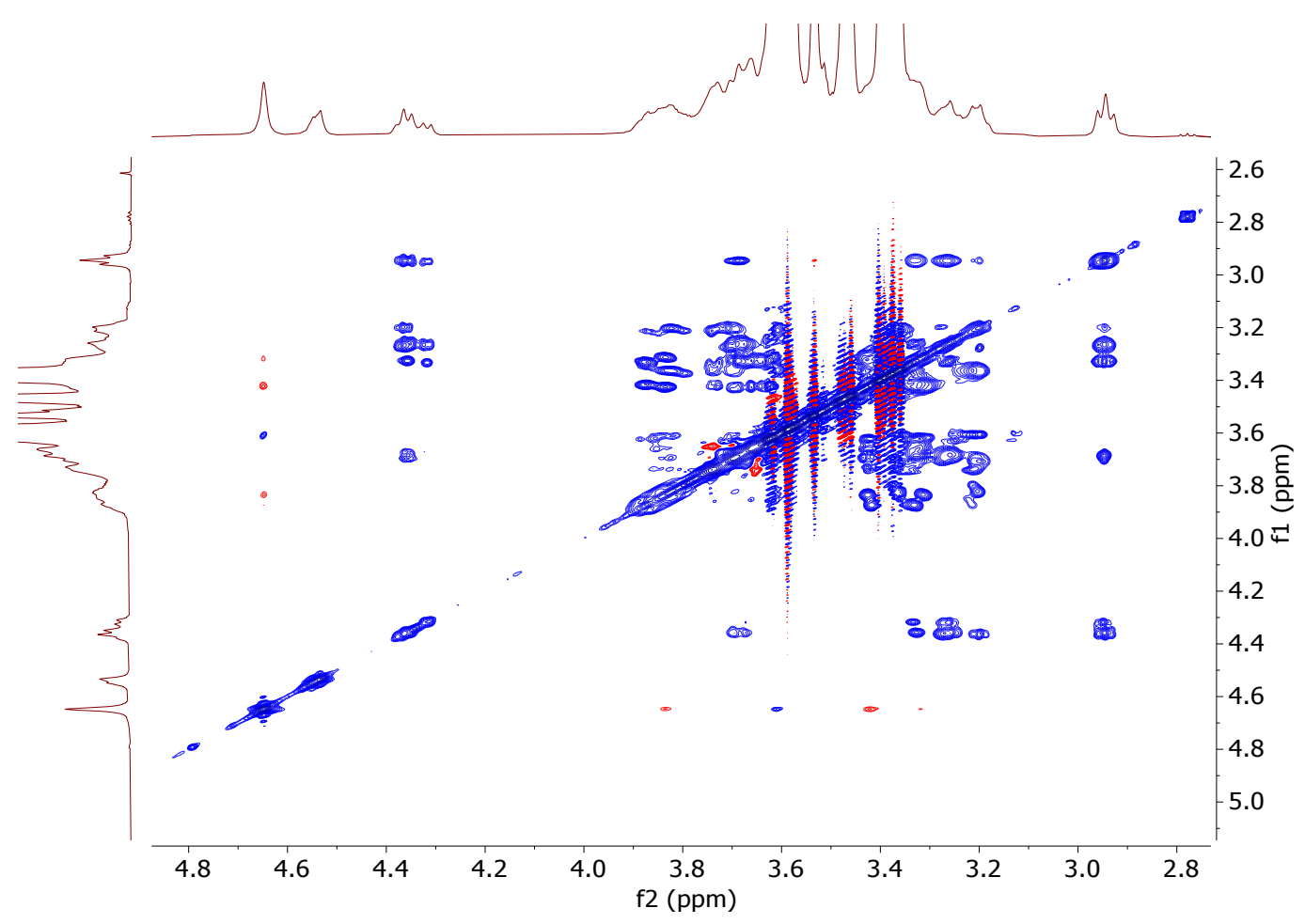

Figure S72: TOCSY NMR spectrum of permethylated glucomannan polysaccharide standard $(9.7 \mathrm{mg})$, acquired in $600 \mu \mathrm{L} \mathrm{CDCl}_{3}$ with $1070 * 384(\mathrm{~F} 2 * \mathrm{~F} 1)$ data points with 24 scans per increment on a VNMRS $600 \mathrm{MHz}$ spectrometer equipped with $5 \mathrm{~mm}$ HNC cryoprobe. $80 \mathrm{~ms}$ spin-lock time.

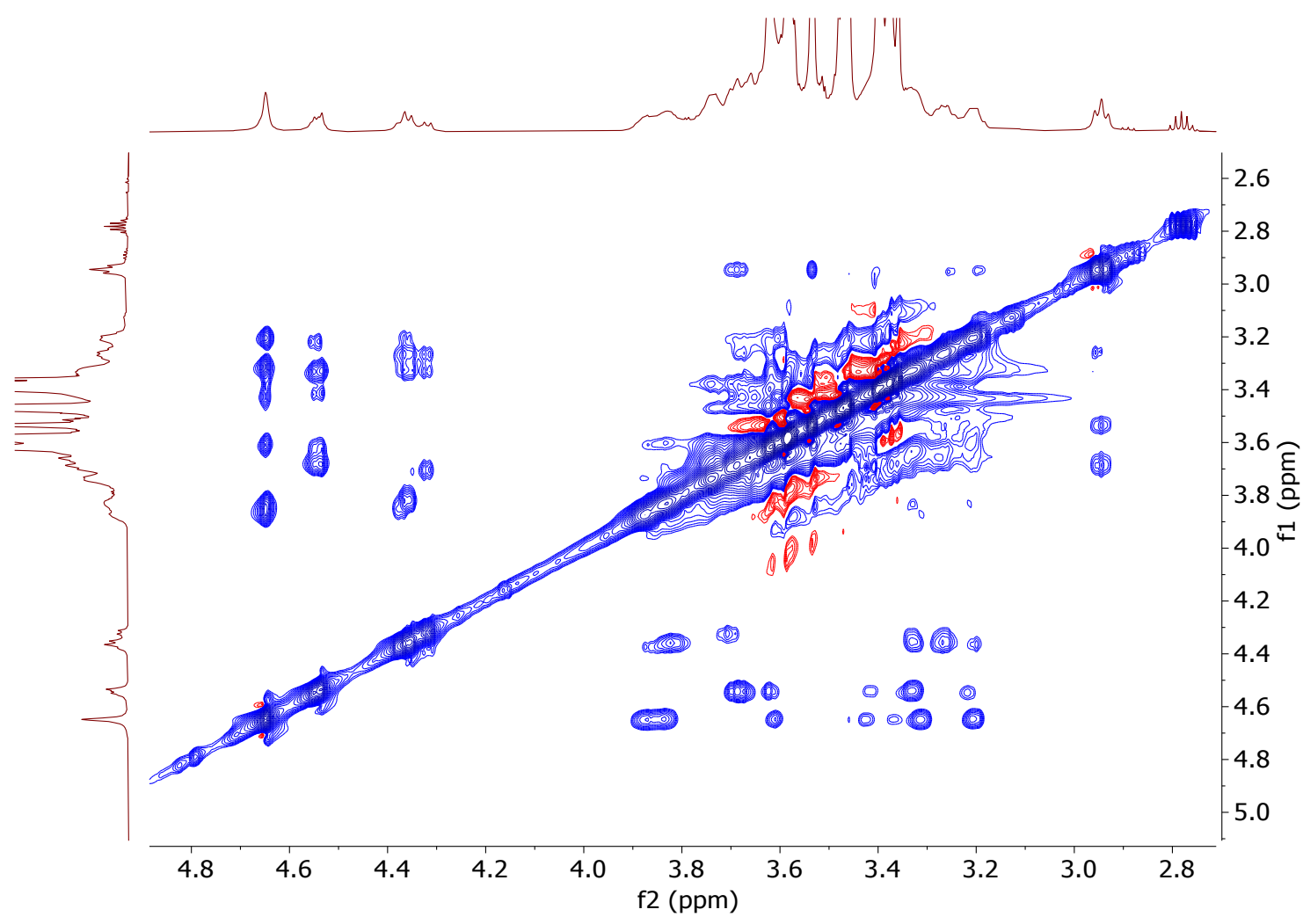

Figure S73: NOESY NMR spectrum of permethylated glucomannan polysaccharide standard $(9.7 \mathrm{mg})$, acquired in $600 \mu \mathrm{L} \mathrm{CDCl}$ with $1024 * 256(\mathrm{~F} 2 * \mathrm{~F} 1)$ data points with 32 scans per increment on a Bruker $600 \mathrm{MHz}$ spectrometer equipped with $5 \mathrm{~mm}$ TCI cryoprobe. 


\section{NMR Acquisition Parameter Optimization}

To optimize NMR acquisition parameters, in particular $J_{\mathrm{CH}}$ coupling evolution time for HSQC and HMBC experiments, $600 \mu \mathrm{L}$ of $\mathrm{CDCl}_{3}$ solution containing $\sim 7.5 \mathrm{mg}$ of permethylated starch and cellulose polysaccharide standards were utilized. This choice of this solution is to factor-in the slight changes in ring residue $J_{\mathrm{CH}}$ coupling that is observed due to changes in anomeric configuration. A series of HSQC experiments were acquired with $135,140,145,150,155,160,165$ and $170 \mathrm{~Hz}^{1} J_{\mathrm{CH}}$ coupling evolution times, whereas a series of $\mathrm{HMBC}$ with ${ }^{\mathrm{n}} J_{\mathrm{CH}}$ coupling evolution times of $4,6,8,10$ and $12 \mathrm{~Hz}$ were acquired. All experiments were acquired with identical parameters, save for the aforementioned $J_{\mathrm{CH}}$ coupling evolution time, respectively, for HSQC and HMBC. To compare peak integrals across different spectra, the scaled absolute peak areas of selected peaks (15 for HSQC and 16 for HMBC) were plotted against $J_{\mathrm{CH}}$ coupling constant values used. For the HSQC experiment, we observed that the best $J_{\mathrm{CH}}$ coupling evolution time compromise would be $145 \mathrm{~Hz}$ - all peaks except for two were at or near peak intensities. In the $\mathrm{HMBC}$ experiment, a $6 \mathrm{~Hz} J_{\mathrm{CH}}$ coupling evolution time would be optimal, as again all peaks were at or near the peak intensities except for two. These results should not be extrapolated to des-methylated polysaccharides, as methylation induces structural changes that impact the $J_{\mathrm{CH}}$ coupling constants.

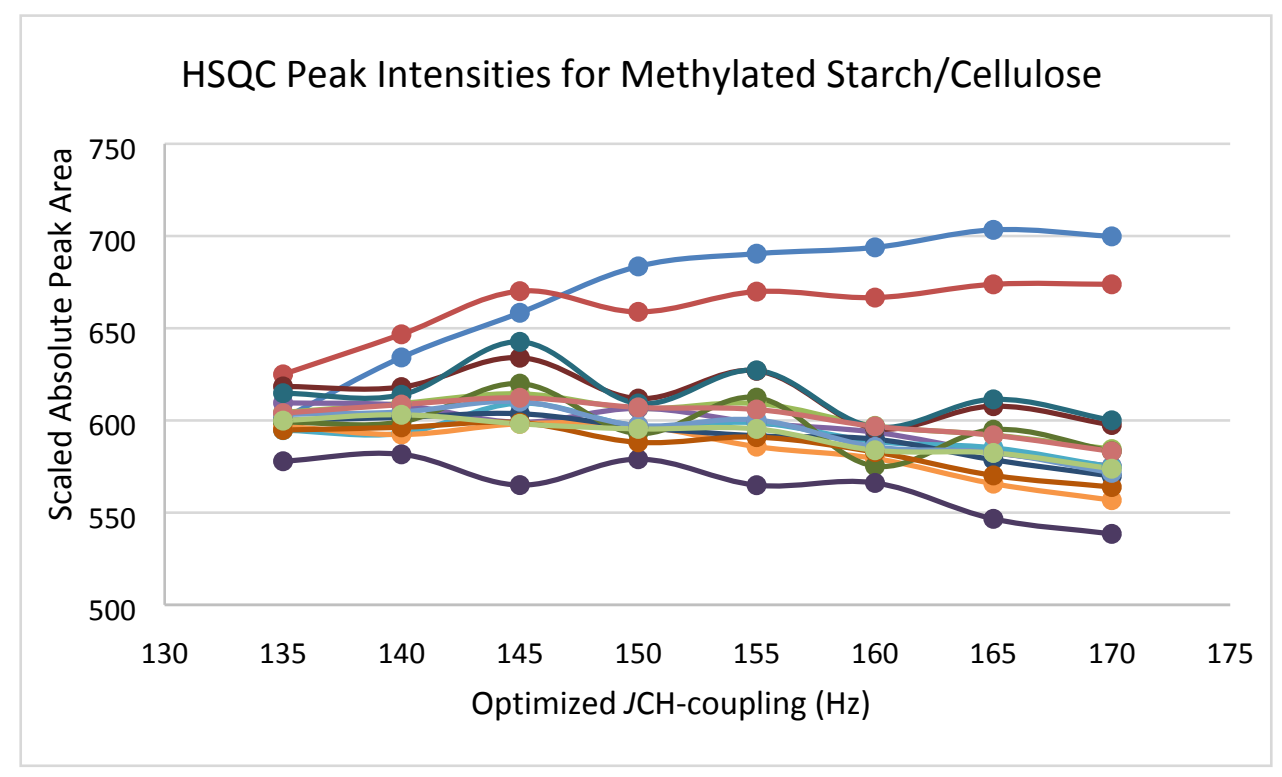

Figure S74: Plots of scaled absolute peak area vs. optimized ${ }^{1} J_{\mathrm{CH}}$ coupling constants used during the evolution time of HSQC multiplicity edited experiments (acquisition parameters; 2 scans, $871 * 384$ increments, $\mathrm{d} 1=1.5 \mathrm{~s}$ ) acquired with a mixture $\sim 7.5 \mathrm{mg}$ starch and cellulose solution in $600 \mu \mathrm{L} \mathrm{CDCl}_{3}$ on a Bruker $600 \mathrm{MHz}$ spectrometer equipped with $5 \mathrm{~mm}$ TCI cryoprobe. The optimal $J_{\mathrm{CH}}$ coupling evolution time for HSQC is taken to be $145 \mathrm{~Hz}$, since most peaks (except two of a total of 15 peaks) showed a maximum or near maximum signal intensity at this value. 


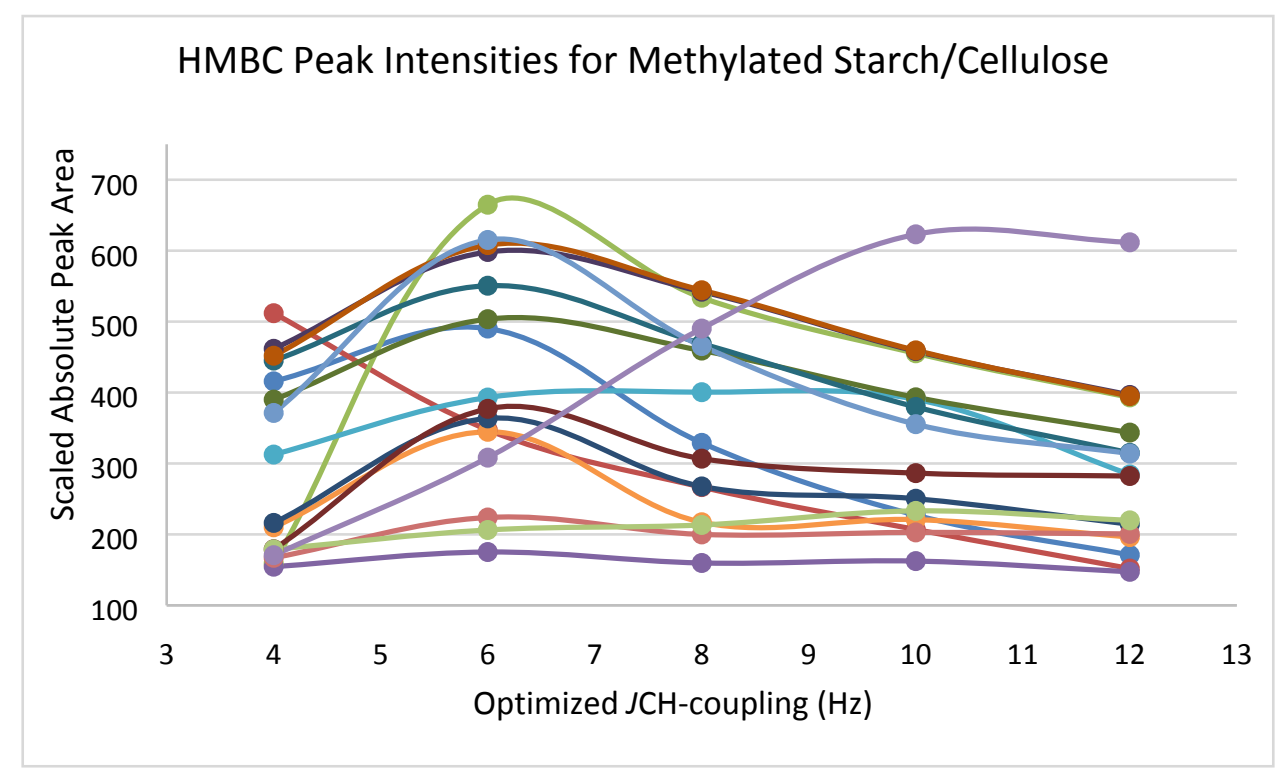

Figure S75: Plots of scaled absolute peak area vs. optimized ${ }^{\mathrm{n}} J_{\mathrm{CH}}$ coupling constants used during the evolution time of HMBC experiments (acquisition parameters; 12 scans, $1536 * 256$ increments, d1 = $1.5 \mathrm{~s}$ ) acquired with a mixture $\sim 7.5 \mathrm{mg}$ starch and cellulose solution in $600 \mu \mathrm{L} \mathrm{CDCl}_{3}$ on a Bruker 600 $\mathrm{MHz}$ spectrometer equipped with $5 \mathrm{~mm}$ TCI cryoprobe. The optimal $J_{\mathrm{CH}}$ coupling evolution time for $\mathrm{HMBC}$ is taken to be $6 \mathrm{~Hz}$, since most peaks (except two of a total of 16 peaks) showed a maximum or near maximum signal intensity at this value.

The mixing time or spin-lock delay for HSQC-TOCSY impact the extent of spin magnetization relay or propagation from the reference spin signal. Our approach in HSQC-TOCSY experiments is to utilize very small spin-lock delays to ensure, as much as possible, that only a single transfer is achieved - that is only adjacent spins will be observed in the HSCQ-TOCSY spectrum. A spin-lock delay of $18 \mathrm{~ms}$ is sufficient (see Figure S76). Longer delays would lead to extended spin magnetization transfer that can further complicate interpretation of an already complex spectrum of plant cell wall polysaccharides (see Figure S77). In addition, longer spin-lock delay leads to loss of magnetization. Absolute peak integrals of the anomeric cross-peaks of starch and cellulose (not shown), indicated an $8 \%$ and $23 \%$ signal loss, respectively, for starch and cellulose, between HSQC-TOCSY spectra acquired with 18 and $60 \mathrm{~ms}$ spinlock delays. 


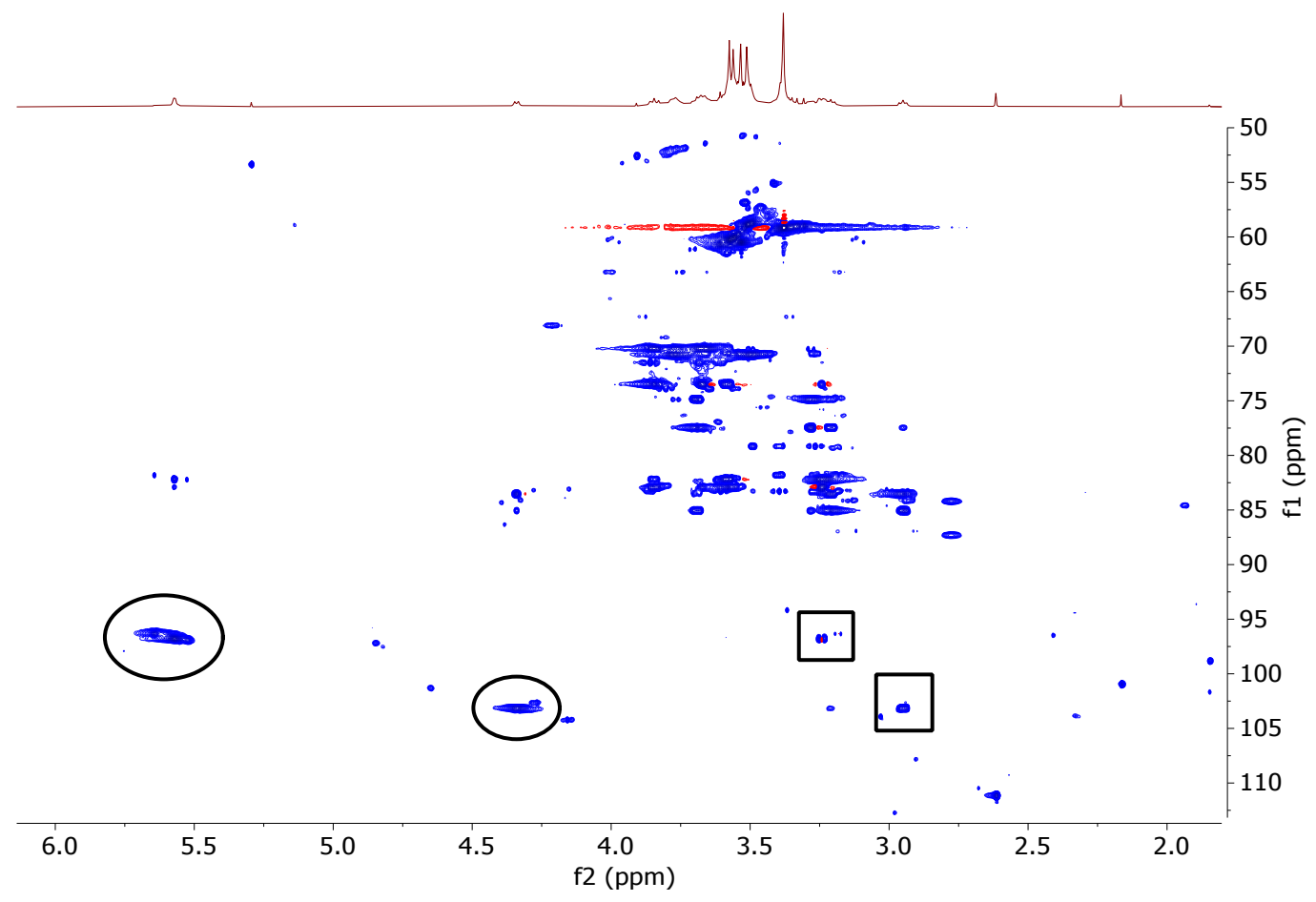

Figure S76: HSQC-TOCSY spectrum of a $600 \mu \mathrm{LCDCl}_{3}$ solution containing $\sim 7.5 \mathrm{mg}$ starch and cellulose mixtures, acquired with 6 scans per increment, $819 * 384(\mathrm{~F} 2 * \mathrm{~F} 1)$ data points, $18 \mathrm{~ms}$ spinlock delay, $1.5 \mathrm{~s}$ recycle delay, on a Bruker $600 \mathrm{MHz}$ spectrometer equipped with $5 \mathrm{~mm}$ TCI cryoprobe. Oval shapes indicate real peaks whereas square shapes indicate TOCSY peaks.

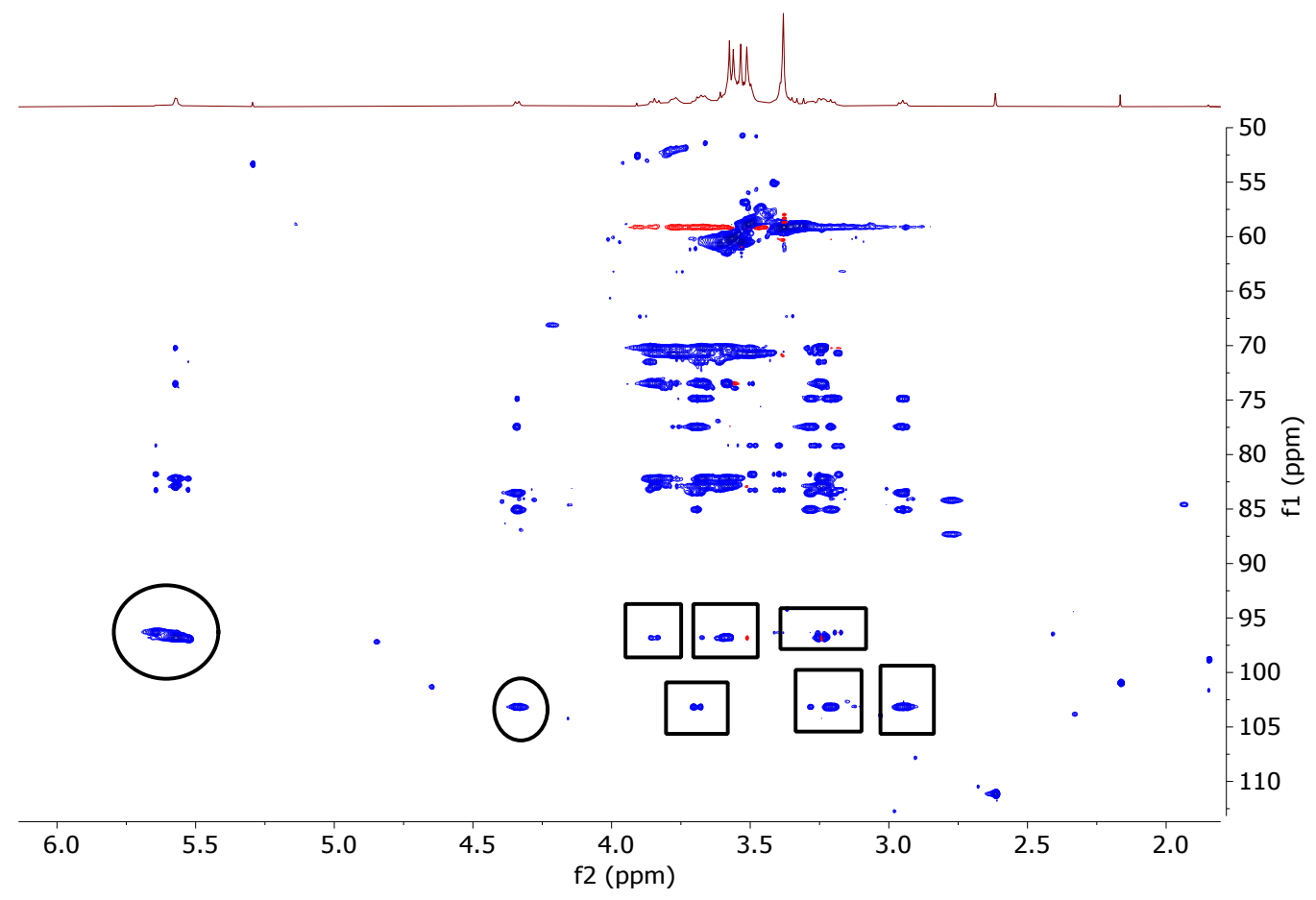

Figure S77: HSQC-TOCSY spectrum of a $600 \mu \mathrm{LCDCl}_{3}$ solution containing $\sim 7.5 \mathrm{mg}$ starch and cellulose mixtures, acquired with 6 scans per increment, $819 * 384(\mathrm{~F} 2 * \mathrm{~F} 1)$ data points, $60 \mathrm{~ms}$ spinlock delay, $1.5 \mathrm{~s}$ recycle delay, on a Bruker $600 \mathrm{MHz}$ spectrometer equipped with $5 \mathrm{~mm}$ TCI cryoprobe. Oval shapes indicate real peaks whereas square shapes indicate TOCSY peaks. 


\section{Switchgrass Glycosyl Linkage Chromatogram}

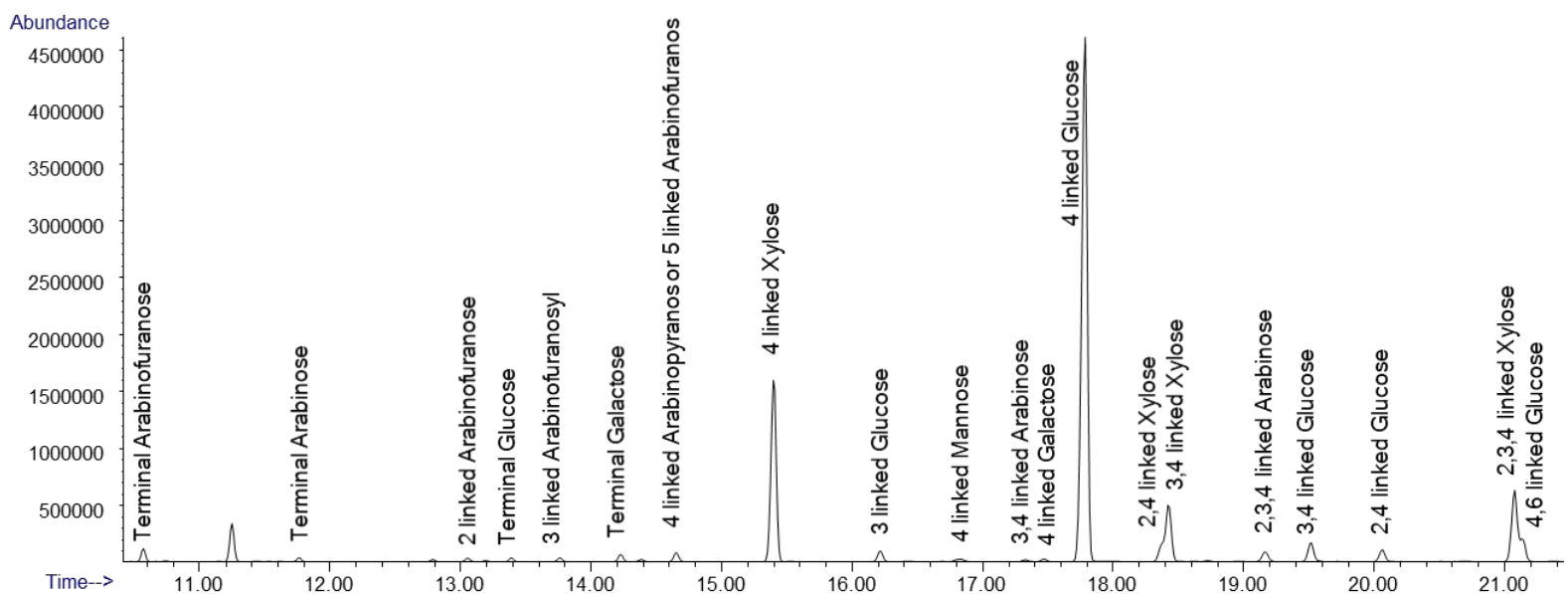

Figure S78: Chromatogram from glycosyl linkage analysis of de-starched, alcohol insoluble switchgrass sample.

\section{Poplar Glycosyl Linkage Chromatogram}

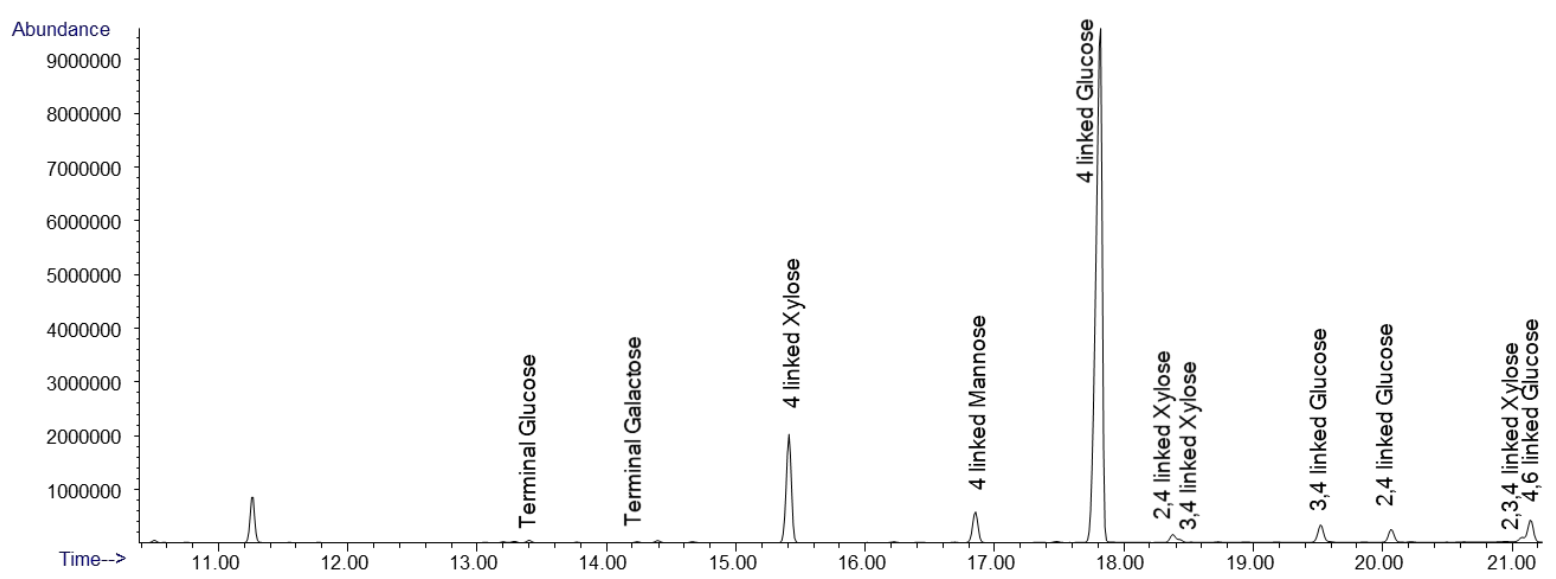

Figure S79: Chromatogram from glycosyl linkage analysis of de-starched, alcohol insoluble poplar sample. 


\section{References}

1 S. D. Mansfield, H. Kim, F. Lu and J. Ralph, Nat. Protoc., 2012, 7, 1579-1589.

2 K. R. Anumula and P. B. Taylor, Anal. Biochem., 1992, 203, 101-108.

3 I. Ciucanu and F. Kerek, Carbohydr. Res., 1984, 131, 209-217.

4 F. A. Pettolino, C. Walsh, G. B. Fincher and A. Bacic, Nat. Protoc., 2012, 7, 1590-1607.

5 C. Heiss, J. Stacey Klutts, Z. Wang, T. L. Doering and P. Azadi, Carbohydr. Res., 2009, 344, 915-920.

6 F. Dourado, S. Cardoso, A. Silva, F. Gama and M. Coimbra, Carbohydr. Polym., 2006, 66, $27-33$.

$7 \quad$ M. Lundborg and G. Widmalm, Anal. Chem., 2011, 83, 1514-1517.

$8 \quad$ J.-Y. Yin, H.-X. Lin, S.-P. Nie, S. W. Cui and M.-Y. Xie, Carbohydr. Polym., 2012, 88, 13951401.

9 A. Höije, C. Sandström, J. P. Roubroeks, R. Andersson, S. Gohil and P. Gatenholm, Carbohydr. Res., 2006, 341, 2959-2966.

10 R. A. Hoffmann, J. P. Kamerling and J. F. G. Vliegenthart, Carbohydr. Res., 1992, 226, 303311. 